\title{
Clincial Translational Science Research of the Functional Role of Large Conductance Potassium Channels in Selective Destruction of Triple Negative Breast Cancer Cells
}

\author{
Gina Sizemore \\ gls0016@mix.wvu.edu
}

Follow this and additional works at: https://researchrepository.wvu.edu/etd

Part of the Chemical and Pharmacologic Phenomena Commons, Genetic Phenomena Commons, Medical Biophysics Commons, Medical Genetics Commons, and the Translational Medical Research Commons

\section{Recommended Citation}

Sizemore, Gina, "Clincial Translational Science Research of the Functional Role of Large Conductance Potassium Channels in Selective Destruction of Triple Negative Breast Cancer Cells" (2020). Graduate Theses, Dissertations, and Problem Reports. 7731.

https://researchrepository.wvu.edu/etd/7731

This Dissertation is protected by copyright and/or related rights. It has been brought to you by the The Research Repository @ WVU with permission from the rights-holder(s). You are free to use this Dissertation in any way that is permitted by the copyright and related rights legislation that applies to your use. For other uses you must obtain permission from the rights-holder(s) directly, unless additional rights are indicated by a Creative Commons license in the record and/ or on the work itself. This Dissertation has been accepted for inclusion in WVU Graduate Theses, Dissertations, and Problem Reports collection by an authorized administrator of The Research Repository @ WVU.

For more information, please contact researchrepository@mail.wvu.edu. 
CLINICAL TRANSLATIONAL SCIENCE RESEARCH OF THE FUNCTIONAL ROLE OF LARGE CONDUCTANCE POTASSIUM CHANNELS IN SELECTIVE DESTRUCTION OF TRIPLE NEGATIVE BREAST CANCER CELLS

Gina Sizemore

Follow this and additional works at: https://researchrepository.wvu.edu/etd

Part of the Chemical and Pharmacologic Phenomena Commons, Genetic Phenomena Commons, Medical Biophysics Commons, Medical Genetics Commons, and the Translational Medical Research Commons 


\title{
CLINICAL TRANSLATIONAL SCIENCE RESEARCH \\ OF
}

THE FUNCTIONAL ROLE OF LARGE CONDUCTANCE POTASSIUM CHANNELS IN SELECTIVE DESTRUCTION OF TRIPLE NEGATIVE BREAST CANCER CELLS

\section{Gina Sizemore}

Dissertation submitted to the School of Medicine at West Virginia University

In Partial Fulfillment of the Requirements for the Doctor of Philosophy Degree in Clinical Translational Science/Ion Channel Electrophysiology

\author{
Julie Lockman, PhD., Chair \\ Werner Geldenhuys, PhD. \\ Peter Guida, PhD. \\ Malcolm Mattes, PhD. \\ Hangang Yu, PhD. \\ Department of Clinical Translational Science
}

Morgantown, West Virginia

2020

Keywords: Ion Channels, TNBC, Electrophysiology, Applied Physics, Potassium Channels, Breast Cancer, Biophysics 


\section{ABSTRACT \\ Clinical Science Research of the Functional Role of Large Conductance Potassium Channels in Selective Destruction of Triple Negative Breast Cancer Cells.}

\section{Gina Sizemore}

The preliminary background that puts this research into context is threefold; it is the aggressive nature of triple negative breast cancer (TNBC), the complexity surrounding its pathology, and the significant lack of targeted treatment for this disease. To clarify the focus of my research, I have concentrated on identifying a targeted treatment for TNBC. In the process, I have identified cycles of reciprocity between treatment, clinical diagnosis, staging, and pathology that will be addressed in smaller papers. However, the weight of this work is in the discovery of a novel target for triple negative breast cancer. The value of this research is evidenced by the significant discovery of a novel ion channel target that demonstrates selective, targeted destruction of TNBC without damage to healthy tissue. The specific research aims and objectives are to first, characterize the big conductance calcium and voltage gated potassium channel (BK) in TNBC to investigate the apparent contradiction of a depolarized (more positive) resting membrane potential within the context of a membrane with overexpression of channels that cause hyperpolarization (more negative) in the membrane potential. Secondly, examine hyperpolarization of TNBC cells as the mechanism of cell death by severe efflux of potassium

(K) from the TNBC cell via these overexpressed BK channels. Thirdly, establish the first steps for human translational research of this discovery with evaluation of human TNBC tumor biopsies for similar overexpression of this BK channel. In summary, the objectives are to fill the knowledge gap by demonstrating BK channels are not functional (active) in TNBC [which explains why we can have a positive (depolarized) cell that should be more negative (hyperpolarized) due to the overexpression of the BK channel but is not]. Secondly, demonstrate that this overexpression of non-functioning (not-active) channels can be exploited by activating those channels and hyperpolarizing the cells with efflux of their potassium from the cell as a mechanism for selective (not impacting healthy cells), targeted treatment for TNBC. Finally, translating that to a first step evaluation of human TNBC from tumor biopsies.

The additional supportive papers in the scope of this work are in chapter two and chapter four surrounding the main work in chapter three. The supportive work in chapter two exists to identify the opportunity for electrophysiology as a tool for translating basic science discovery to clinical application and is listed as reference [1]. The supportive work in chapter four exposes an epidemiologic discovery of a translational gap in the clinical diagnosis of triple negative breast cancer (manuscript under review) where ion channels can impact. 
This dissertation interpolates information from the afore mentioned papers by the author. As described, chapter two references material from reference [1] and was authored as follows: Gina Sizemore1, Brandon Lucke-Wold2, Charles Rosen2, James W. Simpkins3, Sanjay Bhatia2, and Dandan Sun4. Chapter three references material from the main body of work and was authored as follows: Gina Sizemore1, Sarah McLaughlin2, Mackenzie Newman3, Kathleen Brundage4, Amanda Ammer2, Karen Martin2, Elena Pugacheva5, James Coad6, Malcolm D. Mattes7, Han-Gang Yu3. Finally, chapter four supports the research with the transition to epidemiology and references material authored as follows: Gina Sizemore1, Toni Rudisill2.

In summary, this composition weaves a thread across the clinical translational science field intertwining chapter two (paper 1) a methods paper of ion channels and the electrophysiology tool, with chapter three (paper 2) the novel discovery of an ion channel target, and the epidemiologic opportunity for ion channel translation in chapter four (paper 3). 


\section{TABLE OF CONTENTS}

Table of Contents iv

List of Text Diagrams ..............................................................

Acknowledgements................................................................ viii

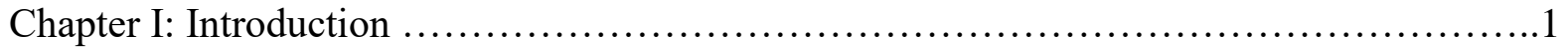

Chapter II: [Manuscript 1] Temporal Lobe Epilepsy, Stroke, and Traumatic Brain Injury: Mechanisms of Hyperpolarized, Depolarized, and Flow-Through Ion Channels Utilized as TriCoordinate Biomarkers of Electrophysiologic Dysfunction....

Summary \& Transition Paragraph

Chapter III: [Manuscript 2] Opening Large-Conductance Potassium Channels Selectively Induced Cell Death of Triple Negative Breast Cancer.

Summary \& Transition Paragraph

Chapter IV: [Manuscript 3] Triple Negative Breast Cancer: Exponential Tumor Grade Increase with Age of Diagnosis

Summary \& Transition Paragraph

Chapter V: Discussion and Translational Significance

Chapter VI: Methods/Techniques of Research.

Chapter VII: Literature Review .66

A. Structure of High-Conductance Calcium-Activated Potassium Channels (BK K $\left.\mathrm{c}_{\mathrm{ca}}\right)$

1. Alpha Unit coded for by KNCMA1

a. The $\alpha-1$ with $\mathrm{N}$-Terminus MANG

b. The $\alpha-1$ with $\mathrm{N}$-Terminus MSSN

c. The $\alpha-1$ with N-Terminus MDAL

d. The $\alpha-1$ components

1. Transmembrane component (7 segments $\{\mathrm{S} 0-\mathrm{S} 6\})$

a. S0 note extra hydrophobic segment at $\mathrm{NH}_{2}$ terminus

b. S1 VSDomain

c. S2 VSDomain

d. S3 VSDomain

e. S4 VSDomain

f. S5 Pore Domain

g. S6 Pore Domain

2. C-terminus

a. four hydrophobic segments 
b. alternative splicing sites

c. stretch of negatively charged amino acids (aspartates) called $\mathrm{Ca}^{2+}$ bowl

d. regulator conductance of potassium (RCK1) Each $\alpha-1$ contributes 2 RCK

e. regulator conductance of potassium (RCK2)

f. gating ring forms from the $8 \mathrm{RCK}$; Each of the (4) $\alpha-1$ contributes 2 RCK

\section{Beta Unit}
a. Beta subunit modifier
b. Beta subunit 1-4
c. Beta subunit controversy

3. Pore Unit

a. Part of the Alpha Unit

b. Homotetramer - the identical four subunit complex of the BK channel demonstrates the association (not covalent bonding) between the subunits.

c. contribute region where TEA, IBX, and Charbydtoxin work

d. external loop (turret) between $\mathrm{S} 5$ and pore helix has more residues than other $\mathrm{K}^{+}$ 4. Has the crystal structure been solved?

a. clarify location of $\mathrm{S} 0$ in respect to the Voltage sensor domain of $\alpha-1$ unit

B. Function of High-Conductance Calcium-Activated Potassium Channels (BK $\mathrm{K}_{\mathrm{ca}}$ )

1. Open Probability with Depolarization
a. cell proliferation
1. depolarized $\mathrm{Vm}$ favors proliferation
b. cell differentiation
c. cell migration
d. cell cycle progression

2. Open Probability with Intracellular Calcium

a. $\mathrm{Ca}^{2+}$ Calcium influx impact excitable cell

1. resting membrane potential excitable cells

2. action potential excitable cells

b. $\mathrm{Ca}^{2+}$ Calcium influx impact non-excitable TNBC

1. resting membrane potential non-excitable cell

2. homeostasis shift non-excitable cells

\section{Goldman-Hodgkin-Katz equation}
a. $\mathrm{Na}^{1+}$ Sodium
b. $\mathrm{K}^{1+}$ Potassium - significant role in $\mathrm{Vm}$ regulation in cancer
c. $\mathrm{Cl}^{1-}$ Chloride

C. Anatomy and Physiology of triple negative breast cancer (TNBC) MDA MB 231 Cell

1. Origin

2. Morphology

3. Membrane potential 
D. TNBC The Disease

1. Breast cancer types

2. TNBC subtypes

3. Survival

4. Metastatic risk

5. Recurrence

E. TNBC Treatment Options

1. Resection

2. Radiation

3. Chemotherapy

F. Breast Cancer Testing (CS site specific Co-factors from WV cancer registry \& SEER)

1. Estrogen Receptor (ER) Assay

2. Progesterone Receptor (PR) Assay

3. Number of Positive Ipsilateral Level I-II Axillary Lymph Nodes

4. Immunohistochemistry (IHC) of Regional Lymph Nodes

5. Molecular (MOL) Studies of Regional Lymph Nodes

6. Size of Tumor-Invasive Component

7. Nottingham or Bloom-Richardson (BR) Score/Grade

8. HER2: Immunohistochemistry (IHC) Lab Value

9. HER2: Immunohistochemistry (IHC) Test Interpretation

10. HER2: Fluorescence In Situ Hybridization (FISH) Lab Value

11. HER2: Fluorescence In Situ Hybridization (FISH) Test Interpretation

12. HER2: Chromogenic In Situ Hybridization (CISH) Lab Value

13. HER2: Chromogenic In Situ Hybridization (CISH) Test Interpretation

14. HER2: Result of Other or Unknown Test

15. HER2: Summary Result of Testing

16. Combinations of ER, PR, and HER2 Results

17. Circulating Tumor Cells (CTC) and Method of Detection

18. Disseminated Tumor Cells (DTC) and Method of Detection

19. Assessment of Positive Ipsilateral Axillary Lymph Nodes

20. Assessment of Positive Distant Metastases

21. Response to Neoadjuvant Therapy

22. Multigene Signature Method

23. Multigene signature test

24. Paget Disease

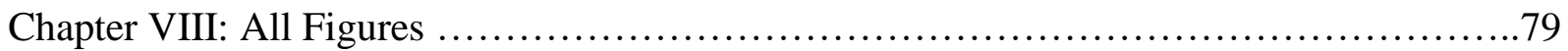

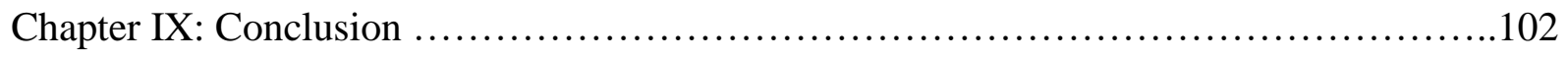




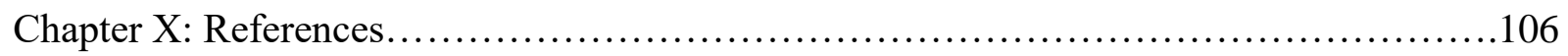

Appendix A: Clinical Translational Science Definition and Purpose $\quad$...................111

Appendix B: Additional References............................................ 113

[Manuscript 1] https://www.ncbi.nlm.nih.gov/pmc/articles/PMC6018002/

Sizemore, G. et al. Temporal Lobe Epilepsy, Stroke, and Traumatic Brain Injury: Mechanisms of Hyperpolarized, Depolarized, and Flow-Through Ion Channels Utilized as Tri-Coordinate Biomarkers of Electrophysiologic Dysfunction. OBM Neurobiol. 2, (2018).

[Manuscript 2] https://bmccancer.biomedcentral.com/articles/10.1186/s12885-020-07071-1

Sizemore, G. et al. Opening large-conductance potassium channels selectively induced cell death of triple-negative breast cancer. BMC Cancer 20, 595 (2020).

[Manuscript 3] In Submission at time of dissertation

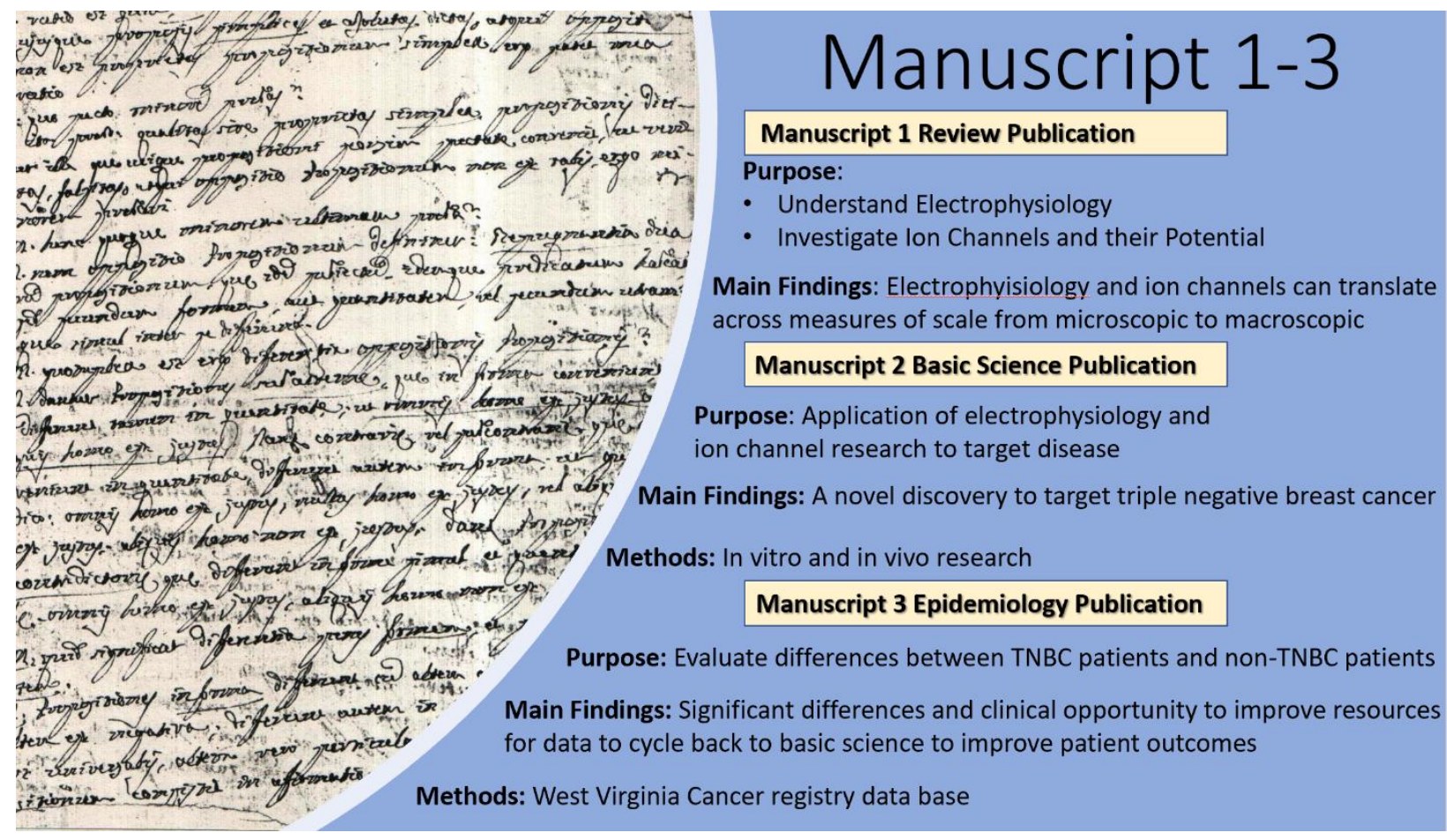




\section{LIST OF TEXT DIAGRAMS}

Diagram 1: BK Channels Forced Open/Activated with BK Channel Opener................61

Diagram 2: BK Channel Opener Halts Cell Cycle at G2 $\ldots \ldots \ldots \ldots \ldots \ldots \ldots \ldots \ldots \ldots \ldots \ldots \ldots . \ldots \ldots$

Diagram 3: How cells mimic electrical circuits.................................... 71

Diagram 4: Understanding voltage and membrane potential.........................72

Diagram 5: Membrane Potential: from threshold to hyperpolarization...................73

Diagram 6: Resting membrane potential TNBC vs. normal cells.........................74

\section{ACKNOWLEDGEMENTS}

Dr. James Simpkins, Department of Neuroscience WVU, he believed in me and made me a believer in Goethe's quote "The moment one definitely commits oneself, then providence moves too. Whatever you think you can do, or believe you can do, begin it. Action has magic, power, and grace."

Dr. Hangang Yu, Department of Physiology and Pharmacology WVU, his love of discovery and good science inspired me to improve my detail skills to reap the rewards of scientific discovery.

Dr. Julie Lockman, Department of Physiology and Pharmacology WVU, her leadership and compassion guided me in some of the toughest times of my program.

Dr. Werner Geldenhuys, Department of Neuroscience WVU, his friendly openness, problem solving quickness, and genuine love of students made each learning experience memorable.

Dr. Peter Guida, from the Brookhaven National lab in New York, his empowerment of students and innovative thinking encouraged me to challenge myself and achieve more than I ever dreamed.

Dr. Bernard Schreurs, Rockefellar Neuroscience Institute WVU, his willingness to give of his time to mentor and guide me amidst his busy schedule reminded me that good teachers teach by example.

My Family, I specifically want to thank Penny for her significant sacrifice and support these four years. You are the incredible wind beneath my wings. I am grateful for my parents and siblings and want to acknowledge each of them. One of the greatest gifts is a family who teaches you failure is part of success. From childhood we were encouraged to learn, explore, fail, and succeed with the constant of love. 


\section{CHAPTER ONE}

\section{Introduction}

The preliminary background that puts this research into context is threefold; it is the aggressive nature of triple negative breast cancer (TNBC), the complexity surrounding its pathology, and the significant lack of targeted treatment for this disease. To clarify the focus of my research, I have concentrated on identifying a targeted treatment for TNBC. In the process, I have identified cycles of reciprocity between treatment, clinical diagnosis, staging, and pathology that will be addressed in smaller papers. However, the weight of this work is in the discovery of a novel target for triple negative breast cancer. The value of this research is evidenced by the significant discovery of a novel ion channel target that demonstrates selective, targeted destruction of TNBC without damage to healthy tissue. The specific research aims and objectives are to first, characterize the big conductance calcium and voltage gated potassium channel (BK) in TNBC to investigate the apparent contradiction of a depolarized (more positive) resting membrane potential within the context of a membrane with overexpression of channels that cause hyperpolarization (more negative) in the membrane potential. Secondly, examine hyperpolarization of TNBC cells as the mechanism of cell death by severe efflux of potassium (K) from the TNBC cell via these overexpressed BK channels. Thirdly, establish the first steps for human translational research of this discovery with evaluation of human TNBC tumor biopsies for similar overexpression of this BK channel. In summary, the objectives are to fill the knowledge gap by demonstrating BK channels are not functional (active) in TNBC [which explains why we can have a positive (depolarized) cell that should be more negative (hyperpolarized) due to the overexpression of the BK channel but is not]. Secondly, demonstrate that this overexpression of non-functioning (not-active) channels can be exploited by activating 
those channels and hyperpolarizing the cells with efflux of their potassium from the cell as a mechanism for selective (not impacting healthy cells), targeted treatment for TNBC. Finally, translating that to a first step evaluation of human TNBC from tumor biopsies.

The additional supportive papers in the scope of this work are in chapter two and chapter four surrounding the main work in chapter three. The supportive work in chapter two exists to identify the opportunity for electrophysiology as a tool for translating basic science discovery to clinical application and is listed as reference [1]. The supportive work in chapter four exposes an epidemiologic discovery of a translational gap in the clinical diagnosis of triple negative breast cancer (manuscript under review) where ion channels can impact. This dissertation interpolates information from the afore mentioned papers by the author. As described, chapter two references material from reference [1] and was authored as follows: Gina Sizemore ${ }^{1}$, Brandon LuckeWold $^{2}$, Charles Rosen ${ }^{2}$, James W. Simpkins ${ }^{3}$, Sanjay Bhatia ${ }^{2}$, and Dandan Sun ${ }^{4}$. Chapter three references material from the main body of work and was authored as follows: Gina Sizemore ${ }^{1}$, Sarah McLaughlin ${ }^{2}$, Mackenzie Newman ${ }^{3}$, Kathleen Brundage ${ }^{4}$, Amanda Ammer $^{2}$, Karen Martin $^{2}$, Elena Pugacheva ${ }^{5}$, James Coad ${ }^{6}$, Malcolm D. Mattes ${ }^{7}$, Han-Gang $\mathrm{Yu}^{3}$. Finally, chapter four supports the research with the transition to epidemiology and references material authored as follows: Gina Sizemore ${ }^{1}$, Toni Rudisill ${ }^{2}$.

In summary, this composition weaves a thread across the clinical translational science field intertwining chapter two (paper 1) a methods paper of ion channels and the electrophysiology tool, with chapter three (paper 2) the novel discovery of an ion channel target, and the epidemiologic opportunity for ion channel translation in chapter four (paper 3). 


\section{CHAPTER TWO}

\section{Temporal Lobe Epilepsy, Stroke, and Traumatic Brain Injury: Mechanisms of Hyperpolarized, Depolarized, and Flow-Through Ion Channels Utilized as Tri-Coordinate Biomarkers of Electrophysiologic Dysfunction}

The brain is an integrated network of multiple variables that when compromised create a diseased state. The neuropathology of temporal lobe epilepsy (TLE), stroke, and traumatic brain injury (TBI) demonstrate both similarity and complexity that reflects this integrated variability; TLE with its live human tissue resection provides opportunity for translational science to demonstrate scale equivalent experimentation between the macroscopic world of clinical disease and the microscopic world of basic science. The extended value of this research is that the neuroinflammatory abnormalities that occur throughout astrocytes with hippocampal sclerosis and damaged or even reversed signaling pathways (inhibition to excitation such as with gabaaminobutyric acid) are similar to those seen in post-stroke and TBI models. In evaluation of the epilepsy population this interconnectedness of pathology warrants further evaluation with collaborative efforts. This review summarizes patterns that could shift experimentation closer to the macro level of humanity, but still represent the micro world of genetics, epigenetics, and neuro-injury across etiologies of physiologic dysfunction such as TLE, stroke, and TBI with evaluation of cell function using electrophysiology. In conclusion we demonstrate the plausibility of electrophysiologic voltage and current measurement of brain tissue by patch clamp analysis to specify actual electrophysiologic function for comparison to electroencephalography in order to aid neurologic evaluation. Finally, we discuss the opportunity with multiscale modeling to display integration of the hyperpolarization cyclic-nucleotide gated channel, the depolarized calcium channels, and sodium potassium-chloride-one/potassiumchloride-two co-transporter channels as potential mechanisms utilized as tri-coordinate 
biomarkers with these three forms of neurologic disease at a molecular scale of electrophysiologic pathology.

Recent statistics indicate 50 million people suffer with epilepsy [1]. In adults with focal epilepsy, about $50 \%$ of them have had a previous brain injury [2]. Approximately $2.3 \%$ to $14 \%$ of stroke patients will develop seizures [3] and temporal lobe epilepsy (TLE) develops at rates inconsistent with heritability alone lending credence to underlying brain damage or otherwise dysfunction as a contributing feature [4]. A common feature in TLE, stroke, and traumatic brain injury (TBI) is the electrophysiologic dysfunction. This review will utilize the common thread of electrical biophysics to identify existing knowledge, expose the gaps, and project a plan for future research. Although the pathophysiology of epilepsy often begins with the discussion of genetic mutations, such as the SCN1A gene mutation with over 350 identified mutations [5], those mutations are not consistent across all epilepsy. For example, a specific mutation in the SCN1A gene, which affects an alpha1 subunit in the voltage-gated sodium channel, is not compromised in all epilepsies and not researched at all in diseases like stroke or TBI. Yet, SCN1A has demonstrated a phenotype associated with hemiplegic migraine [6], which is often a symptom in TBI. Newer research, at smaller scales, reveals mutations of a variety of genes that encode for signal transduction, synaptic transmission, neuronal metabolism and excitability, as well as brain development that play a role in disease pathology. In addition, variability in epigenetics distributes change that do not alter the original DNA, but create a disease state by alterations like methylation, and histone modification that can silence or overexpress proteins. Genes can be evaluated with specificity of their protein expression at the tissue injury region. These epigenetic contributors are demonstrated across a wide range of epilepsies and show correlation with encephalopathy seen across the neurophysiological and biophysical mechanisms of blood brain 
barrier compromise in stroke, ion channelopathy of TLE, and white matter apoptosis and necrosis of TBI. This review builds a plan to pull up from the microscopic and pull down from the macroscopic to define a place in the middle using a scale of disease evaluation that can be communicated between the discovery of basic science and the application of clinical medicine.

Despite this wealth of knowledge, greater than $30 \%$ of epilepsy patients continue to battle with a drug resistant seizure disorder while stroke and TBI patients bear the persistent symptoms related to the electrophysiologic dysfunction that ultimately reflects neurodegenerative change of varying degrees. Neuropathology of electrophysiologic dysfunction is complex and encompasses neuro-injury, epigenetics, and genetics. This complexity requires organization of scale to evaluate the interdependence and integration of these components. The excitability and inhibition balance required for homeostasis can be compromised by signaling cascades, genetics, or system failure across multiple neuronal circuits [7]. Therefore, selecting three diverse forms of electrophysiologic dysfunction: stroke, traumatic brain injury, and temporal lobe epilepsy - this review will discuss their commonality of electrophysiologic dysfunction, the limitations of the tools for evaluating electrophysiologic dysfunction, and the benefit of focused analysis with a hyperpolarized $(\mathrm{HCN})$ channel, a depolarized calcium $(\mathrm{CaV})$ channel and the sodium-potassiumchlorideone/potassium-chloride-two (NKCC1/KCC2) co-transporters. They can be correlated with the epigenetic expression, RNA and protein analysis to determine biomarkers for improved evaluation and targeted drug treatment of these diseases.

\section{Translational Research}

A challenge in translational research is application of precise, controlled basic science research to the multi-variable clinical reality. Therefore, evaluation across the three diseases of stroke, TLE, and TBI demonstrates how electrophysiology offers a broad level translational step 
between the complexity of the disease (clinical) and the specificity of individual dysfunctional components (basic science). This review utilizes location, morphology, and neuropathology to create a context for the discussion.

\section{Location}

Circulation to the brain arises from both the internal carotid arteries anteriorly and posteriorly through the vertebrobasilar artery system [8]. The hippocampus, the focal region of TLE, is fed through the posterior system with anterior contribution from the anterior choroidal artery. Although, not the only contributor to translational research shortcomings from mouse to clinic the vascular diversity requires consideration. The mouse does not evidence the anterior choroidal artery seen in humans, but with a smaller surface area reflects a more collateral system compared to the more end arterial system of humans. In Figure 1 you see the comparison of the mouse and human vascular system. The mouse brain $(1 \mathrm{~A})$ is created by a novel perfusion technique that allows resin casting of the mouse vasculature that maintains exact measurements of vascular diameter and length while eroding all other brain tissue to leave only a model of the vascular system, no brain tissue. In addition, the casting system maintains the network connections to demonstrate the collateral system of vasculature in the mouse brain. Everything in the picuture is vasculature. Compare that to the perfusion of the human brain (1B) in CT scan analysis. The end arterial system is a striking contrast to the more collateral system seen in the mouse vasculature. In (1C) the anterior choroidal artery is marked, as it is the only artery reaching to the hippocampus from the arterial system in humans, apart from the recurrent artery of heubner. Again, this is contrasted to the more collateral mouse vasculature to the hippocampus, which may afford it some neuroprotection during injury. It is important to continue animal model 
research. However, it is paramount that research of human tissue at this scale be developed to offset the limitations of animal models and build on their successes.

Major injury in stroke most frequently occurs from the anterior system, while TBI injury more frequently involves the posterior system through vertebrobasilar dissection. Therefore, TLE offers the opportunity of a control disease by virtue of consistent hippocampal location injury for evaluation with the variant anterior-posterior hippocampal injury from stroke and TBI. The anterior choroidal artery, a branch off the internal carotid, just distal to the posterior communicating artery is part of the anterior circulation, which runs deep to the hippocampal region. In addition, the recurrent artery of heubner originates from the anterior system. The variation between these two structures is consistent in that when one is dominate the other balances to a smaller development. The anterior choroidal represents part of the residual circulation from our evolutionary past before the posterior circulation developed with our higher cortical brain and therefore along with the heubner artery offers an opportunity to compare and contrast data with a mouse model in the evaluation of stroke, TBI, and TLE. Though absence of the anterior choroidal and heubner artery in mice is not the singular cause for the translational difficulty, research can demonstrate key functional variances between a collateral system (mouse) and an end artery system (human) in the circulation within a unique vascular region of brain.

Approximately two-thirds of ischemic stroke arises in the anterior circulation with $90 \%$ of those involving infarct of the MCA [9]. However, despite multiple animal models of MCA injury, we have failed to discover new drug therapy or targets that translate to successful clinical application. The anterior choroidal artery, which lies proximal to the MCA and the end artery structure of humans contrasted to the more collateral structure of mice may offer clues as science 
develops improved biotechnology to traverse these diverse pathways. In TBI, the most common vascular injury is subarachnoid haemorrhage [10] with secondary vascular issues from vasospasm or lumen narrowing of arteries from edema or increased intracranial pressure that further reduce brain perfusion [11]. Vertebrobasilar dissection is more common than anterior dissection in traumatic brain injury. The posterior vasculature diameter is narrow and causes difficulty, even under more stable situations such as bypass grafts where new techniques have been developed to overcome the disadvantage of these high-flow grafts due to the mismatch in vessel diameter between donor and recipient vessels in the posterior circulation [12]. With the hippocampal sensitivity to ischemia dissection in the posterior system leads to decreased perfusion by both blood loss and arterial narrowing due to acute spasm. The hippocampus is sensitive to ischemia and periods of excessive intracranial pressure and blood loss could demonstrate more significant injury in an end arterial human system compared to a more collateral system. Finally, TLE, which is noted for its hippocampal sclerosis, remains devoid of significant vascular evaluation despite our knowledge of glial cell function in maintenance of the blood brain barrier and its dysfunction in developing sclerosis. The brain vasculature represents the pathway in and out of the neurologic circuitry and is too broad to cover the entire network for all three diseases. Therefore, location will be restricted to the vascular distribution of the hippocampus which reaches deep into our evolutionary past, yet still offers parameters with defined limits for the discussion of stroke, TBI, and TLE within a framework that makes them consistent with one another by virtue of location. In addition, it addresses specific gaps in our knowledge of glial involved vascular injury in TLE, hippocampal role in cognitive and memory decline in TBI, and isolates a specific region for analysis of core versus penumbra injury in stroke. "The penumbra is characterized by the loss of action potential firing but maintenance of 
proper resting membrane potential" [13]. Resting membrane potential and action potential firing are key electrophysiologic targets translational research can utilize.

\section{Electrophysiology}

Neuroimaging is the gold standard evaluation in stroke and TBI and offers benefit in surgical planning with TLE. However, despite the benefits of these tools, they fail to establish data that connects structural abnormality and electrophysiologic dysfunction in the brain that translates to improved clinical outcomes [14]. EEG provides cortical activity markers in real-time [15] which augment the time lag in neuroimaging. However, few facilities have compatible tools to perform these tests synchronisly and have led to decreased use of electrophysiologic tools in preference to BOLD MRI, perfusion CT, and specialized manipulations of scans as accessories to primary MRI and CT for many neurologic diseases. Therefore, assessment of neuronal oscillations with electroencephalography and ion channel function is a novel way to assess the balance between excitatory and inhibitory cortical processes and provide biomarkers for future evaluation and treatment. The $\mathrm{HCN} 1$ channel, the $\mathrm{CaV}$ channel, and the $\mathrm{NKCC} 1 / \mathrm{KCC} 2$ cotransporter channels are representatives of hyperpolarization, depolarization, and flow through that can create a threeprong coordinate evaluation of current that can be used as a biomarker to aid evaluation and treatment being done with neuroimaging and electrophysiology tools such as EEG. In addition, it reflects the opportunity for bioengineering to develop alongside new electrophysiologic technologies such as microelectrode arrays, which improve spatial resolution over EEG. These data can be correlated with data from electrophysiology (See Figure 2). The glass pipette patching an astrocyte (2C) indicates the opportunity electrophysiology offers to bring the macroscopic world (2A) the glass pipette electrode and (2B) the microscopic world of an astrocyte together and produce (2D) research data in real time electric current evidence of cell 
function that communicates with a language that is the same in the macroscopic and microscopic world. Thus, creating the possibility to translate information between scales of the very small and precise (basic science discovery) and scales of the very large and complex (clinical application).

\section{Morphology}

Morphology evaluation is based on literature, which demonstrated large-scale involvement of astrocytes and pyramidal neurons in all three diseases. Astrocytes are an active and reactive component of the brain that influences gene expression, extracellular matrix components, and metabolism. When injured they display distinct features compared to healthy astroglia in their morphology, expansion, and signaling in ischemia [16] as well as TBI [17], and sclerosis in TLE [18]. Pyramidal neurons are the most common recipient within the environment of change commanded by astrocytes. Specific cell types vary by location which ultimately effects their function within those regions and the hippocampus remains a controversial region [19]. In addition, ion channel distribution on any given cell differs and contributes variation to the cell function. The pyramidal neurons of the CA1 region of the hippocampus are extremely sensitive to ischemia and ion channel research continues to evaluate this for mechanism though it is not yet well defined [20]. Unlike the L5 pyramidal neurons, which stretch through all five levels of the cortex, the CA1 pyramidal neuron structures remain confined to the CA1 hippocampal region and offer a specific region for discussion and experiment to isolate changes affected by stroke, TBI, and TLE. The diversity of cell structure contributes to the variety of function each cell offers to the brain (See Figure 3). It demonstrates the variance of a pyramidal neuron (3A); notice the CA1 and CA3 pyramidal neurons, of specific interest within the hippocampus, compared to the Layer V(LV) pyramidal neuron. In (3B) the LV pyramidal neuron with its streamline look reaches through all six layers of the neocortex, with little to no branching, while 
the more compact $\mathrm{CA} 1$ and $\mathrm{CA} 3$ of the hippocampus with their apical branching are designed for their local purpose. Research indicates a large percentage of $\mathrm{HCN}$ channels are expressed in the apical region of pyramidal neurons. The benefit of whole cell patch is it reads the resting membrane potential of the entire cell at the soma (3C). The alternate protocols available in whole cell patch, such as Ramp protocol (3D) and Pulse protocol (3E) allow evaluation of specific electrical changes in the entire cell. Therefore, the hippocampus, with the apical girth of CA1 and CA3 offers opportunity for significant evaluation with electrophysiology of these hyperpolarized channels in epilepsy and other neurologic disease.

Genetic and epigenetic modifications play a role in the channel construction, and expression of the channels discussed in this review. Within the spectrum of each disease the full context of those modifications is not yet understood. This review's purpose is to demonstrate the pathology based on the role of the channels within the cell for depolarization, hyperpolarization, and flow through or electroneutral current to open further discussion of experiment direction for disease specific research evaluating these channels as coupling or co-contributing channels in multiple disease pathology. $\mathrm{HCN}$ as a hyperpolarizing channel leads to hyperexcitability when it is mutated from its normal function, Ca leads to multiple variations of depolarization and intracellular activity when it mutates from its normal function, and the electroneutral cotransport channels already demonstrated as coupling with sodium and potassium alter their flow-through neutral status when they mutate from normal function. Approaching this from a predominantly electrophysiologic perspective puts focus on the function of the channels when they are mutated or have variant expression more than on the depth of discussion of each mutant variation. A mutation either causes the channel to work more effectively, less effectively, or not at all. Overexpression or underexpression similarly may impact function but, may also not affect cell 
function at all. This review aims to discuss the role of these channels in the broader terms of cell function in the context of disease rather than specific mutations for any gene family.

Figure 3D Demonstrates a Ramp protocol, which is the simplest screening tool to evaluate channels that open with depolarization. We have used this protocol in research to demonstrate how overexpression does not always tell you what the function will be in the cell. If there is overexpression of a specific channel on a scale of 1000 to 1 , yet only $5 \%$ of channels are open at the physiologic state then having overexpression of essentially 1000 doors does not matter. The function in the cell does not change if they are not open. Therefore, electrophysiology can give us functional data about what is going on in the cell without being distracted by genetic mutations that may pose no effect to the function or dysfunction of the cell in the disease. Yet equally, when there is abnormal function in the cell researchers can track that dysfunction back to its specific components of mutation and expression.

\section{Neuroinflammation}

The signaling pathways for neuroinflammatory response have been exposed as a contributor to pathology in neuro trauma, whether the acute injury of stroke, TBI, or the chronic sclerosis of TLE. Research linked NKCC1 signal increase to increased ischemic risk [22]. Inflammation of astrocytes, seen in temporal lobe epilepsy, occurs due to excess glutamate through the glutamine/glutamate/cycle (GGC) [18]. There is significant change demonstrated in the pathway from epileptogenesis through sclerosis in a hippocampal astrocyte cell. Comparable astrocyte damage occurs in post-stroke by compromise of the arteries where astrocytes assist in maintaining the blood-brain barrier and in TBI with similar compromise of the blood-brain barrier where in mouse models it is associated with memory changes [23]. There is glutamate uptake at the astrocyte where it is converted by glutamine synthetase to glutamine and released 
to the extracellular space. The neuron then uptakes the glutamine where it is degraded to glutamate by phosphate activated glutaminase (PAG) and released back to the extracellular space as glutamate to continue the cycle. There are multiple opportunities for the system to fail in this cycle from glutamine synthetase deficiency or glial glutamate (GLT-1) transporter dysfunction in the astrocyte to neuron dysfunction by excessive release due to (PAG) and the excitatory amino acid carrier (EAAC1); all of which contribute to extracellular/intracellular ion gradient shifts that disrupt homeostasis.

\section{Neurodegeneration}

Neurodegeneration is a natural path from the epigenetic contributions of neuroinflammation. The degeneration of neural tissue in neurologic disease is not singular in its origin and due to mixed contributions of neuroinflammation, gene expression, and ion dysregulation it is difficult to determine the cause and direction of pathology [24]. The epigenetic impact of the inflammatory process can create signal error or excess that leads to sclerosis and/or blood brain barrier breach [25]; just to name a few. New research indicates mitochondrial mechanism with miR-34 mediated regulation of the blood brain barrier and analysis with bioinformatics indicates several gene targets [26]. Research correlating upregulation and down regulation of co-transporter channels such as NKCC1 and KCC2 respectively following injury [27] support research of these channels in the pathology of stroke, TLE, and TBI where neuro inflammatory processes led to neurodegeneration. In addition, inflammation can produce neurodegeneration by abnormal gene expression, trigger regulating error, and channel surface expression variation with posttranslational modification. For example, the HCN1 channel has an exposed extracellular posttranslational modification where circulating inflammatory signals may contribute to dysfunction. Further review of a susceptible post-translational modification site exposed on the 
HCN1 channel can be found in Lee et al., 2017 [28]. These epigenetic influences of neuroinflammation can diverge from neurodegeneration and impact neurogenesis as well.

\section{Neurogenesis}

Increased ion channel density demonstrated significantly depolarized astrocyte cells in hippocampal foci damaged regions $(\sim 55 \mathrm{mV})$ compared with astrocytes in hippocampal neurotypical regions $(\sim 75 \mathrm{mV})$ since early channel research [29]. This channel density neurogenesis continues in recent research, most notably in new research regarding $\mathrm{NKCC} 1$ and KCC2 co-transporters where ratio shifts to embryologic conditions indicate again the role of channel density in the probability for dysfunction [30]. In addition, the electrophysiologic effect of channel density on surface expression, post-translational modification, and signaling demonstrate the interconnectedness and circular difficulty of the pathology. The anticipated voltages can be calculated by Nernst equation demonstrating the polarity shift that the ratio inversion of NKCC1 to KCC2 could create in the membrane potential (see Figure 4). The Nernst equation in figure four demonstrates the role that concentration of a single ion can play in altering the membrane potential of a cell. With so many basic science variables from gene expression to neuroinflammation, neurodegeneration, and neurogenesis it is necessary to find a system that reflects the function of them all. Electrophysiology can extract the functional data that in turn can be correlated with microscopic science and macroscopic disease.

\section{Ion Channel Milieu}

Regulation of ion homeostasis is critical to the function of cells. Ion transporters establish that by cell volume, voltage influence of membrane potential, and signal transduction [31]. Shifts in cellular volume and gradient pressures trigger homeostatic responses coordinated by ion 
channels to mediate and avoid excessive cellular edema or dehydration with regulatory volume adjustments [32]. Membrane potential is primary in the discussion of cell survival and function. Therefore, membrane potential is the necessary starting point. The HCN1 channel with its Ih funny current displays a reversed polarity in its biophysical nature with a reversal potential $\sim-20$ $\mathrm{mV}$ carrying a cation current of both $\mathrm{Na}+$ and $\mathrm{K}+$ that is inward moving at rest [33]. This combination along with additional features to be discussed creates an intersection of opportunity for interaction with sodium-potassium-chloride cotransporters NKCC1/KCC2. Signal transduction pathology disrupts cell homeostasis. It is here where ion channels can create significant influence. GABA is one of the most researched contributors to signaling pathways and demonstrates the importance of ion channel interaction in the discussion of negatively charged ion concentrations, such as $\mathrm{Cl}^{-}$within the cell membrane [34].

\section{Hyperpolarized Cyclic-Nucleotide Ion Channels}

Sequencing, Construction, and Function

$\mathrm{HCN}$ role in epilepsy is a function of its isoform's homomeric or heteromeric channel construction, expression, and interaction. There are four HCN isoforms: HCN1, HCN2, HCN3, HCN4. The expression of HCN1 is more prevalent in the hippocampus and cortex compared with the $\mathrm{HCN} 2$, and $\mathrm{HCN} 4$ isoforms, which are more prominent in the thalamus and sinoatrial node [35]. In discussing the role of $\mathrm{HCN}$ channels in epilepsy it is not a singular mutation of the channel but rather a combination of channel construction variance as well as altered expression which demonstrated epileptic disease in mouse models [35]. In addition, deletion of HCN1 and HCN2 in knock-out mice created seizure prone mice [36]. 
However, the structure of the HCN1 channel is showing significant promise for integrated roles with its reversed polarity [37]. The hyperpolarization as opposed to depolarization gating make the HCN1 channel an excellent candidate for integrated research with NKCC1 and KCC2 cotransporter channels along with the depolarization gating of the high voltagegated $\mathrm{CaV}$ channels. The construction of the HCN1 channel pore has great conservation with that of the K+ channels; both contain the glycine-tyrosine-glycine (GYG) as a selectivity filter [38]. The function of the $\mathrm{HCN} 1$ channel is to operate with reverse polarity and it does this by structural design that allows for conformational changes of the pore based on the trigger mechanism. Using cryo-electron microscopy (Cryo-EM), researchers demonstrated the alternated gating between hyperpolarization and cAMP modulation [37]. We hypothesize that hyperpolarization channels like $\mathrm{HCN} 1$ in conjunction with inappropriate $\mathrm{NKCC} 1 / \mathrm{KCC} 2$ co-transport channel ratios allows function gain or inhibition at a more negative membrane potential leading to hyperexcitation, epileptogenesis, neuroinflammation and therefore has a high potential link to hippocampal astrocytic and pyramidal neuron injury with evidence of electrophysiologic dysfunction either as neurodegeneration, hyperexcitation or both in stroke, TBI, and TLE. HCN produces a current called funny current, If , in the heart and is considered the pacemaker current. It is often written as Ih when discussing its current in the brain. The Ih current is an inward current [39]. Its complexity of construction from homomeric to heteromeric, as well as expression of the channel, impacts how closely to resting cell membrane potential the channel can be triggered. Additionally, reduction in expression as well as complete deletion such as is done in knockout mice consistently indicates increased seizure risks and activity in other neurologic disease such as stroke and TBI. 
Channel electrophysiology connects with epilepsy, stroke, and traumatic brain injury by offering a context of functional evaluation to these diseases. Each disease has pathology rooted in failing cellular processes of which ion channels are a key component. When using an evaluation of scale as macroscopic as a complete human disease it is difficult to correlate it with equal scale at the basic science level (i.e. discuss the function of a cell, as a whole, rather than a single variable of change such as a histone modification that changes the expression of a gene). Each of these individual discoveries is important. However, electrophysiology of cell function creates a closer equality of scale for translational science because the microscopic data is measured, discussed, and analyzed with the same tools as the macroscopic data. Resting membrane potential, depolarization, and hyperpolarization states are mandatory tools for a cell's homeostatic balance. In this review we press for the whole homeostatic electrical balance system of the cell to be used for representation of the larger scale human disease. It is not equivalent, but it is a starting point to begin the translational discourse between clinical and basic science. Ion channels play a role in the diverse signaling throughout stroke injury at glial cells, neurons, and the blood brain barrier [40]. Traumatic brain injury leads to variant excitotoxicity, which could be evaluated, with electrophysiology to better understand the functional changes at the cellular level [41]. Epilepsy results in hyperexcitability recorded by electroencephalography and confirmed in electrophysiology recording baths at the cellular level by evaluation of ion channel function [42]. Experimentation to evaluate these diseases for similarities with channels specific for these electrical potentials can establish a baseline for deeper discovery of the disease process. Electrophysiology is the translational lane of research that is yet to be completely developed and explored; a lane that runs like a blood brain barrier between the worlds of clinical research and 
basic science (See Table 1). This electrophysiologic diversity can be evaluated further with future research since evidence for all four $\mathrm{HCN}$ isoforms in the brain have been described [43].

\section{NKCC1 and KCC2 Co-Transporter Ion Channels}

Sequence, Construction, and Function

Research on variants have associated R952H and R1049C of the KCC2 co-transporter channel with idiopathic generalized epilepsy (IGE) [45] a more generalized epilepsy. KCC2 plays a dominant role in normal development, influencing factors such as the excitatoryinhibitory signal read of GABA [46]. When $\mathrm{KCC} 2$ is deficient, especially in relevance to $\mathrm{NKCC} 1$, brain development is compromised. Intracellular chloride currents show biophysical accumulation when the ratio construction of NKCC1 expression is higher compared to KCC2 expression [47]. This leads to severe neurologic dysfunction and delay noted due to decreased function of the $\mathrm{NKCC} 1 / \mathrm{KCC} 2$ co-transporter's ability to maintain low intracellular Cl- because of mutations at SLC12A5 [48]. The fundamental role of $\mathrm{Na}-\mathrm{KCl}$ cotransporter 1 (NKCC1) and $\mathrm{K}-\mathrm{Cl}$ cotransporter 2 (KCC2) is lost when the expression of each is not regulated properly. In addition, the integrated signaling system as it relates to GABA is disrupted [49]. The NKCC1 and KCC2 provide the $\mathrm{Cl}$ - homeostasis voltage within the cell [50], which is why they are pertinent to evaluation of hyperpolarization-gated channels like HCN1.

The deregulation of NKCC1 in epilepsy is demonstrated by the increased association with epileptogenesis due to its role in managing the concentration of intracellular chloride [51]. The slc12 gene family encodes both $\mathrm{NKCC} 1$ and $\mathrm{KCC} 2$ and mutations in this gene have been associated with epilepsy. Many of these electroneutral chloride cotransporters functionally 
couple movement of chloride with Sodium and potassium and have demonstrated pathological hyperexcitability in mice with genetic modifications of this gene [52].

In models of evaluation these chloride channels demonstrate the detrimental effect of neuroinjury on their ratios to one another. Multiple research platforms including ischemic stroke, TLE, and subarachnoid hemorrhage (the most common brain injury in TBI) reveal the correlated role between disease and damage in these channels. Western blot analysis by Yong Tien, et al (2015) revealed multiple neuroinflammatory components following subarachnoid hemorrhage. The $\mathrm{NKCC} 1$ and $\mathrm{KCC} 2$ ratio shifts were significant, demonstrating changes of their protein expression [53]. Western blot analysis characterized a decline in KCC2 expression and increase expression in $\mathrm{NKCC} 1$ following induced subarachnoid hemorrhage in their rat model. Topiramate, a frequently used anticonvulsant, effectively reversed those KCC2/NKCC1 expressions in a dose-dependent fashion. This response is a positive indicator of pharmacologic opportunity as we learn more about the integration of these channel functions.

\section{High Voltage-Gated Calcium Ion Channels}

High voltage-gated calcium ion channels are discussed briefly below due to their connections to epilepsy and the role they could play in the development of an integrated computational model of ion function for future research.

HVA calcium channels have five known types, all derived with an original subunit: CaV1 the Ltype, $\mathrm{CaV} 2$ including the P/Q-type [CaV2.1] the R-type [CaV2.2] and N-type [CaV2.3], and the CaV3 the T-type. These types have been studied with regards to genome structure, mutation, pathology, and pharmacology. The results thus far are promising. The P/Q type is the CaV2.1 type of the five types and has the most connection with epilepsy along with the CaV3.2 T-type. 
They both are linked to IGE. The HVA channel requires higher depolarization, compared to LVA channels. Yet, both are linked to IGE, both are pharmacologically responsive, and have improved epilepsy outcomes with inhibition in patients [54]. Low Voltage Gated Channels like the T-type channels offer the first glimpse of opportunity for hyperpolarized channels and negative charge transporters to be linked to IGE. The structural changes and pathology of these channels is tied to congenital mutations associated with IGE which raises the question for susceptibility to more epigenetic, neuroinflammatory changes linked with more focal epilepsy like TLE, post-stroke epilepsy (PSE), and post-traumatic epilepsy (PTE). The voltage-gated channels have many polymorphisms that contribute to the mutations leading to structural and functional change [55], but alone they do not answer all the questions regarding electrophysiologic dysfunction.

The CaV families, which are the L-type channels [CaV1], P/Q-type [CaV2.1] (R-type and Ntype of $\mathrm{CaV} 2$ family not reviewed here), and T-type [CaV3] channels, have defined structure. The $\mathrm{CaV} 1$ and $\mathrm{CaV} 2$ types have an alpha subunit that forms the pore of the channel and a gamma, beta, and alpha2delta unit forming the tertiary structure. These channels are closely related to the $\mathrm{CaV} 3$ channel, which also has an alpha unit pore [56]. Splicing variants of each subunit contribute to altered phenotypes associated with epilepsy [57]. These splice variants determine biophysical properties and tissue location of channels [58].

Current data from both animal models and clinical trials point to CaV2 the P/Q type HVA channel, and the CaV3 T-type LVA channel as important, but not alone in their epileptogenesis role. These channels are well studied and presented briefly here due to their strong link to IGE that could shed light on the genetic contributions to the electropyysiologic dysfunction of TLE, stroke, and TBI. In addition, the role of calcium in contributing to glutamate excitotoxicity is 
relevant [38]. Whether from ischemia, TBI, or epilepsy the rich research database on HVA calcium channels can help form a background for baseline computational modeling regarding ion gradients.

\section{Clinical Relevance and Conclusion}

The animal models for stroke, TBI, and TLE are beneficial to science and humanity in our growth of knowledge, yet they often fail to translate to improved outcomes in human clinical applications. Animal models have demonstrated multiple failures in producing drugs with safety and efficacy in humans despite success in preclinical experiments [59]. What are the limitations that contribute to this discrepancy in translation from bench science to clinic?

\section{Limitations of Electrophysiologic Tools}

Unlike Electrocardiograms (ECG/EKG), which are standard of care in heart attack evaluations; stroke, with its similarities of ischemia, rarely receives evaluation by an electroencephalogram (EEG) until the following day, if at all. EEG gives an electrical reflection of the brain's activity. In focal epilepsy, such as TLE, that can be focal waves or spikes [60]. In addition, electrical abnormalities consistent with encephalopathy are often seen in stroke patients when EEG is performed. Yet, many rural hospitals do not utilize EEG to monitor patients with these neurologic conditions because of limitations in skilled personnel and of the equipment itself. Whether evaluation is in a mouse model or a hospital, the spatial discernment sacrificed by electroencephalography due to the bony scalp and other confounding obstacles between the electrodes and the signal cannot always be overcome by the timing integrity of the machine. Event related potentials, frequency spectrum analysis, and other mechanisms of analysis assist in 
overcoming these limitations [15]. However, training and validation of the researcher's technique can consume large amounts of research time.

\section{Overcoming EEG Limitations}

Microelectrode arrays and local field potentials show promise in the prediction and localization of preictal activity prior to seizures in TLE [61]. As these tools develop new limitations emerge. For example, a micogrid covers a large surface area in a mouse, but in a human in is a very small area for analysis. This is where the growth of molecular biology and electropyhsiology targets the analysis of these small areas with the precision of basic science. With these developing technologies, the opportunity for molecular biology to create biomarkers of electrophysiologic dysfunction in diseases such as TLE, stroke, and TBI is present and could prove to be the translation required to bring bench science more effectively to the clinic.

\section{Pharmaceutical Limitations}

Anticonvulsant pharmaceutical products create the first line of defense for clinicians treating patients with epilepsy. However, many of the medications are non-linear in their kinetics which means their half-life and clearance are dependent upon plasma concentrations which can fluctuate, reducing the ability to maintain steady state of the drug. In addition, anticonvulsants such as Valproic acid, and Clonazepam are greater than $88 \%$ protein bound so they do not cross the blood brain barrier well and therefore provide little if any medication to the targeted areas. In addition, Valproic acid and similar drugs demonstrate serum protein saturable binding which can impact other medications when given simultaneously [62]. Newer anticonvulsants such as ethosuximide and topirimate are more promising, yet the disease is far from controlled. Stroke pharmaceutical options are more limited in that after decades of research we have essentially one 
medication; tissue plasminogen activator (tPA) that can be used in stroke and that is limited to specific time frames, and patient types. Finally, in TBI there are multiple complicating factors with medication management due to the complexity of symptoms from post-traumatic stress disorder to epilepsy.

Therefore, improved target evaluation and treatment based on a demonstrated abnormality of electrophysiologic dysfunction offers a significant opportunity to identify biomarkers for bioengineering and innovative technologies for multiple neurologic diseases.

\section{Ion Channel Options}

Ion channel targeting offers unchartered opportunity for further evaluation and treatment for TLE, stroke and TBI. Research demonstrates the adaptation of neurons across cellular types[63]. The integration of channel structure and function with cellular stability is described and accepted across research fields, though all the intricacies of how that works are not yet understood.

Continuing the process of collaborative research evaluating the interactions and correlations of multiple variables from genetics to inflammatory response by precise research of atomic chemistry at the ion channel level can expose those unknowns at the molecular level. In addition, the collaboration of scientists and technology can compile research data that can be integrated at unprecedented speeds and with increased safety. The Department of Defense, National Institutes of Health (NIH), and other large organizations recognize the increasing need for better outcomes for patients suffering with neurologic diseases like TBI, stroke, and TLE. NIH published a notice in the NIH guide in 2011 encouraging the scientific community to address the issues of predictive failure in preclinical research [64]. The focus to bring basic science to the bedside more efficiently, safely, and effectively creates an opportunity for research that can begin at this primary level and build forward. Evaluating the process of interaction between hyperpolarized 
HCN1 channels with its unique polarity, depolarized calcium channels, and NKCC1/ KCC2 ratio shifts in neuropathology creates a single variable of interaction that can be modeled in a multiscale modeling system with a designed control of homeostasis juxtaposed to the actual cellular expression identified by genetic analysis of diseased human tissue. The single constant of a homeostatic model that is computerized and easily modified as new data arrives lends itself not only to comparison against disease tissue, but also better understanding of homeostasis as we start to define the variation within normal. It is against this contrast that we will bridge the gap from bench to bedside and create - in real time - questions that can be answered safely and with better precision before heading to animal and clinical labs for experimentation. This can reduce the time to successful treatment in clinical application and increase efficacy and safety as we identify potential mechanisms that lead to neurodegenerative changes. TLE hippocampal sclerosis, ischemia in stroke, and blood brain barrier compromise in TBI can establish a baseline evaluation of electrophysiologic dysfunction. Then interaction and interdependence of the HCN1 channel, $\mathrm{CaV}$ channel and the $\mathrm{NKCC} 1 / \mathrm{KCC} 2$ co-transporter channel can correlate dysfunction with structure.

\section{Building A Mouse Model}

Location/Vascular - A global stroke model in mice creates hippocampal ischemia by way of the posterior circulation consistent with cardiac arrest as well as stroke. Mice have more collateral vascular systems unlike the end artery system in humans and the divergence of humans from that evolutionary past may hold one of the keys to improved biomarker targeting for humans. Therefore, comparison of the mouse model hippocampal data to human supplemental data can expose mechanisms for translational failures of mouse stroke models to clinical 
application and open doors for other neurologic disease discussion such as epilepsy and TBI as discussed below.

Morphology - Pyramidal neurons and astrocyte immunohistochemistry evaluation within the hippocampus can be used to demonstrate where - on the cellular structures - inclusion of HCN1 channels in control, ischemic injury mice, and hemorrhagic injury mice occurs. Further immunohistochemistry evaluation of mice from control, ischemia, and hemorrhagic stroke groups, triggered to seizure with kainic acid, can be analyzed for HCN1 channel expression prior to and after seizure. This further characterizes location and identifies spatial relationships for comparison to later human research. HCN1 channels with their extracellular exposed posttranslational modification tail may demonstrate significant changes and prove a key biomarker to stabilize the first coordinate of a tricoordinate system.

Structure - mRNA sequencing and Western blot for protein expression of HCN1 will reveal increased or decreased expression changes in hippocampal regions exposed to specific injury.

Function - Acute fresh tissue for electrophysiologic evaluation with specific voltage data by patch clamp on dissected hippocampal tissue can then deliver functional data to correlate with specific structure, morphologic, and anatomical data. This precision analysis creates the foundation for a model of translational human tissue analysis.

\section{Supplemental Future Research}

\section{Human Research}

Electrophysiology State of the Art Technique - IRB approved human research that can safely evaluate human tissue electrophysiologicaly from initial injury (EEG), through craniotomy and intraoperative electrophysiologic monitoring (intraoperative EEG) during tissue resection. 
Discarded tissue can then be evaluated for its voltage and current by patch clamp analysis to specify actual electrophysiologic function for comparison to macro tool evaluation. That electrophysiologic data can then be connected with the structural expression of biomarker channels demonstrated in spatial localization by immunohistochemistry and total expression of mRNA and protein by RT-PCR and western blot.

\section{Building the Future}

Multi-scale Modeling - Data programmed from the evaluation of the HCN1 hyperpolarizing channel, and additional channels such as the depolarizing calcium channels, and the flow through NKCC1/KCC2 co-transporter channels to demonstrate what is seen experimentally. This programmable data can then be used in simulations of tri-coordinate biomarkers for targeted cell therapy as a translational tool for future experiments prior to clinical application trials.

\section{Summary}

In summary, this reflects the limitations and difficulties of translation between the microscopic bench science and the macroscopic world of clinical disease. The need for clinical translational science evidenced by the 17 -year delay between bench and bedside ${ }^{1}$ is more evident than ever. Electrophysiology offers that translation because it communicates across measures of scale. Depolarization in the cell's resting membrane potential is represented the same way as depolarization on an EEG or EKG. It is this connection of depolarization that will be discussed in the next chapter both at the microscopic level of basic science and at the macroscopic level of disease. In chapter three you will see electrophysiology bridge the translational gap when a microscopic ion channel proves to be an electrophysiologic target for treatment of a deadly macroscopic clinical disease called triple negative breast cancer. 


\section{CHAPTER THREE}

\section{Opening Large-Conductance Potassium Channels Selectively Induced Cell Death of Triple- Negative Breast Cancer}

Background: Unlike other breast cancer subtypes that may be treated with a variety of hormonal or targeted therapies, there is a need to identify new, effective targets for triple-negative breast cancer (TNBC). It has recently been recognized that membrane potential is depolarized in breast cancer cells. The primary objective of the study is to explore whether hyperpolarization induced by opening potassium channels may provide a new strategy for treatment of TNBC.

Methods: Breast cancer datasets in cBioPortal for cancer genomics was used to search for ion channel gene expression. Immunoblots and immunohistochemistry were used for protein expression in culture cells and in the patient tissues. Electrophysiological patch clamp techniques were used to study properties of BK channels in culture cells. Flow cytometry and fluorescence microscope were used for cell viability and cell cycle studies. Ultrasound imaging was used to study xenograft in female NSG mice.

Results: In large datasets of breast cancer patients, we identified a gene, KCNMA1 (encoding for a voltage- and calcium-dependent large-conductance potassium channel, called BK channel), overexpressed in triple-negative breast cancer patients. Although overexpressed, 99\% of channels are closed in TNBC cells. Opening BK channels hyperpolarized membrane potential, which induced cell cycle arrest in G2 phase and apoptosis via caspase-3 activation. In a TNBC cell induced xenograft model, treatment with a BK channel opener significantly slowed tumor growth without cardiac toxicity. 
Conclusions: Our results support the idea that hyperpolarization induced by opening BK channel in TNBC cells can become a new strategy for development of a targeted therapy in TNBC.

\section{Background}

The main molecular subtypes of breast cancer are termed Luminal A (ER+/HER2-), Luminal B (ER+/HER2+/-, higher histological grade, more aggressive than Luminal A), HER2enriched (ER-/HER2+), and triple-negative (ER-/PR-/HER2-) [1]. Recent gene expression studies further identified five "transcriptional subtypes" of breast cancer: basal-like, HER2-enriched, luminal A, luminal B, and normal-like (now thought not to originate from breast cancer) [2]. Up to $70 \%$ of triple-negative breast cancer (TNBC) have the basal-like gene expression signatures, however, a large number of basal-like tumors express ER, PR or HER2 [3]. Further studies on molecular signatures, genetics, and genomics have led to the identification of four TNBC subtypes (basal-like 1, basal-like 2, mesenchymal, and luminal androgen receptor) [4, 5]. These studies have revealed the complexity of breast tumors and generated many new hypotheses for potential therapeutic targets for treatment of TNBC.

TNBC is one of the subtypes of breast cancer with an earlier onset, more aggressive metastasis, and lacks the therapies available to ER+, PR+, and HER2+ breast cancers [3]. Fiveyear breast cancer survival is significantly reduced with diagnosis of TNBC [6], due largely to relatively ineffective therapeutic options [7]. There is therefore a need to identify new, effective targets for TNBC.

All cells maintain a polarized membrane potential (Em), more negative inside than outside the cell membrane. Em is essential to the development of action potentials in excitable cells such as neurons and cardiac myocytes. However, accumulating evidence has also demonstrated 
variability of Em in non-excitable epithelial cells and cancer cells as well [8]. Alterations in Em (depolarization - i.e. Em becoming more positive, hyperpolarization - Em becoming more negative) are now recognized to play a crucial role in controlling the cell cycles $[9,10]$.

Using a traditional microelectrode technique, Em was reported to be $-13 \mathrm{mV}$ in breast cancer biopsy specimens from nine women with infiltrating ductal carcinoma, independent of ER or PR presence [11]. For comparison, normal human breast epithelial cell Em is near -60mV [11]. Thus, Em is depolarized in breast cancer compared to normal breast cells. Using whole-cell patch clamp, we found more positive Em in TNBC MDA-MB-231 cells $(-39.5 \mathrm{mV})$ than in normal breast cells $(-66.9 \mathrm{mV})[12]$

KCNMA1 gene encodes the pore-forming alpha subunit of a voltage- and calcium-gated large-conductance potassium channel, called BK (also known as Slo1, Maxi-K, or KCa1.1) channel $[13,14]$. Previous studies have suggested a contradictory role of KCNMA1 in breast cancer proliferation, invasion, and metastasis. Blockade of BK channels can slow proliferation and invasion of breast cancer cells $[15,16]$. In contrast, studies with anti-tumor compounds revealed anti-tumor action as an important result with activation of BK channels in metastatic breast cancer cells [17].

BK channels have to open (activate) to exert their function - hyperpolarizing Em by loss of intracellular potassium ions. Therefore, high expression of KCNMA1 does not necessarily guarantee high activity because closed channels do not have activity and cannot hyperpolarize the cell. Similarly, low expression of KCNMA1 expression levels can have a significant impact if all channels are open. Therefore, the strategy to inhibit or activate BK channels can only be decided after we determine if the channels are open or closed at the Em of TNBC cells. 
In this work, we present evidence for overexpression of KCNMA1 in TNBC from a large dataset. Second, we verify a significant increase in protein expression of BK channels in TNBC cell lines and primary TNBC tissues. Third, we provide an answer to an intriguing question regarding how breast cancer cells remain depolarized while overexpressing a hyperpolarizing ion channel, which should make cell membrane potential more negative. Fourth, we demonstrated that opening BK channels hyperpolarizes Em and induces apoptotic death of TNBC cells via apoptosis. Fifth, we show that BK channel openers can slow tumor growth in an MDA-MB-231 xenograft model in female NSG mice to validate the main in vitro finding. Finally, we show that this new approach of using BK channel openers for selective induction of death in TNBC does not impact healthy breast tissue and cardiac function.

\section{Methods}

KCNMA1 gene expression in The Cancer Genomic Atlas (TCGA) database

KCNMA1 gene expression patterns in primary breast cancer database were analyzed from The Cancer Genome Atlas (TCGA) [18] via cBioPortal (http://cbioportal.org ) [19]. Gene expression levels from RNA-sequencing data was illustrated in $\log 2$-fold of fragments per kilobase of transcript per million (FPKM) [20].

\section{Cell culture and plasmid transfection}

Human breast adenocarcinoma cells (ER+ MCF7, triple-negative MDA-MB-231/Luc and SUM159) were grown in Dulbecco's modified Eagle's medium (DMEM, Invitrogen), whereas triple-negative HCC1143 cells were grown in RPMI (Invitrogen), supplemented with 10\% fetal bovine serum, 1X Pen/Strep (Gbico 15140). Normal human mammary epithelial cells (MCF10A) were grown in mammary epithelial cell basal medium (MEC), supplemented with MEC growth 
kit (ATCC). Rat h9c2 cardiac myocytes were purchased from ATCC (CRL-1446), cultured in DMEM.

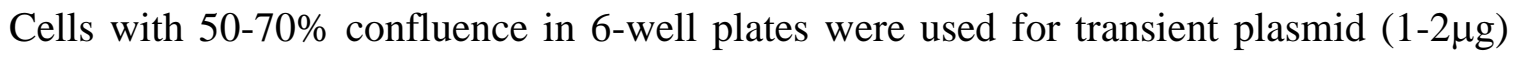
transfection using Lipofectamine3000 transfection reagent (Invitrogen). Human wild type (hSlo1 WT) and A313D mutant (hSlo1-A313D) channel cDNA in pcDNA3 mammalian expression vector were co-transfected with GFP for verification of expression.

Patch clamp studies in MDA-MB-231

Details in whole-cell or perforated (or permeabilized) patch clamp studies in isolated cells have been previously reported $[21,22]$. Briefly, the cells grown on coverslips were placed in a lucite bath with the temperature maintained at $35^{\circ} \mathrm{C}-37^{\circ} \mathrm{C}$. Em and voltage-gated potassium currents were recorded using the whole cell patch clamp technique with an Axopatch-700B amplifier. Em was measured with DMEM or Tyrode solution and pipette solution. DMEM contained physiological ion concentration (in $\mathrm{mM}): 150 \mathrm{Na}^{+}, 5 \mathrm{~K}^{+}, 2.0 \mathrm{Ca}^{2+}(\mathrm{pH}=7.4)$. Tyrode solution contains (mM): $\mathrm{NaCl} 140, \mathrm{KCl} 5.4, \mathrm{CaCl}_{2}$ 1.8, $\mathrm{MgCl}_{2}$ 1, Glucose 5.5, Hepes 5, pH 7.4 adjusted by $\mathrm{NaOH}$. The pipette solution contained (in $\mathrm{mM}$ ): $85 \mathrm{KCl}, 40 \mathrm{~K}$-Aspartate, $0.1 \mathrm{CaCl}, 10$ HEPES, pH was adjusted to 7.2 by $\mathrm{KOH}$. The pipettes had a resistance of $2-5 \mathrm{M} \Omega$ when filled with pipette solution. For perforated patch, amphotericin - B was added to the pipette solution to a final concentration of $240 \mu \mathrm{g} / \mathrm{ml}$. The whole-cell/perforated patch clamp data were acquired by CLAMPEX and analyzed by CLAMPFIT (pClamp 9, Axon/Molecular Device).

\section{Live cell imaging}

Live cell imaging experiments were performed using a Zeiss Axio Observer A1 inverted microscope with fluorescence. Images were acquired and analyzed using AxioVision (version 4.6). 
We used ethidium homodimer-1 (EthD-1, Invitrogen, $0.2-0.5 \mu l$ of $2 \mathrm{mM}$ stock to $1 \mathrm{ml}$ culture of cells in 6-well plates) to label dead cells. Cell count was performed using ImageJ (NIH).

\section{Western blotting}

Cells were harvested using Radioimmunoprecipitation Assay (RIPA) buffer with 1\% protease inhibitor cocktail (Sigma). We then sonicated lysates on ice and centrifuged at 12,000xg for 10 minutes at $4^{\circ} \mathrm{C}$. Tumor tissue was homogenized in RIPA buffer with $1 \%$ protease inhibitor cocktail (Sigma), then centrifuged at $12,000 x g$ for 10 minutes at $4^{\circ} \mathrm{C}$. Supernatant was isolated from debris pellet.

Protein concentration was measured using Bicinchoninic acid assay (BCA) (Thermo Fisher). Once protein concentrations were normalized across samples, they were then heated for 12 minutes at $90^{\circ} \mathrm{C}$. Samples were loaded into NuPage $4-12 \%$ bis-tris gels (Invitrogen) with MOPS running buffer at 70 volts for 100 minutes, then transferred to $0.2 \mu \mathrm{m}$ pore PVDF membrane (Thermo Scientific) at 30 volts for one hour in cold room. Next, blots were blocked in Licor blocking buffer for one hour, and incubated for 12 hours at 4 degrees with primary antibody for either anti-KCNMA1 for epitope 199-213 (Alomone cat\# APC-151), anti-caspase-3 (Cell Signaling Technology), or anti-Slo1 for c-terminus segment 9-10 (Millipore) at 1:500 dilution. The membrane was then washed 3 times for 15 minutes with tris-buffered saline containing $0.1 \%$ tween-20 (TBS-T). A secondary antibody, IR $800 \mathrm{CW}$ from Licor (1:20,000 dilution) was incubated with the membrane at room temperature for one hour. After three 10-minute washes with TBS-T, blots were imaged using a Licor Odyssey CLx and image studio software. If residual background signal was observed, additional washes of 5 to 10 minutes with TBS-T were 
completed and the membrane was re-imaged. Beta-actin primary antibody (Proteintech) and IRDye 680RD secondary antibody (Licor) were used as a loading control.

\section{Immunohistochemistry of patient breast samples}

Experiments involving patient breast samples were approved by West Virginia University Institutional Review Board. Formalin-fixed paraffin-embedded (FFPE) breast tumor tissue from patients was processed according to vendor's manual instruction (Biocare) and following a verified protocol in the Pathology Laboratory of Translational Medicine at WVU. Briefly, $3 \mu \mathrm{m}$ sections were deparaffinized on slides, quenched with hydrogen peroxide, and incubated in BK channel antibody (Sigma-Aldrich, HPA054648) Sigma at $4^{\circ} \mathrm{C}$ for 4 minutes. Horseradish peroxidasecontaining secondary antibody (UMap anti-RB, Roche, Diagnostic, Cupertino, CA) was then added for 8 minutes and developed using Biocare DAB (brown color). Hematoxylin was used as a counterstain (blue color).

Automated formalin-fixed, paraffin embedded immunohistochemical staining, to evaluate tumor antigen expression profiles, is available via the Ventana DISCOVERY automated IHC Platform. Breast tumor IHC slides stained with BK channel antibody were examined under an Olympus VS-120 slide scanner with a 10X Plan S Apo/0.40 NA objective, equipped with a color camera (Pike 505C VS50). The images were analyzed using OlyVIA (Olympus) and ImageJ (NIH). Percentage of area staining was used to quantify the protein expression levels in IHC slides. Breast tumor induction in NOD scid gamma (NSG) mice

Female NSG-immunodeficient mice of 4-6 weeks old were purchased from the Jackson Laboratory. Experimental procedures and housing of the animals were approved by the Institutional Animal Care and Use Committee. Animal were housed in a fully state-of-the-art facility that includes large specific pathogen free rooms, husbandry conditions (breeding program, 
light/dark cycle, temperature control, quality water, clean cages access to food and water), and welfare-related policies related to tumor studies (e.g., tumor burden policy).

Following power analysis, a total of 16 female mice was used, 8 for treatment, 8 for controls. For mammary pad injections, pathogen-free luciferase-expressing human breast adenocarcinoma cells (MDA-MB-231/Luc, 1-2x10 cells/animal) were injected into the fourth inguinal mammary gland of 6- to 8-week-old mice. Primary tumors had formed typically two weeks following cell injection. Tumor size was monitor by imaging twice a week. After experiment, mice were euthanized by isoflurane overdose (5\% to effect or an overdose $100 \mathrm{mg} / \mathrm{kg}$ of sodium pentobarbital), a procedure approved by our IACUC.

Ultrasound imaging of xenograft tumor in NSG mice

Details for ultrasound imaging of xenograft tumor in NSG mice have been previously reported [21]. Briefly, animals were anesthetized by exposure to 1-3\% isoflurane during imaging. Imaging was performed weekly over the course of each experiment, typically for 4-6 weeks. Tumor volume was imaged by ultrasound imaging (USI) with Vevo2100 Micro-Ultrasound System. A 40 or $50 \mathrm{mHz}$ transducer was used, depending on the tumor volume. A 3-dimensional (3D) image was acquired with scanning distance of $0.071 \mathrm{~mm}$ between images. Vevo software then integrated the images into a reconstructed 3D tumor from which the tumor volume was obtained.

BK channel openers

BMS-191011 (Tocris) and NS11021 (Tocris) were prepared in 10mM DMSO stock. The working concentrations of $10-50 \mu \mathrm{M}$ contained $1-5 \mu \mathrm{l}$ of DMSO in $1 \mathrm{~mL}$ DMEM medium, resulting in $0.1-0.5 \%$ of DMSO, which did not affect TNBC cells (Figure S7). For testing in vivo effects of MDA-MD-231, $3 \mu \mathrm{l}$ of $10 \mathrm{mM}$ (equivalent to $186.82 \mathrm{ng}$ ) stock was added to $1 \mathrm{~mL} \mathrm{PBS}, 50 \mu \mathrm{l}$ was 
administrated directly into the xenograft grown in mouse via during day time in the animal imaging facility. For testing adverse effects of the drug, tail-vein injection was used.

Scratch (or "wound healing") assay

MDA-MB-231 cells were incubated in a 24-well plate. After reaching 70\% confluence, the scratch (or "wound") was created using a sterile $200-\mu$ l pipette tip, defined by the space within two red lines (upper left), filled (or "healed") by migration of cells (upper right). Curved red line indicates the marker (shadowed area) used to identify the location of the scratch.

Caspase-3/7 Green Fluorescence Dye

CellEvent Caspase-3/7 Green Detection Reagent was obtained from Thermo Fisher (Cat\#: C10723). Working solution of $1 \mu \mathrm{M}$ was used in cell culture. Fluorescence microscopy was used to acquire images. Green fluorescence was detected only apoptotic cells.

Cell cycle analysis using flow cytometry

Cells were grown to 60-80\% confluency in DMEM before drug treatment. Cells were either treated with BMS-191011 in DMSO, DMSO alone, or no treatment. After 24-48 hours, cells were washed with PBS and incubated with $0.25 \%$ trypsin with EDTA (Invitrogen) for 5 minutes at $37^{\circ} \mathrm{C}$. After combining the resultant solution with $10 \mathrm{~mL}$ PBS in $15 \mathrm{~mL}$ tubes, cells were pelleted at 1000 rpm for 6 minutes at $4^{\circ} \mathrm{C}$. Cell pellets were resuspended in $200 \mu \mathrm{L}$ PBS then added with swirling to $2 \mathrm{~mL}$ ice cold $70 \%$ ethanol. These single-cell suspensions were kept at $4^{\circ} \mathrm{C}$ until further processing.

For flow cytometry, cells were re-pelleted at 1000rpm for 6 minutes before decanting ethanol. After resuspension in $2 \mathrm{~mL}$ PBS and incubation for 1 minute at room temperature, cells were pelleted and resuspended in $100 \mu \mathrm{L}$ of $0.2 \%$ Tween-20 in PBS at room temperature then incubated at $37^{\circ} \mathrm{C}$ for 15 minutes. Next, $100 \mu \mathrm{L}$ of PBS containing $2 \%$ fetal bovine serum and $0.1 \%$ 
sodium azide was added and cells were pelleted. The solution was then decanted, $10 \mu \mathrm{L}$ of RNase A (DNase- and protease-free; Thermo Scientific EN0531) in PBS (180 $\mu \mathrm{g} / \mathrm{mL})$ was added and allowed to sit for 15 minutes at room temperature, and $20 \mu \mathrm{L}$ of propidium iodide (PI) in PBS $(50 \mu \mathrm{g} / \mathrm{mL})$ was added for 15 minutes. The resultant solution was brought to a volume of $300-$ $500 \mu \mathrm{L}$ using PBS before data collection.

Samples were analyzed on BD LSRFortessa using BD FACS Diva version 8.0 software. A minimum of 20,000 cells were collected for each sample. Data analysis was done using FCS Express 6.0 flow cytometry software (De Novo Software, Los Angeles CA). Cell cycle fit algorithm was selected using the lowest relative chi square value and BAD value $<20 \%$.

\section{Statistical analysis}

Data were shown as mean \pm standard deviation (SD) in the text. Bar figures were presented as mean $\pm \mathrm{SD}$ using GraphPad (Prism). Student's t-test and two-way ANOVA (for more than two groups) were used for statistical analysis. $\mathrm{P}<0.05$ was considered as statistically significant. Details in statistical analysis, such as F values and degree of freedom (DF) when using ANOVA and t values when using t-test, are included in the figure legends.

\section{Results}

1) KCNMA1 gene and protein expression in breast cancer patients

We used gene expression data (RNA-seq) from 981 breast cancer samples (TCGA Cell 2015 [23] and TCGA Provisional). In all five subtypes BK channel KCNMA1 gene expression levels are dramatically upregulated, comparison to normal breast cells (FPKM 0.7) (Figure 1). TNBC patients are represented by red dots. 
Using transcriptomics and a targeted proteomics approach, the gene-specific correlation of mRNA levels and protein copy number has been well established in human cells and tissues [24]. Previous studies have demonstrated that BK channel alpha subunit protein (main subunit forming the pore of the channel) is abundantly expressed in MDA-MB-231 cells, weakly expressed in MCF7, and nearly undetectable in normal breast epithelial cells MCF10A [15]. Therefore, we set out to investigate the protein expression of BK channels in TNBC patients' tissues.

Figure 2 shows a representative figure for protein expression of BK channel alpha subunit in primary TNBC tissue using an antibody that targets an epitope in the $1^{\text {st }}$ extracellular loop of transmembrane domains 1 and 2 (corresponding to amino acid residues 199-213 of rat KCNMA1 (Alomone Labs). MDA-MB-231 (MDA231 in the figure) was used as a positive control. Mouse brain (MB) known to express BK channels $[25,26]$ was used as an additional positive control (stronger signals in a more sensitive fluorescence image of Western blot). In addition to the glycosylated channel protein (around 200kD), there exist smaller fragments recognized by the antibody that are likely the proteolyzed C-terminals of the channel protein reported in previous studies [27]. The interpretation of the results was confirmed by incubation of the antigen (2B) that showed disappearance of the signals in $\mathbf{2 A}$. After total expression signals being normalized to $\beta$ actin, BK channel protein expression levels are nearly 14 -fold higher in primary TNBC than in normal human breast tissue (Normal: 0.345 \pm 0.177 ; TNBC: 4.793 $\pm 1.074, \mathrm{n}=4-6, \mathrm{p}<0.001)(\mathbf{2 C})$. Figure 2D shows that BK channel proteins are also abundantly expressed in different types of TNBC cells (SUM159, HCC1143), but barely detectable in MCF10A normal breast cells.

To confirm the increased protein expression levels of BK channels in TNBC patients, we performed IHC experiments in seven TNBC tissue and three normal breast tissue samples. We used a BK channel antibody that had been successfully applied in identifying KCNMA1 channel 
protein expression in the Human Protein Atlas (Sigma-Aldrich, HPA054648). Supplemental Figure 1 shows a normal breast tissue (A) and a TNBC tissue (B). The averaged percentage of protein expression area is shown in $(\mathbf{C})$. BK channel protein levels were increased by $\sim 9$-fold in TNBC than in normal breast tissue $(\mathrm{TNBC}=3.56 \pm 1.33, \mathrm{n}=7$; Normal $=0.41 \pm 0.10, \mathrm{n}=3 ; \mathrm{p}<0.01)$.

Figure 1 raised several questions: 1) Why are depolarized TNBC cells overexpressing a hyperpolarizing BK channel? 2) Are these overexpressed BK channels activated (open)? These questions led us to the hypothesis that these overexpressed BK channels are not activated. If this hypothesis is correct, then opening these channels can be exploited as a novel strategy for targeted therapy in treatment of TNBC.

For ion channels whose activity is dependent on Em, gene/protein expression levels are not intrinsically correlated with channel activity. At the resting Em of the cell, ion channels are active when they are open, inactive when they are closed. We therefore set up to investigate biophysical properties of BK channels in TNBC cells.

2) Voltage-dependent activation of BK channels in TNBC cells

Previously, we showed that the resting Em, which is within the physiological voltage range, in MDA-MB-231 cells is depolarized compared to normal mammary epithelial cells (HMEC) (Em_MDA-MB-231: about -40mV, Em_HMEC: about-67mV) [12]. To investigate whether BK channels in MDA-MB-231 are open at $-40 \mathrm{mV}$, we studied biophysical properties of BK channels in MDA-MB-231 cells using whole-cell and perforated patch clamp techniques.

Figure 3A shows the typical BK channel currents activated by the depolarizing pulse protocol (below 3A). The currents were confirmed to be generated from BK channels by iberiotoxin (IbTX, 100nM) (known as a potent specific blocker of BK channel (with $\mathrm{IC}_{50}$ of 
250pM) since it does not affect other ion channels [28]). Figure 3B shows the average voltagedependent activation curve of BK channel in eight (8) MDA-MB-231 cells. At -40mV (resting Em in MDA-MB-231), only $1 \%$ of BK channels are open.

3) BK channel opener hyperpolarizes Em in MDA-MB-231 cells

Opening large conductance (>200pS) [29] BK channels causes loss of intracellular $\mathrm{K}^{+}$ions to the outside of the cell, leading to membrane hyperpolarization. In MDA-MB-231 cells, we used

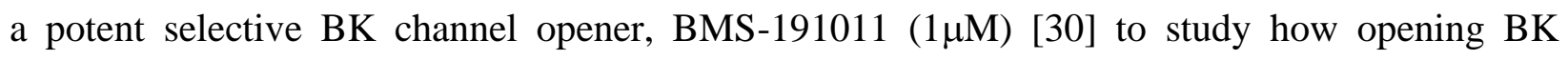
channels may affect cell Em. We found that BMS-191011 (1 $\mu \mathrm{M})$ hyperpolarized Em by $15 \mathrm{mV}$ $($ Em_con $=-30.71 \pm 8.20 \mathrm{mV}$, Em_BMS $=-45.86 \pm 8.95 \mathrm{mV}, \mathrm{n}=8 ; \mathrm{p}<0.01)$ within $15 \mathrm{~min}$ of application (Figure 3C). IbTX $(100 \mathrm{nM})$ did not induce significant change in Em (Em_con $=$ $30.71 \pm 8.20 \mathrm{mV}$, Em_IbTX $=-30.86 \pm 6.64 \mathrm{mV}, \quad \mathrm{n}=8 ; \mathrm{p}>0.05)$, but completely reversed hyperpolarization induced by BMS-191011 (Em_con $=-30.71 \pm 8.20 \mathrm{mV}$, Em_BMS+IbTX $=$ 28.43 $\pm 7.30 \mathrm{mV}, \mathrm{n}=8 ; \mathrm{p}>0.05$ ) (Figure 3C). The results provided additional evidence that the majority of BK channels are closed at Em in MDA-MB-231 cells.

4) BK channel opener induced death of human TNBC cells

To test whether hyperpolarization by opening BK channels can induce death of TNBC cells, we studied effects of BK channel opener in TNBC cell lines. Treatment of BMS - 191011 at $20 \mu \mathrm{M}$ for two days did not affect normal breast MCF10A cells (Supplemental Fig.2, left panels), but dramatically induced cell death of MDA-MB-231 cells (Supplemental Fig.2, middle panels). BMS-191011 halted growth of MCF-7 cells but induced death of much fewer cells compared to MDA-MB-231 (Supplemental Fig.2, right panels). Figure 4A shows the percentages of 
dead/dying cells are $0.92 \pm 0.29 \%(n=5)$ for MCF10A, 14.76 $\pm 1.94 \%(n=5)$ for MCF-7, and 63.82 $\pm 6.21 \%(n=5)$ for MDA-MB-231, $\mathrm{p}<0.0001$ (One-way ANOVA).

To ensure that BMS-191011 induced cell death is via opening BK channels, we used another specific BK channel opener, NS11021, which has a different chemical structure [31]. Figure 4B shows time - and concentration - dependent effects of NS11021 on the growth of MDA-

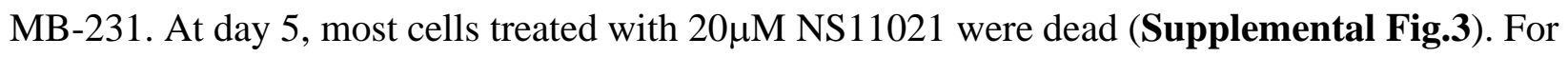
the same day, NS11021 induced cell death at different concentration is statistically significant compared to untreated group ( $\mathrm{p}<0.0001, \mathrm{n}=6)$.

To test whether BK channel opener mediated hyperpolarization-induced cell death is independent of TNBC subtypes, we studied effects of BMS-191011 on additional TNBC cell lines, SUM159 (Basal A, like MDA-MB-231) and HCC1143 (Basal B) [32]. BMS-191011 inhibited cell growth of SUM159 (4C) (and Supplemental Fig.4) and HCC1143 (4D) (and Supplemental Fig.5) cells in a similar way compared to that in MDA-MB-231. Additional controls were performed to rule out the potential side effects of DMSO on the cell growth of TNBC. Supplemental Figure 6 shows an example for 0.5\% DMSO (maximal volume used in drug treatment) that has no effect in cell death of HCC1143. We also tested that DMSO had no effects in cell growth of MDA-MB-231 and SUM159 cell lines.

Additionally, we tested whether a mutated BK channel that is permanently open (A313D), leading to hyperpolarization [33] may induce MDA-MB-231 cell death. Supplemental Figure 7 shows that after 2 days of transfection, 59.4 $\pm 13.7 \%(n=5)$ of MDA-MB-231 cells expressing A313D died, in comparison to $17.2 \pm 5.3 \%(n=5)$ of death in cells expressing wild-type (WT) channels or $18.8 \pm 7.4 \%$ of death in cells expressing on GFP plasmid $(n=5, p<0.003)$. After 4 days 
of transfection, all MDA-MB-231 cells expressing A313D were dead, whereas most cells expressing WT channels or GFP alone were alive.

\section{5) BK channel opener induced apoptosis and caspase-3 activation in TNBC}

To understand the mechanism that mediates hyperpolarization - induced death in TNBC cells, we studied apoptosis, a well-studied programmed cell death mechanism. We performed timelapse imaging experiment demonstrating that low concentration $(1 \mu \mathrm{M})$ of BMS-191011 induced rapid cell shrinkage, a distinguished event unique to apoptosis [34], within 20-60min (Supplemental Fig.8).

We also studied effect of BK channel opener in caspase activation, an established mechanism and a strong indicator of apoptosis [35]. We first used a fluorescent caspase 3/7 green dye to test whether BK channel opener may induce caspase activation in MDA-MB-231. Figure 5 shows that after 3 days of $10 \mu \mathrm{M}$ BMS-191011 treatment (5A), strong fluorescent signals (5B) were detected in MDA-MB-231. Furthermore, Figure 5C shows the presence of pro-caspase-3 protein expression in three TNBC cell lines, MDA-MB-231, HCC1143, and SUM159. BMS$191011(20 \mu \mathrm{M})$ treatment for two days induced cleaved caspase-3 expression in MDA-MB-231 (5D). These results suggested that BK channel opener can induce caspase-3 activation in MDAMB-231 cells. Thus, hyperpolarization can induce activation of caspase- 3 and apoptosis in TNBC cells.

\section{6) BK channel opener prevented migration of $M D A-M B-231$}

Majority of breast cancer patients die due to tumor metastasis and one critical step of metastasis is migration [36]. If BMS-191011 effectively induced cell death, it may prevent migration of MDA-MB-231 cells. Supplemental Figure 9 shows a typical scratch assay (or 
"wound heal") imaging experiment [37]. While control (untreated) MDA-MB-231 cells can recover from the scratch within 4 hours (upper), 100nM BMS-191011 prevented migration of MDA-MB-231 cells within 4 hours (lower). Similar results were obtained in an additional four experiments.

\section{7) BK channel opener induced arrest in cell cycle G2 phase in MDA-MB-231}

Figure 6 shows the effect of opening BK channels in MDA-MB-231 cell cycle using flow cytometry. In the absence of BMS-191011 treatment, cell distribution in G1/S/G2 phases are approximately equal (A), BMS-191011 treatment after 24h resulted in arrest in S/G2 phases associated with a decrease distribution in G1 phase (B). After 48h, cells are all arrested in G2 phase (C). As a control, we showed that DMSO (1\%) had no effects on cell cycle in MDA-MB-231 cells after 24h treatment (Supplemental Fig.10). Similar results were repeated in an additional three experiments.

8) BK channel opener induced slower growth of MDA-MB-231 xenograft in NSG mice

To test the inhibitory effects of BK channel opener on TNBC tumor in vivo, we generated MDA-MB-231 xenograft in female NSG mice at 4-week old age [21]. After a sizable tumor was formed (typically after 1-2 weeks of cell injection), BMS-191011 at $100 \mu \mathrm{g} / \mathrm{kg}$ (or $1-2 \mu \mathrm{g} / \mathrm{mouse}$ ) was directly injected into the tumor for better control of the dose and the potential loss of the drug due to rapid metabolism in mice. The drug was given twice a week in the treat group. For control group, saline was given twice a week. To avoid large variation in tumor sizes due to heterogeneity of breast cancer, we selected pairs of tumors (one as a control - injected PBS only, the other treated - injected drug in PBS) that had similar tumor sizes, and performed ultrasound imaging for four weeks to monitor effect of BK channel opener during the growth of tumors. 
Figure 7 shows a representative example for three pairs of control and treated tumors. The injection of the drug began at week5 when three pairs $(\mathrm{C} 1 / \mathrm{T} 1, \mathrm{C} 2 / \mathrm{T} 2$, and C3/T3) had similar tumor sizes, the treated tumor (T) grew significantly slower than the control tumor $(\mathrm{C})$ each week (7A). In week 8 , averaging data showed a 33\% reduction of tumor volume in drug-treated group $(\mathrm{T}=710 \pm 105, \mathrm{n}=8)$ compared to the control group $(\mathrm{C}=1056 \pm 106, \mathrm{n}=8)(\mathrm{p}<0.05)(7 \mathbf{B})$.

\section{9) BK channel opener and cardiotoxicity}

Cardiac toxicity is a major concern in anti-cancer drugs [38]. Supplemental Figure 11 shows the echocardiograph results for MDA-MB-231 xenograft mice treated with a high dose $(0.1 \mathrm{mg} / \mathrm{kg})$ of BMS-191011 compared to control (PBS treated) mice $(\mathrm{n}=3)$. There are no significant differences $(\mathrm{p}>0.05)$ between the two groups in cardiac function including heart rate, ejection fraction, left ventricular mass, and cardiac output.

Additionally, we co-cultured MDA-MB-231 with the cardiac myocytes to test the hypothesis that BK channel opener can only induce cell death in MDA-MB-231 but not in cardiac myocytes due to extremely low expression levels of KCNMA1 gene in the heart. Supplemental Figure 12 shows that BMS-191011 at $20 \mu \mathrm{M}$ indeed induced cell death only in MDA-MB-231 with little effects in cardiac myocytes after six-day incubation.

\section{Discussion}

In the present work, we showed evidence to support a hypothesis that targeted treatment by activation of BK channels - thereby hyperpolarizing the Em - can induce cell death in TNBC while sparing healthy breast cells without cardiac toxicity. We selected a BK channel opener due to overexpression of the channels in breast cancer and large conductance of the channel. Opening large conductance potassium channels can effectively induce membrane hyperpolarization due to 
rapid efflux of $\mathrm{K}^{+}$ions. Extremely low expression of BK channel gene expression in normal breast cells and cardiomyocytes ensures selective apoptosis in TNBC and absence of cardiac toxicity by BK channel opener.

Using microarrays, KCNMA1 gene expression was found to increase in several cancers including breast cancer [16]. Using RNA-seq data in a large breast cancer dataset in TCGA, we found overexpression of KCNMA1 in all subtypes of breast cancer. In principle, our approach of using BK opener for selective induction of cell death works for all subtypes of breast cancer. We focus on TNBC due to lack of targeted therapy in TNBC. It needs to be emphasized that mutations in KCNMA1 gene are rare - only 15 (7 in luminal A, 5 in luminal B, 2 in HER2, 1 in basal subtype) in breast cancer, although the gene is overexpressed and the cause of overexpression is unknown.

We verified that the BK channel alpha-subunit protein levels are significantly increased in MDA-MB-231 and in TNBC patient tissues compared to normal primary breast tissues. The overexpression of BK channels in breast cancer would intuitively lead to interest in inhibition. However, inhibition mandates that channels be active. This creates an apparent contradiction with the overexpression of an ion channel that if it were active should make the Em more negative, yet our research demonstrates a cell with a more positive (depolarized) resting Em. This contradiction can be resolved if the BK channels are not open at resting Em in TNBC. The lack of statistically significant correlation of relapse free survival rate with expression confirms the need to look beyond channel expression to channel function to answer this question of inhibition or activation.

We performed patch clamp studies and found that indeed $<1 \%$ of BK channels are open at $-40 \mathrm{mV}$, which is near the Em in MDA-MB-231 cells [12]. This result addressed the problem of a depolarized breast cancer cell overexpressing hyperpolarizing BK channels. This result also 
opened a new idea that opening BK channels in breast cancer cells should hyperpolarize Em, which may halt cell growth.

We tested this concept in three TNBC cell lines, MDA-MB-231, SUM159, and HCC1143, representing different subtypes of TNBC. We used two structurally different openers (BMS191011 and NS11021) to ensure that any effects seen in cell growth are mediated by BK channel opening. Notably, BK channel openers induced more cell death in MDA-MB-231 than in MCF-7 (a non-metastatic breast cancer cell line). One possible explanation is that BK channel protein levels are significantly higher in MDA-MB-231 than in MCF-7. Indeed, using immunofluorescence staining BK channels have been previously reported to be abundantly expressed in MDA-MB-231, very weak in MCF-7, and undetectable in MCF10A [15]. Additionally, we employed a constitutively opened mutant BK channel to demonstrate that hyperpolarization is the primary driving force to induce cell death in TNBC cells.

To explore cellular mechanisms that mediate the hyperpolarization-induced death in TNBC cells, we showed in MDA-MB-231 cells early morphology changes (shrinkage) and late activation of caspase-3 in MDA-MB-231 cells. Normotonic shrinkage of cells is a hallmark of apoptosis [34]. Caspase activation is a well-established pathway in apoptosis [39]. In addition, we showed that at low concentration (100nM), BK channel opener was able to prevent cell migration.

During cell cycle, membrane depolarization is essential for transition of G2-phase to mitosis $[9,10]$. Induced hyperpolarization during this transition can arrest cell growth by blocking DNA synthesis $[9,10]$. Indeed, our results demonstrated that hyperpolarization induced by BK channel opener caused cell cycle arrest in G2 phase in MDA-MB-231. 
In three size-matched tumor pairs, BMS-191011 slowed down the growth of xenograft tumors every week, indicating that opening BK channels in tumor can inhibit cell growth of TNBC cells in vivo. After four-week treatment, there was a statistical difference between the two groups, even using a small number of mice ( 8 per group) and twice a week drug treatment. Increasing number of mice and frequency of drug treatment may likely demonstrate a stronger statistical significance with a larger inhibitory effect in xenograft tumor growth.

A major concern in anti-cancer drugs is cardiotoxicity [38]. The KCNMA1 gene expression levels are very low in the heart (FPKM $~ 0.2$ to 0.3 ) [40]. We performed echocardiogram on the mice to assess potential cardiac side effects of BK channel opener in cardiac functions. We found no significant changes in cardiac functions in treated mice compared to untreated mice. Furthermore, in the co-culture of TNBC cells with cardiac myocytes, we showed that BK channel opener did not induce significant toxicity in cardiac myocytes, while killing TNBC cells.

Targeted therapy has significantly increased the survival rate of breast cancer patients, while cardiotoxicity remains an increased risk of cardiovascular disease (such as left ventricular dysfunction and heart failure) in breast cancer treatment [41]. For older women (>65 years) surviving breast cancer, the leading mortality is cardiovascular disease, not breast cancer itself [41].

Ion channels play a critical role in the hallmarks of cancer [42]. Gating of voltagedependent ion channels are controlled by cell membrane potential $(\mathrm{Vm})$. Opening and closing of these channels also change $\mathrm{Vm}$. For example, opening of $\mathrm{K}^{+}$channels causes membrane hyperpolarization (Vm becomes more negative) due to $\mathrm{K}^{+}$ions flowing out of the cell $[43,44]$. On the other hand, opening of $\mathrm{Ca}^{2+}$ channels increases calcium influx $\left(\left[\mathrm{Ca}^{2+}\right]_{\mathrm{o}} \sim 1-2 \mathrm{mM},\left[\mathrm{Ca}^{2+}\right]_{\mathrm{i}} \sim\right.$ 100-200nM), which contributes to membrane depolarization (Vm becomes more positive) . 
Calcium homeostasis is an extremely delicate process, disruption of calcium homeostasis triggers many pathological events including apoptosis [46, 47]. Many ion channels are overexpressed in cancer cells, but nearly undetectable in normal epithelial cells. For example, voltage-dependent calcium channels (VGCC) are readily detectable in breast cancer cells $[48,49]$, but not in human healthy mammary epithelial cells . Therefore, we demonstrated that blocking VGCC can effectively inhibit growth of breast cancer cells without affecting normal breast cells .

Recently, voltage-gated potassium channels have attracted cancer investigator for being potential targets in cancer therapy [51,52]. Kv1.3 has gained particular attention due to its low expression in the heart, while overexpressed in cancer [53,54]. We are targeting BK channel since it has the largest conductance in the potassium channel family. Opening BK channels therefore can induce the largest membrane hyperpolarization, a central strategy to breast cancer cells in which membrane potential is depolarized. BK channel expression levels in heart (RPKM $\sim 0.2$ ) is even lower than in normal breast cells (RPKM $\sim 0.7$ ), targeting BK channel activation is anticipated to have significantly less effects in cardiac functions.

\section{Conclusions}

While KCNMA1 genes are overexpressed in all types of breast cancer, targeting it is particularly useful for TNBC due to lack of effective pharmacological therapy in TNBC patients. We propose a novel approach to inhibit TNBC growth based on depolarized Em and significantly increased BK channel gene expression in TNBC. This new strategy is independent of TNBC subtypes and yields tumor-specific destruction of TNBC without cardiac toxicity.

\section{Summary}


The evidence in this research for targeted treatment of TNBC with this BK ion channel demonstrated, not only the novel discovery for treatment possibilities in TNBC, but also the significant role electrophysiology can play in granting functional insight to genetic data which translates to clinical understanding of disease and future opportunity for application. This next section reveals the circle of clinical translation when an epidemiology study shows a multi-gene signature test which could identify electrophysiology ion channel targets is not only underutilized but may contribute to why there is exponentially increased tumor growth in TNBC patients with age at diagnosis. 


\title{
CHAPTER FOUR
}

\section{Triple Negative Breast Cancer: Exponential Tumor Grade Increase with Age of Diagnosis}

\begin{abstract}
Triple negative breast cancer is an aggressive breast cancer with decreased five-year survival, increased risk for recurrence, and higher risk for metastases. Unlike other breast cancers, it has no targeted treatment and has heterogenous genetics which make classification and treatment difficult. The purpose of our research was to compare triple negative breast cancer to non-triple negative breast cancer to identify key epidemiologic factors that might lead to improved basic science directives for biomarkers, treatments, and classification. Our research uses the state cancer registry to provide the first West Virginia state-wide population evaluation of triple negative breast cancer. The research reveals novel results that tumor grade increases exponentially with the age at diagnosis. This creates an epidemiological foundation for future research to define whether the disease, access to care, biology of aging, or some other factor cause this significant finding. In addition, results reveal decreased use of testing that could be increased to improve biomarker identification, targeted treatments, and classification of triple negative breast cancer.
\end{abstract}

\section{Introduction}

World-wide, breast cancer is a leading cause of cancer mortality in women ${ }^{1}$ and in the United States, invasive breast cancer impacts $12.4 \%$ of the population of women. ${ }^{2}$ Breast cancer is a diverse disease with five molecular subtypes. One type is triple negative breast cancer (TNBC), a breast cancer where tumors are negative for estrogen receptor, progesterone receptor, and Human Epidermal Growth Factor Receptor 2 overexpression. ${ }^{3,4}$ Because of its heterogeneity, TNBC is further divided into sub-types whose classification is still controversial with the most 
recent being the genetic profile classification. ${ }^{5}$ TNBC is an aggressive disease and is associated with a poorer prognosis and 5-year survival rate as there is a higher risk for recurrence and metastasis among affected patients. ${ }^{6,7,8}$ Previous studies have shown that the prevalence of TNBC is higher in some demographic groups such as women under 40 years of age and among women of black race or Hispanic ethnicity. ${ }^{9,10,11,6,12,7,13,14}$ The reasons as to why certain demographic groups are more affected than others are unknown. Previous studies have proposed that obesity, diet, genetics, socioeconomic, and biological factors may explain differences seen among demographic groups in terms of TNBC..$^{11,6,7,15,3,16,8}$

Because of the severity of TNBC, there has been an increased interest in investigating demographic, diagnostic, and prognostic factors associated with the disease not only nationwide, but also in West Virginia.9,8,17 The age-adjusted breast cancer mortality rate among women in West Virginia is the $8^{\text {th }}$ highest among states. ${ }^{18}$ In addition to the high mortality rate, West Virginia's population is somewhat homogenous compared to other states as over $94 \%$ of the population is White Non-Hispanic; ${ }^{19}$ thus, racial differences may be observed to a lesser extent. However, the population is of lower socioeconomic status and has greater levels of obesity compared to most other states. ${ }^{20,21}$ The state also has a low net migration of residents which could indicate that hereditary factors associated with TNBC, such as BRCA mutations, may remain present in the population and influence the disease's prevalence. , $^{3,22}$

To date, two studies have investigated TNBC in West Virginia. ${ }^{8,17}$ One study found that West Virginia patients had increased representation of advanced tumors at time of diagnosis. ${ }^{17}$ The other study determined that a greater proportion of women with TNBC were under the age of 50 and that their tumors were larger than non-TNBC patients; TNBC patients also were slightly more obese than non-TNBC patients. ${ }^{8}$ However, both studies utilized unique patient populations 
from a university hospital and/or a regional medical center over 15 years ago. Thus, the purpose of this study was to investigate the demographic and diagnostic differences between those diagnosed with TNBC compared to non-TNBC utilizing more recent data from the state's cancer registry, which includes all patients diagnosed with breast cancer in the state. The findings of this study could help inform future research in a state where cancer risk and mortality are high.

\section{METHODS}

\section{Data source}

The primary data source for this analysis was the West Virginia Cancer Registry, which is maintained by the West Virginia Department of Health and Human Resources in Charleston, WV. Since 1993, the registry maintains demographic and clinical data on individuals whom are diagnosed and treated for cancer within the state. The registry also includes West Virginia residents who were treated outside the state boundary, but retain a West Virginia address. ${ }^{23}$ The registry collects, codes, and maintains these data in accordance to the National Cancer Institute's Surveillance, Epidemiology and End Results Program (SEER). ${ }^{24}$ The data are de-identified for patient confidentiality purposes.

\section{Study population}

The study population included all West Virginia women who were diagnosed with breast cancer (e.g. International Classification of Diseases for Oncology, Third Edition, codes C500-C509) from January 1, 2010 thru December 31, 2016. The data years 2010-2016 were selected because there were data quality concerns prior to 2010 and 2016 was the most recent data year available.

\section{Human subject's protections}

This study was approved by West Virginia University's Institutional Review Board (protocol \#1908679407).

\section{Variables}


The primary dependent variable was whether an individual was diagnosed with TNBC (e.g. dichotomous). TNBC was defined in accordance to SEER as a breast cancer that is negative for estrogen receptors, progesterone receptors, and Human Epidermal Growth Factor Receptor 2 overexpression. ${ }^{24}$ Various independent variables were utilized for this analysis which included: patient's age at diagnosis, race, year of diagnosis, stage of cancer at diagnosis, whether their cancer metastasized (binary), whether the patient's ipsilateral axillary lymph nodes were implicated (binary), whether the cancer was entirely insitu (binary), tumor grade, whether a multigene test was performed on the patient (binary), and whether the patient was diagnosed with Paget's disease of the breast (binary). The categorization of these variables is shown in Table 1. Race was dichotomized into white or other due the demographics of the state (e.g. it is primarily White non-Hispanic). ${ }^{19}$ For tumor grade, some patients were given a BloomRichardson score. Those with scores 3-5 were considered low grade. Those with scores 6-7 or 8-9 were categorized as moderate and high grade, respectively.

\section{Analyses}

Because the objective of this study was to compare the characteristics of women in West Virginia who were diagnosed with TNBC to other types of breast cancers, several analyses were conducted. The demographic and diagnostic characteristics between those diagnosed with and without TNBC were compared via frequencies and percentages. In order to determine which variables were associated with TNBC in patients, both binary and multivariable logistic regression analyses were conducted; these types of models were chosen because the outcome was dichotomous. ${ }^{25}$ Unadjusted (i.e. binary) models were ran between each independent variable and the outcome. All multivariable models were adjusted for age group, race, and year of diagnosis. (However, it should be noted that the multivariable model for age group was only 
adjusted for year and race and the multivariable model for race was only adjusted for year and age group). These models were adjusted for these variables because there are known differences with TNBC diagnoses among different age groups and races in other clinical populations. ${ }^{9}$ Year was also adjusted for because of the increasing awareness of TNBC in the literature overtime which could potentially influence diagnoses. ${ }^{26}$ A third set of multivariable models were ran to investigate potential effect measure modification. These models contained the same variables in the first multivariable model but also included two interaction terms; there was one interaction term between the independent variable of interest and age group and another interaction term between the independent variable of interest and race. ${ }^{27}$ All data management and statistical analyses were conducted in SAS/STAT software version 9.4 (Cary, NC) with two sided significance level $\alpha=0.05$.

\section{RESULTS}

Nearly 13\% of the women diagnosed with breast cancer in West Virginia had TNBC (Table 1).

While nearly $60 \%$ of all breast cancer patients were over 60 years of age, a slightly larger proportion of women $\leq 40$ years of age were diagnosed with TNBC (7\%) compared to those with non-TNBC (4\%). In regard to race, there was an increased percentage of non-whites (6\%) diagnosed with TNBC compared to the non-TNBC group (3\%). The stage of cancer at diagnosis confirmed a more aggressive cancer in TNBC with (4.2\%) involving direct and lymph nodes compared to non-TNBC (2.7\%) and a distant cancer in TNBC (8.1\%) compared to distant cancer in non-TNBC (5.7\%) at time of diagnosis. Metastasis was higher in TNBC (6.9\%) compared to non-TNBC (5.4\%). Tumor grade differed in TNBC patients with 73.1\% in high grade compared to only $22.6 \%$ high grade in non-TNBC. While only $26 \%$ of patients with breast cancer received a multigene test, only $6 \%$ of TNBC patients received it compared to $29 \%$ of non-TNBC patients. 
Table 2 shows the association between TNBC and demographic and diagnostic criteria. After adjusting for race and year, the odds of TNBC diagnoses in women $\leq 40$ was 2.2 times greater than the odds of TNBC in women $\geq 71$ years of age. The odds of a TNBC diagnosis in nonwhites was $71 \%$ higher than the odds of TNBC in whites after adjusting for age and year. Additionally, it appeared that TNBC was associated with higher tumor grades. The odds of TNBC was 16 times greater in high tumor grades than the odds of TNBC in women with low tumor grades at time of diagnosis after adjusting for age, race, and year. Yet, the odds of a TNBC diagnoses was $86 \%$ lower among those receiving a multigene test compared to the odds of a TNBC diagnoses among those not receiving a multigene test.

Age was an effect modifier of the relationship between TNBC diagnoses and tumor grade (Table 3). After adjusting for both year and race, the odds of TNBC dramatically increased with more severe tumor grades over the age groups. A female under the age of 40 diagnosed with TNBC had 5 times greater odds of having a high tumor grade compared to the odds of a woman in the same age group diagnosed with TNBC having a low- grade tumor. However, the odds of TNBC for a female $\geq 71$ years of age to be high grade stage was nearly 22 times greater than the odds of a TNBC diagnoses for a female in the same age group having a low-grade tumor.

Age was also an effect modifier of the relationship between TNBC diagnosis and multigene test conductance (Table 4). It appears that the conductance of the multigene test decreases with age. While there were no differences in women $\leq 40$, after adjusting for year and race, the odds of TNBC diagnoses among 41-50 year-olds is $75 \%$ lower if multigene test is conducted compared to the odds of a TNBC diagnoses among women of the same age who do not receive the multigene signature test. However, the odds of TNBC diagnoses among $\geq 71$ year-olds is $88 \%$ 
lower if multigene test is conducted compared to the odds of a TNBC diagnoses among women of the same age who do not receive the multigene signature test.

\section{DISCUSSION}

Several important findings were discovered as a result of this analysis. First, trends in TNBC diagnoses typically seen in other studies were also seen in West Virginia. Secondly, TNBC diagnosis and tumor grade varied by age, which is a novel finding. Thirdly, while multigene testing was infrequently performed among all breast cancer patients, there was an inverse relationship between age and multigene conductance among TNBC patients specifically. These are important findings especially when considering the previous literature indicating delayed diagnoses of breast cancer among West Virginia patients. ${ }^{17}$ This generates an imperative to improve early diagnosis, treatment planning, and cancer typing.

In relation to the current literature, the findings were consistent with those of previous nationwide studies. The prevalence of TNBC in West Virginia was 13\%, which is similar to the nationwide prevalence of $13 \% .^{9}$. Similarly, a there was a larger portion of patients under 40 years old within the TNBC population when compared to the non-TNBC population. Also consistent with the literature was the increased percentage of non-whites in the TNBC population despite a $94 \%$ white non-Hispanic population prevalence in West Virginia $^{19,9}$, and confirmation of TNBC's aggressive form and increased risk for metastasis. However, the majority of TNBC patients were older adults and there was a significant increased risk for high grade tumor in older patients at time of diagnoses with TNBC.

The novel finding concerning TNBC diagnosis and tumor grade variation by age provides opportunity for future research. The numbers are significant and may be due to biological reasons such as decreased physiologic response with aging, access to care, a feature of the TNBC 
disease, or some other factor. The finding warrants additional investigation, perhaps using national data.

We also found that multigene testing was not performed frequently, especially in TNBC patients, and the test's frequency decreased with patient age. The cost, difficulty of coverage for testing, and limitations of many tests in healthcare make this result unsurprising. However, the increasing benefit of genetic and epigenetic research as well as improvements to the multigene test itself may indicate opportunity for change. The data of this project indicates there may be opportunity for improved patient outcomes with future research to identify barriers to its clinical use.

New multigene signature testing provides American Joint Committee on Cancer (AJCC) staging and molecular subtyping, both valuable tools for improving our ability to classify cancer types and generate research data for identifying targeted treatment. Research by Lehman et al discovered from retrospective pretreatment biopsies that prediction regarding neoadjuvant response to therapy was not only possible but indicated the probability that there were both chemotherapeutic sensitive and chemotherapeutic insensitive subtypes in TNBC. ${ }^{28}$ In addition, research from multiple sources of basic science have linked cancer from the breast with the upregulation of a gene, protein, or general pathway. ${ }^{29}$ For example, in 2009, there was documentation of upregulation of hexokinase 2 in breast cancer brain metastasis linked with poor prognosis. ${ }^{30}$ Thus, there is indication that multigene testing could assist evaluation of tumor to aid typing and prognosis for future breast cancer patients. Multigene signature testing alone is not the complete answer to identify targeted treatment, stratify risks, and improve outcomes of clinical applications. However, improved use of this tool in conjunction with other clinical tools 
and parameters may improve patient stratification for treatment and lead to data for identifying targeted treatments.

\section{Conclusions}

Our research is broad in its evaluation by use of the entire West Virginia breast cancer registry data for our research. It is unique in its analysis to identify present evidence of TNBC disease in West Virginia and its discussion of testing practice to raise pertinent questions. It is translational in its combination of basic science and clinical application perspective.

TNBC in West Virginia is similar to the nation in its demographic evidence and its aggressive nature. Our new finding of TNBC diagnosis and tumor grade variation by age urges future research. While the discussion of Multigene signature testing reflects opportunity to evaluate policy and practice for its use. Increased efforts are being made to extend multigene testing to whole genome evaluation of tumors to bolster the information we glean from tumors. ${ }^{31}$ Therefore, increased use of the multigene signature tool may improve our ability to develop biomarkers for early identification of disease, targeted treatment, and response to therapy. Evaluation regarding our finding of exponential increase of high-grade tumor findings with increased age at diagnosis and evaluation of constraints impacting the use of the multigene signature test could be beneficial epidemiologic ventures for future research. Our research supports the need for increased focus on tumor evaluation and early diagnosis to improve outcomes for patients. 


\section{CHAPTER FIVE}

\section{Discussion and Translational Significance}

The focused interweaving of this collected work thus far allows for unique understanding of the tool of electrophysiology, its significant role in generating functional data to establish a novel target for disease treatment in $\mathrm{TNBC}$, and its translational potential to extend the circle of clinical translational science with epidemiologic evidence that clinical testing, if properly appraised and policy supported, can continue the cycle and turn breast cancer pathology into biomarkers, and ion channels into targets.

This relationship between the electrophysiologic impact of specific ion channels in resting membrane potential of the cell lines of triple negative breast cancer and the in vivo impact of treatment targeting those ion channels demonstrates the opportunity of electrophysiology to create a translational platform from basic science to clinical medicine. Clinical translational science proposes a gambit to provide research that is both basic science and clinical application in its promise. 


\section{CHAPTER SIX}

\section{Methods}

The specifics of solutions, measurements, equipment, and technique are listed in detail within the individual manuscript projects for reproducibility. This section proposes to present a broader understanding of the methods, and why they were the appropriate choice for the project. The type of research completed utilized quantitative experimental methodology for the basic science and descriptive analysis for the epidemiology. The two methods will be discussed separately.

\section{Basic Science Methods}

The experimental methodology focused on the basic science research question which investigated the apparent contradiction of overexpression of hyperpolarizing channels in a cancer cell with a membrane potential that was more depolarized. This led to the hypothesis that those overexpressed channels were not active and could be demonstrated using electrophysiology, and then exploited to terminate the cancer without harming normal cells. This would fill a knowledge

gap in the TNBC field where a targeted treatment is urgently needed. The methodological approach included the tools of electrophysiology, transfection, cell cycle analysis, in-vivo mouse modeling, and the molecular biology to evaluate resected human TNBC tumors. Experimental studies were carefully designed with representative samples and controlled variables to allow for the research to be reproducible by other researchers.

\section{Methods Terminology: Techniques of Basic Science Research}

\section{Electrophysiology}

The electrophysiology methods were designed to experimentally prove that the overexpressed BK channels in TNBC were not functioning (active/open) at physiologic voltage. The resting membrane potential for TNBC and normal breast tissue with MDA MB 231 and HMEC 
respectively is well documented and indeed TNBC is more depolarized when compared to normal breast cells; the standard resting membrane potential for normal breast cells being $60 \mathrm{mV}$ and resting membrane potential for TNBC more depolarized (positive) at $\sim-40 \mathrm{mV} .^{2,3}$ To evaluate if the channels were functioning whole cell patch clamp technique measured the resting membrane potential of TNBC by breaching the cell membrane wall, then creating a giga seal to measure its interior charge. This allowed for monitoring the voltage of the membrane potential at rest and identifying changes when BK channel openers were added. This would give us the overall $\mathrm{mV}$ of the entire cell throughout the experiment of homeostasis, BK channel opening, drug washout, and BK channel blockade. The cells resting membrane voltages were consistent with previous data revealing a depolarized cell at $\sim-40 \mathrm{mV}$. To experimentally prove the BK channels were not functioning (active/open) in this baseline recording, we then added a BK channel specific opener. If the channels were not open (active) at baseline and forced open (active) with this drug, then our patch clamp should demonstrate a more hyperpolarized (more negative) membrane potential when the drug given. The results confirmed our hypothesis and the cell demonstrated the hyperpolarized impact of 1000:1 ratio of overexpressed channels. We repeated the test at different stimulus voltages since the cell is voltage gated and the impact was consistent at each stimulus showing more BK channels functional (open/active) with the drug. 


\section{Diagram 1 BK Channels Forced Open with BK Channel Opener/Activator}

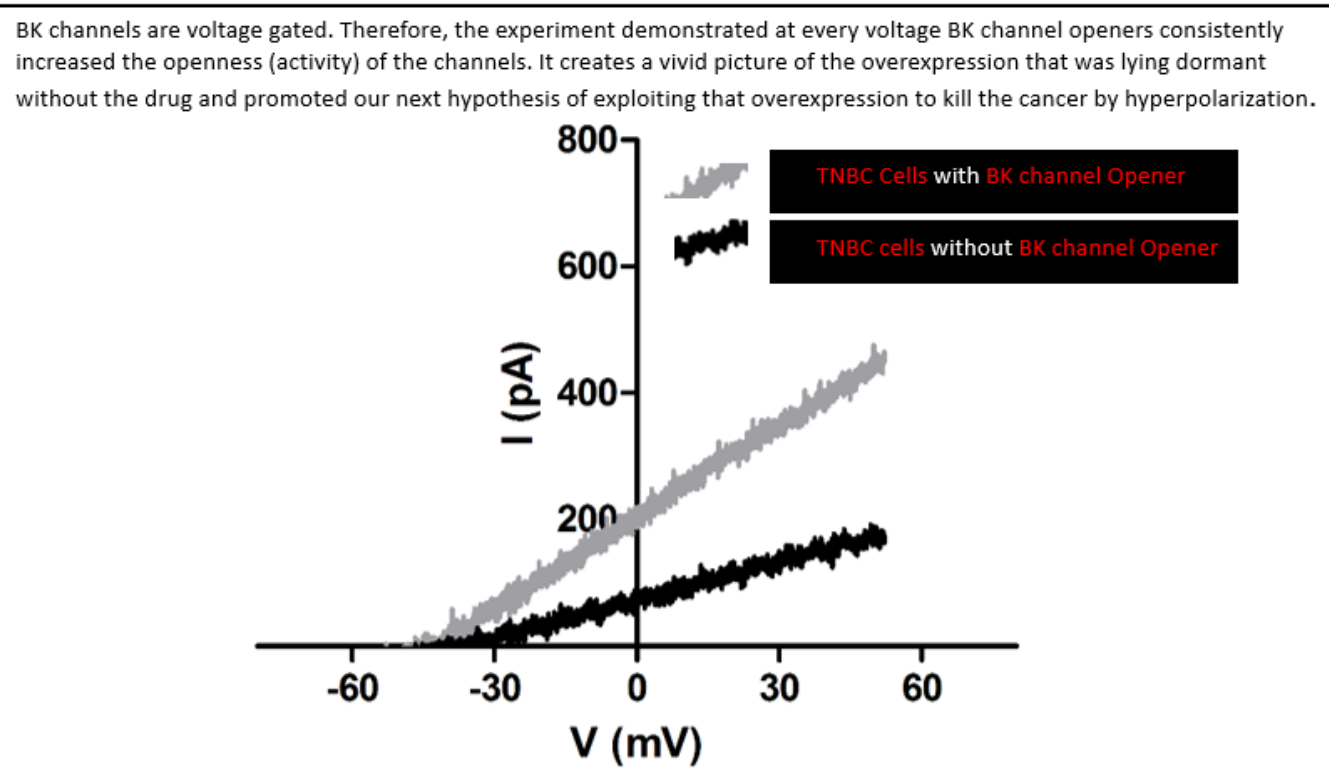

\section{Treatment In-Vitro}

These experiments provided a method of visual confirmation to our electrophysiologic data.

There are characteristic morphology changes in apoptosis seen with hyperpolarization due to loss of water following the efflux of $\mathrm{K}^{+} .4$ These experiments provided time lapse video data of the TNBC cell shrinkage with application of BK channel opener and subsequent efflux of potassium and water. In addition, experiments utilizing ethidium red confirmed significant cell death as fluorescent red circular apoptotic morphology replaced the original morphology of elongated stretched cells. Finally, the control with normal healthy breast cells demonstrated no significant death from the application of BK channel opener. These experiments were specifically designed as molecular evidence that the impact was specific to the BK channel which was overexpressed in TNBC and could be exploited to kill the cancer while not harming healthy cells which did not 
have the channels to respond to the drug. This specificity was a key component of the work to provide a targeted treatment for TNBC that would not harm healthy cells.

\section{Transfection In-Vitro (genetic mutation)}

The method of transfection in-vitro provided additional experimental evidence that the mechanism of cell death was the BK channel opening. The experiment utilized plasmid transfection of a BK channel that was mutated to always remain open thereby mimicking the impact of our BK channel opener. The result of the experiment was the same as the result with cells treated by BK channel. Significant cell death produced in mutated open BK channel TNBC but no significant cell death in wild type without mutation.

\section{Cell Cycle Changes}

Cell cycle experimental methods were designed as additional confirmation of the halt at G2 consistent with efflux of potassium from the open BK channels not allowing the cells to recover from hyperpolarization to transition into membrane depolarization for mitosis $\mathrm{M}$ phase. The experiments confirmed accumulation in G2. This method validates the hyperpolarization as mechanism. It is well known that cells hyperpolarize in G1/S and on into G2. However, it is at the G2/M border where cells must depolarize to begin the M phase. Our experimental methods showed the halting at G2 where cells were unable to recover from the hyperpolarization to enter M phase. This method design helped codify our electrophysiology data of hyperpolarization via the BK channel as the mechanism for successful targeted treatment in experiments. 


\section{Diagram 2: Cell Cycle Halted by BK channel Opener}

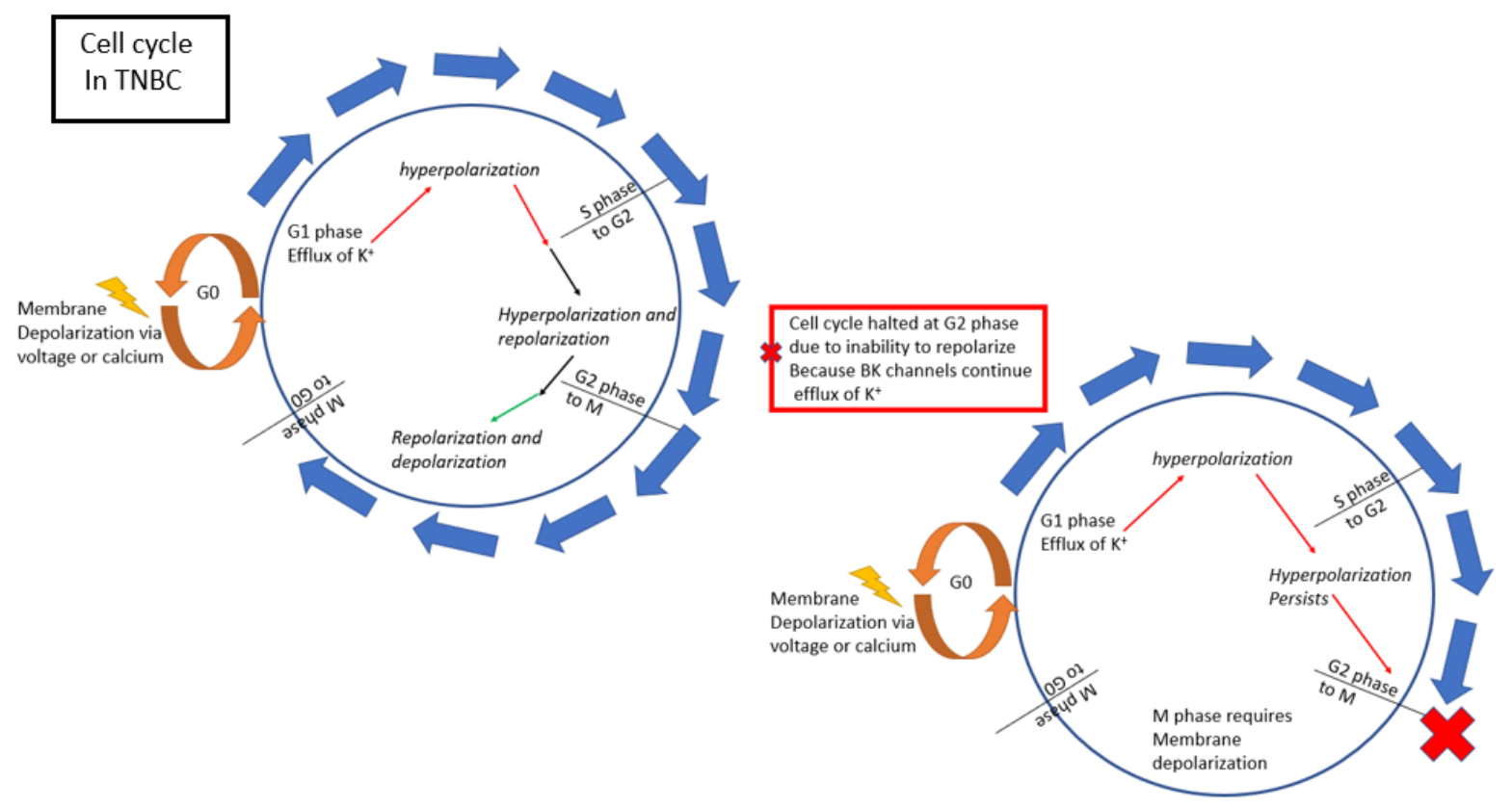

\section{In-Vivo mouse model}

The design methods for the in-vivo mouse model created systematic treatment of mice by mechanisms of tumor injection with BK channel drug to produce a treatment protocol consistent with what could be managed in a patient population. Treatments were limited to twice a week injections to better simulate patterns of treatment when considering logistics for translation to a patient population. The treatment groups were compared to control groups that were not treated with the BK channel drug. Design of methods included echocardiogram at baseline and study completion to evaluate for impact of the drug on heart function. The method design allowed for comparable analysis of tumor volume size between treatment and control groups to determine if tumor growth, a measure that has clinical significance, was different between the groups. The design not only revealed statistical significance with treatments two times a week which has clinical application in its logistical structure, but also fostered promise for future research to consider comparable experiments for more frequent treatments. 


\section{Translational Human research}

A first step considered in designing a method that created translational human research was the need for the experiments to use human resected TNBC tissue, be easily reproduced, and foundational for the work. Therefore, the logic was to use common laboratory techniques that were feasible and had long standing histories of validation as research tools. The experimental methods were then designed to compare human resected triple negative breast cancer tissue by western blot and immunohistochemistry. Western blot compared human resected TNBC tissue samples with TNBC cell lines to determine if overexpression of this BK channel was evident in human resected TNBC tissue. Immunohistochemistry provided visual evidence of the overexpression of the BK channel in the human resected TNBC tissue. These methods used validated tools with ease of reproducibility to prove BK channel overexpression is a component of the macroscopic disease of TNBC not a variant occurring due to variables such as cell line immortalization, cell passage, or other confounders. These methods create a solid foundation to build additional translational work.

\section{Epidemiology Methods}

The epidemiology methods utilized descriptive analysis of retrospective data from the West Virginia breast cancer registry. A unique tool called effect measure modification was planned in the design and methods for its benefit in identifying variables with statistical interaction. The dataset was checked for cleanliness and missing data. Then variables were transformed to streamlined datasets by clinical standards and analyzed.

\section{Effect Measure modification}

The method of effect measure modification evaluates an association measure, for example odds ratio, to determine if that measure demonstrates changes over values of some other variable. ${ }^{5}$ This is an important scientific tool when deciphering epidemiologic concerns. It allows the 
research to lay a transparency, as it were, of one variable over another and determine if there is a modification of the effect. This method exposed age at time of diagnosis as an effect measure modification. This method is a significant tool for identifying epidemiologic issues that otherwise would be overlooked. As an example, this research identified that women under forty were 5 times more likely to hear their provider say they had high grade tumor at time of diagnosis rather than low grade. With standard methodology this might get overlooked as just a feature of aggressive cancer. However, with this method we were able to identify that a patient seventy-one was 22 times more likely to hear her provider say she had high grade tumor at time of diagnosis rather than low grade. Is this a delay of care issue, significant mutation of the cancer, vulnerable population, or some other feature causing this exponential difference?

In addition, age was also an effect modifier in the multigene signature test conductance. This method exposed the underutilization of a clinical tool in a specific, high risk population. To see such significant exponential increase of high-risk tumor growth in a patient population receiving less evaluation demonstrates the value of this method for identify clinical translational opportunities to improve patient outcomes. 


\section{CHAPTER SEVEN}

\section{Literature Review}

The literature review is extensive because of the multi-disciplinary requirement of clinical translational science. The basic science covers the relevant information regarding the BK channel structure and function, the anatomy and physiology of MDA-MB-231 triple negative breast cancer (TNBC), as well as TNBC as a disease, while the epidemiology component required review of TNBC testing tools, treatment options, and how they are presently used and available. In addition, it involved defining clinical translational science and the tool of electrophysiology since neither are familiar to all scientists or clinicians.

\section{BK Channel Structure}

Structure of High-Conductance Calcium-Activated Potassium Channels (BK K $\mathrm{ca}_{\mathrm{ca}}$ ) which will be called BK throughout the rest of this manuscript is a two-unit channel with an alpha and beta unit. The alpha unit has several N-terminus isoforms which interact differently with its beta unit. ${ }^{6}$ The alpha unit is coded for by the KCNMA1 gene. ${ }^{7,8}$ There are seven components of this transmembrane unit, S0 - S6 with a C-terminus inside the cell, and an N-terminus external that reaches toward the beta unit. The $\mathrm{S} 0$ has an extra hydrophobic segment at the $\mathrm{NH}_{2}$ terminus followed by the S1 - S4 segment which holds the voltage sensitive domains, followed by the S5S6 pore domain. ${ }^{9}$ The internal terminus of the alpha unit called the C-terminus has four hydrophobic segments and alternative splicing sites. There are four more regions of the Cterminus which are a stretch of negatively charged amino acids (aspartates) called the $\mathrm{Ca}^{2+}$ bowl, a regulator conductance of potassium (RCK1). Each $\alpha-1$ contributes 2 RCK. The second regulator conductance of potassium is called the (RCK2). With each alpha contributing two RCK they form a gating ring from the 8 RCK. ${ }^{9,10}$ 
There is controversy regarding the beta subunit of the BK channel. Some research credits the beta unit with the left shift of conductance seen with different isoforms of the channel while others credit calcium as a modulator added to voltage rather than the structure of the beta unit. ${ }^{11,9}$ In addition to the controversy, there are four beta units coded by the KCNMB3 gene that function as auxiliary, $\beta 1, \beta 2, \beta 3$, and $\beta 4$ to the KCNMA alpha subunit. ${ }^{12}$ Of specific interest to clinical translational medicine is that mouse models are often used of necessity and the $\beta 3$ subunit in mice shares only $62.3 \%$ amino acid identity with humans. By contrast the, $\beta 1, \beta 2$, and $\beta 4$ have a greater consistency of $83-95 \%{ }^{12}$ Channel complexity with alternate beta subunit options that can modify an already variable alpha subunit reveals the need for functional evaluation of channels. ${ }^{11}$

The pore unit of the BK channel is part of the alpha unit. It is a hemotetramer and its identical four subunit complex demonstrates its association rather than covalent bonding between the subunits. This region is an external part of the channel and represents the area where tetraethylammonium (TEA), Iberiotoxin (IbTX), and Charybdotoxin (CTX) work when used as inhibitors of the channel. ${ }^{13,14,9}$ The turret between S5 and the pore helix of the BK channel has more residues than other potassium channels and they can reorient to impact function of the channel. ${ }^{14,15}$

The structure of the BK channel involves recognizing many facets of the channel including molecular weight and proteolytic decay. The alpha unit has 130 kilodalton $(\mathrm{kDa})$ molecular weight while the beta unit ranges from $20-30 \mathrm{kDa}$. This creates a molecular weight of $160 \mathrm{kDa}$ for the complete channel without post-translational changes or proteolytic decay. ${ }^{16}$ Early research indicates that there is discrepancy in the $\mathrm{kDa}$ molecular weight for the alpha unit in different regions of the body. This discrepancy has been shown to be the product of consistent 
and reproducible proteolytic decay from its $125-130 \mathrm{kDa}$ full length. ${ }^{17,16}$ There are distinct positions within the C-terminus of the alpha unit coded for by KCNMA1 where proteolysis occurs. This creates a product with a $90 \mathrm{kDa}, 65 \mathrm{kDa}$ polypeptide in addition to numerous smaller fragments such as $25 \mathrm{kDa} .^{17}$

\section{BK Channel Function}

There are many components to ion channel function. However, the one most relevant function for this ion channel is its role in impacting resting membrane potential. Membrane potential has three phases sometimes considered four phases; polarization, depolarization, hyperpolarization, are the three main phases while repolarization is a return to polarization state. The BK channel function in membrane potential is hyperpolarization. The hyperpolarization has different impacts depending on the tissue location of the BK channel. In neurologic systems with excitable cells the hyperpolarization can reduce firing of action potentials. In vascular tissue the BK channel function demonstrates vascular dilation ${ }^{18}$ and in non-excitable cells the hyperpolarization impacts resting membrane potential ${ }^{19}$, but little is understood about what that means for the cell. This led to our query of its role and possible target potential in triple negative breast cancer where it is overexpressed. These questions along with evidence from structure that sensitivity of the alpha unit to shifting the mid-point activation to more hyperpolarized potentials ${ }^{17}$ regardless of whether calcium or beta unit is the cause led to recognition of the knowledge gap.

Further literature review led to the vascular impact of the BK channel. "BKCa channels serve as a counter-regulatory mechanism by reverting vasoconstriction, particularly in the intense myogenic constriction of resistance vessels exposed to high intraluminal pressures." 20 This leads to a role in cardiac function and blood pressure regulation. ${ }^{21}$ 


\section{ORIGIN}

MDA MB 231 is a cell line immortalized and established in the 1970's from an original older cell line called BT20 which was established in the 1950's from a pleural effusion. ${ }^{22}$ Although this cell line was developed from a pleural effusion there is significant evidence that cell lines maintain the transcriptional and biological heterogeneity from their primary tumors. ${ }^{23,24}$ The value of cell lines persists even as new in vitro models with induced pluripotent stem cells (iPSCs) develop. MDA MB 231 was not characterized as TNBC in early years due to the lack of knowledge regarding the role of hormone receptors in breast cancer. More specific details of the maintenance of expression pattens is noted for the MDA MB 231 cell line due to its luminalbasal TNBC subtype distinction. ${ }^{24}$ There are several cell lines utilized in the research to confirm results were not in one cell line or subtype only. The HCC1143 cell line was used and it is Basal A subtype also called Basal Like. ${ }^{25}$ In addition, SUM 159 cell line was used which is similar to MDA MB 231 by subtype with both classified as Basal B or Claudin low. ${ }^{26}$ Some researchers call Basal A and Basal B lines triple negative A and triple negative B. ${ }^{26}$ The subtyping of TNBC can be confusing due to multiple names given to the same classification as well as continued controversy in typing TNBC. Newest classifications are Basal-like 1, Basal-like 2, Immunomodulatory, Mesenchymal, Mesenchymal Stem-like, and Luminal Androgen Receptor $^{27,28}$ and relate to genetic profiles for their subtype classification.

The basal-like 1 subtype has increased cell cycle and DNA damage response gene expression. ${ }^{29}$ The basal-like 2 subtype is characterized more by growth factor signaling and myoepithelial marker enrichment. ${ }^{29}$ The immunomodulatory(IM) subtype, as expected is characterized by enrichment of genes encoding for immune function such as those for immune antigens, cytokines, and core immune transduction signals. There is significant evidence that this is from 
both the tumor and infiltrating lymphocytes. The IM subtype has the largest lymphocyte percentage at 38\%. ${ }^{29,30}$ Mesenchymal has the lowest at 9\% while basal-like 2 comes in at 23\% followed by mesenchymal stem-like at $21 \%$, luminal androgen receptor at $17 \%$, and basal-like 1 at $15 \% .^{29}$

As expected, mesenchymal and mesenchymal stem-like share similarities with elevated expression of growth factor pathways. However, mesenchymal stem-like shows decreased expression of genes involved in proliferation. ${ }^{29}$

Luminal androgen receptor subtype is characterized by luminal gene expression and is predominantly propelled by the androgen receptor. ${ }^{29}$

The scientific and clinical communities have struggled to classify the subtypes of TNBC because of these genetic variations that are overlaid with relapse-free survival differences, metastatic differences, histological variation, and response to therapy variation. For example, basal-like 1 demonstrates higher grade, lower stage with increased likelihood of complete response to treatment, as well as overall increased relapse free survival. Luminal androgen receptor tumors demonstrate increased regional spread, their distant metastases is inclined to bone, and their histology, which is commonly lobular carcinoma, is exclusive to this particular subtype of TNBC. $^{29}$

Mesenchymal tumors are predisposed to metastasize to lung. Their histology is metaplastic carcinoma similar to basal-like 2, yet again is different in other capacities to warrant a separate classification of the two. ${ }^{29}$

Metabolic variation based on phosphorylation profile raised some additional molecular characterization points of consideration for classification..$^{31,32,33,34}$

\section{MORPHOLOGY}


The morphology of the MDA MB 231 cell line we used demonstrated consistent morphology in media-based incubation. The characteristic morphology of MDA MB 231 has a 200-600 $\mu \mathrm{m}^{2}$ spreading area ${ }^{35}$ in gel based culture. The cells elongate along arbitrary lines. ${ }^{36}$ These characteristics were helpful in utilization of the cells for patch clamp. The stretch allowed for examination of the cell under the microscope light to evaluate by shadow lines for the best point for an electrophysiology patch. In addition, the specific stretch and elongation pattern of the cell made the evidence of shrinkage and rounding, during treatment, as an early sign of apoptosis significantly evident.

\section{MEMBRANE POTENTIAL}

Membrane potential at the cellular level is key to this research. The electrophysiology, which is the electrical function of a normal biological organism, identifies why the overexpressed BK ion channel is not functional at physiologic voltages, and can be a potential target against TNBC.

To discuss membrane potential, it is necessary to discuss how cells mimic an electrical circuit.

\section{Diagram 3: How Cells Mimic Electrical Circuits}
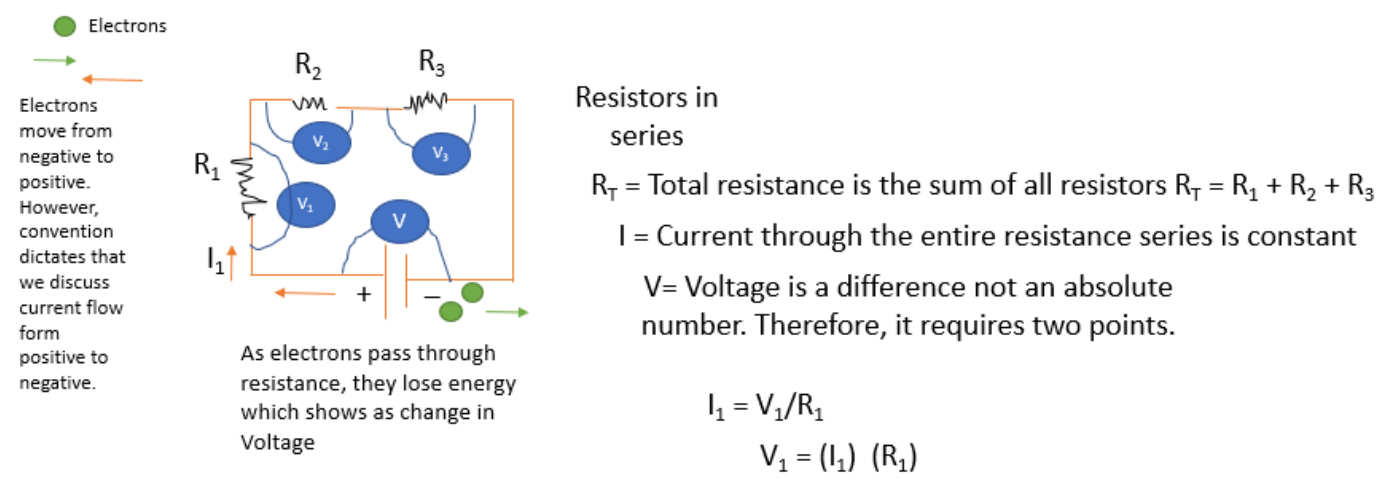


\section{Diagram 4: Understanding Voltage and Membrane Potential}

Electrical charge $+/$ - moves from inside to outside the cell or vice-versa and creates the difference which we measure as voltage.

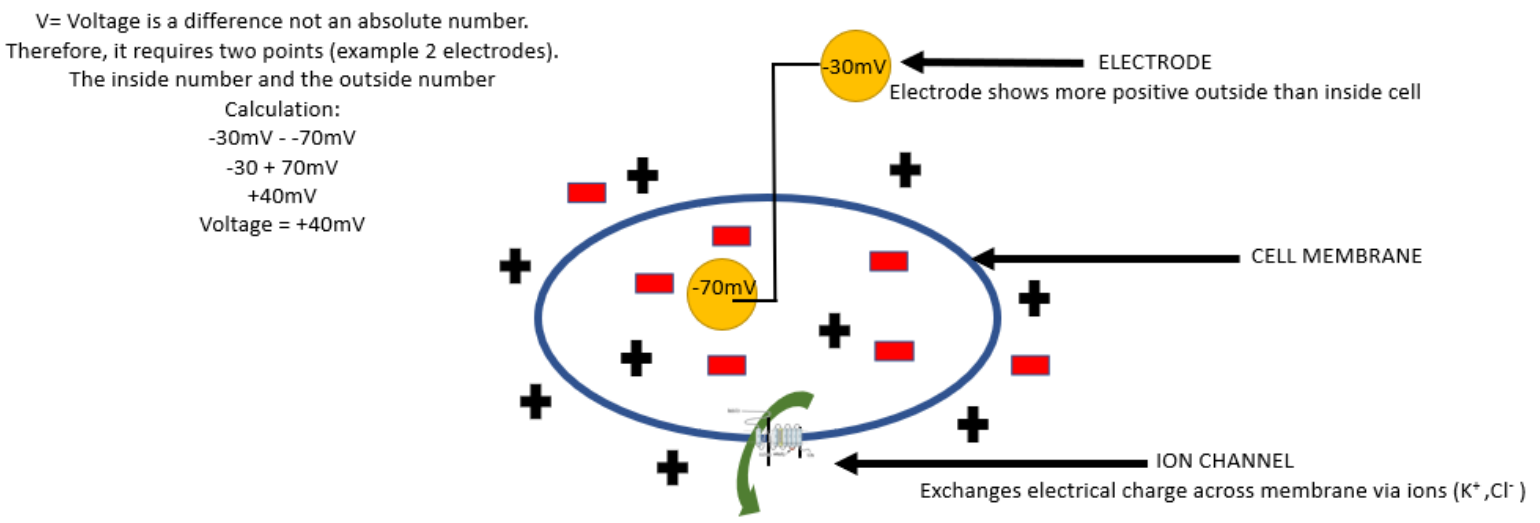

Terms:

Coulombs - The coulomb is the standard unit of electric charge. Electrons are too small to be used as the measure of electric charge. Therefore, we identify a collection of them as an electric charge. That collection is defined as the number of electrons that can pass through a set point in one second. The number of electrons that can do that is $6.24 \times 10^{18}$ electrons or one coulomb. Ampere - The ampere is the unit of electric current and is expressed as the flow of one coulomb $\left(6.24 \times 10^{18}\right.$ electrons) per second. Therefore, 1 ampere $(\mathrm{amp})=1$ coulomb per second.

In electrophysiology patch clamp measurements are in picoamps ( 1 picoamp $\left.=1 \times 10^{-12} \mathrm{amp}\right)$. Voltage - The force of potential difference. One volt is one joule of energy (work done by the force of that potential difference) per coulomb. The cell membrane potential is measured in $\mathrm{mV}$ in whole cell patch clamp. Therefore, we can measure the force of potential difference across that cell membrane and identify its quantitative number in $\mathrm{mV}$. These $\mathrm{mV}$ represent the cell potential for work (ie...joules of energy as volts). This concept is easiest to understand in a cell if 
discussed first as an action potential in a cell then discussed in regards to changes in resting potential. The Hodgkin cycle described in the original work of 1952 conveys the idea well. The opening of sodium ( $\mathrm{Na}$ ) channels moves the cell towards depolarization (more positive) by moving Na into the cell. Then as the potassium $(\mathrm{K})$ channels open $\mathrm{K}$ moves out of the cell causing hyperpolarization (more negative) membrane potential before returning to the physiologic or normal resting membrane potential. ${ }^{37}$

\section{Diagram 5: Membrane Potential: From Threshold to Hyperpolarization}

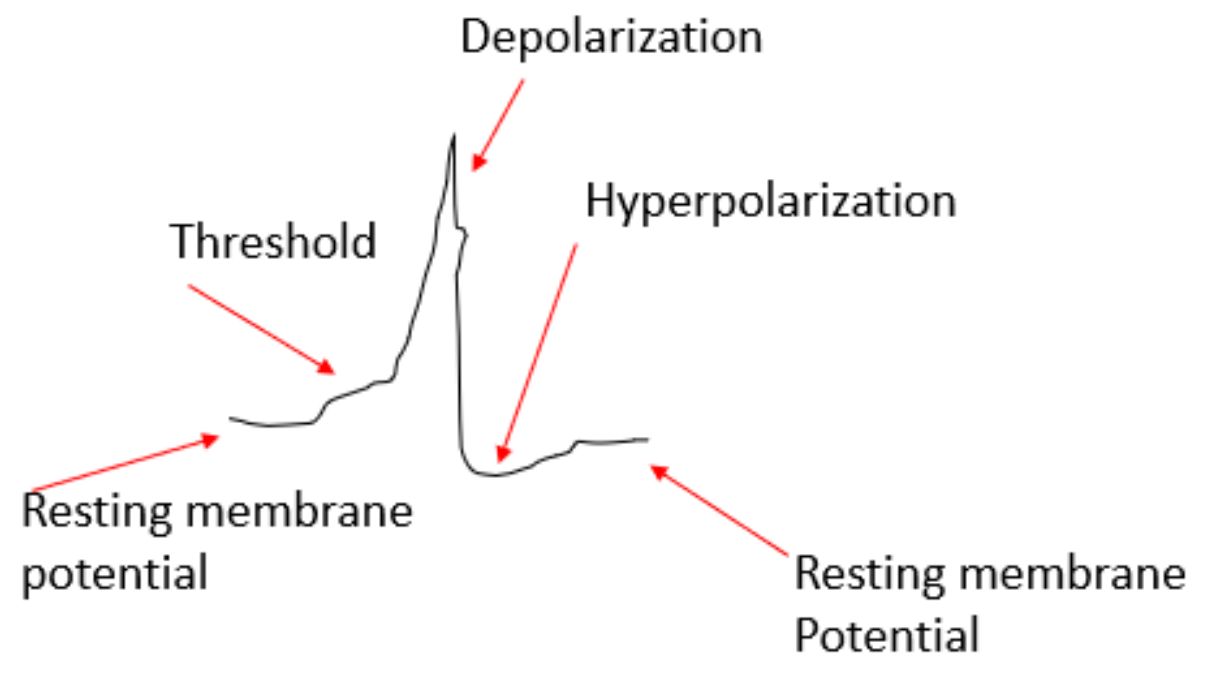

The importance of this in relation to the TNBC work is the electrophysiologic evidence that TNBC has a resting membrane potential that is more depolarized (more positive) than normal breast cells. This increased depolarization is associated with increased proliferation which is rapid reproduction of cells commonly seen in cancers. ${ }^{4}$ 


\section{Diagram 6: Resting Membrane potential TNBC vs Normal Cells}

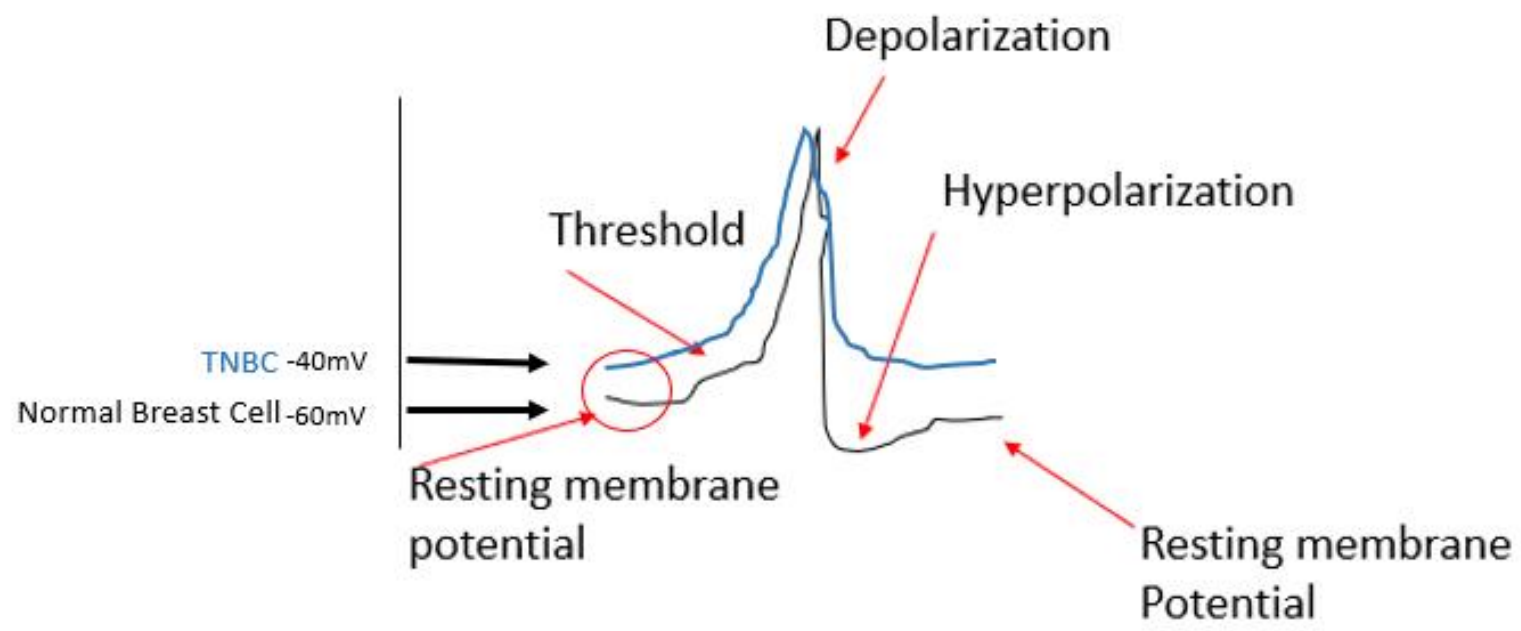

Our research confirmed this increased depolarization of the resting membrane potential of TNBC compared to normal breast cells. In addition, our novel discovery that the overexpressed BK channel in TNBC was not functioning (active) at physiologic voltage led to our hypothesis that the overexpression of the hyperpolarizing BK channel was not impacting the cell; and this could pose an opportunity to activate those channels and severely hyperpolarize and thereby kill the TNBC cells by efflux of potassium.

\section{TNBC the Disease}

TNBC is a devastating disease with a lower five-year survival rate, increased risk for recurrence, and highest rate for brain metastases of all breast cancers. ${ }^{38,39,40,41}$ TNBC is estrogen, progesterone, and human epidermal growth factor receptor (HER2) negative. This creates a significant challenge for oncologists owing to the recurrence and metastasis which result in a poor prognosis. ${ }^{42}$

TNBC Treatment Options 


\section{Chemotherapy}

Knowledge about cancer treatment for TNBC is still maturing. TNBCs are transcriptionally heterogeneous and can be grouped into subtypes with vastly differing biology and responses to chemotherapy and targeted therapies. ${ }^{43}$ Later research by Lehmann discusses the identified TNBC cell lines representative of each subtype and demonstrated differential sensitivity to alkylating agent cisplatin ${ }^{29}$ which raises new purpose for some of the testing discussed in the epidemiology project. These are treatment variations that were unknown several years ago. "In a prior retrospective analysis of patient pretreatment biopsies, TNBC type molecular subtypes were predictive of response to neoadjuvant anthracycline and cyclophosphamide followed by taxane (ACT), with BL1 subtype tumors exhibitng the highest pCR (52\%) and BL2 and LAR subtypes the lowest ( 0 and $10 \%$, respectively). These results suggest that certain TNBC subtypes are intrinsically sensitive or insensitive to neoadjuvant chemotherapy." 29 Conventional therapies employed in treatment of TNBC suffer from issues of poor bioavailability, poor cellular uptake, resistance, and undesirable off-site toxicities. ${ }^{42}$

\section{Surgery}

Surgical resection is standard care for all breast cancer. However, the decision for lumpectomy versus mastectomy often discussed with estrogen positive breast cancer patients and others is not a similar discussion with patients diagnosed with triple negative breast cancer. Although early literature up through around 2010 discusses breast conserving surgery as an option in TNBC, it is not as commonly seen after 2010 accept with adjuvant radiation therapy. ${ }^{44,45}$ The topic is controversial and many clinicians continue to recommend full mastectomy. The aggressive nature, heterogeneity, and lack of treatment options increasingly leads to mastectomy as only surgical option. Here again the increased use of clinical tests such as the improved multi-gene 
signature test could afford us valuable information about this difficult disease allowing us to create better options for care via the data produced to basic science for discovery of biomarkers, and treatment targets.

\section{Radiation}

Radiation therapy has found an increasing role in TNBC treatments with research projects that demonstrated improved five year survival and disease free rates in patients receiving radiation as neoadjuvant therapy. ${ }^{45,46}$ It is increasingly evident that radiation as neoadjuvant therapy is beneficial in TNBC patients receiving either breast conservative therapy or mastectomy and it is the molecular information regarding the biological characterization of subtypes that are improving clinician decision making for neoadjuvant therapy. ${ }^{46}$ These principles once again support the evidence of the epidemiology study that improved utilization of molecular testing such as multigene signature tests could dramatically change the landscape of treatment options and decision making in TNBC by generating the data that assists in subtyping TNBC, and identifying basic science research targets for neoadjuvant treatment in this aggressive disease.

\section{Breast Cancer Testing}

Breast cancer testing is documented across the United States by established protocols from Surveillance, Epidemiology, and End Results (SEER). SEER started in five states in 1973 and now has expanded across the United States. ${ }^{47}$ West Virginia is one of the last remaining states that do not report their data to SEER. However, the West Virginia cancer registry is set up based on the SEER protocols. The site specific cofactors listed below are from the West Virginia registry and have been placed in a table format with appropriate links for details regarding their definitions and coding. 


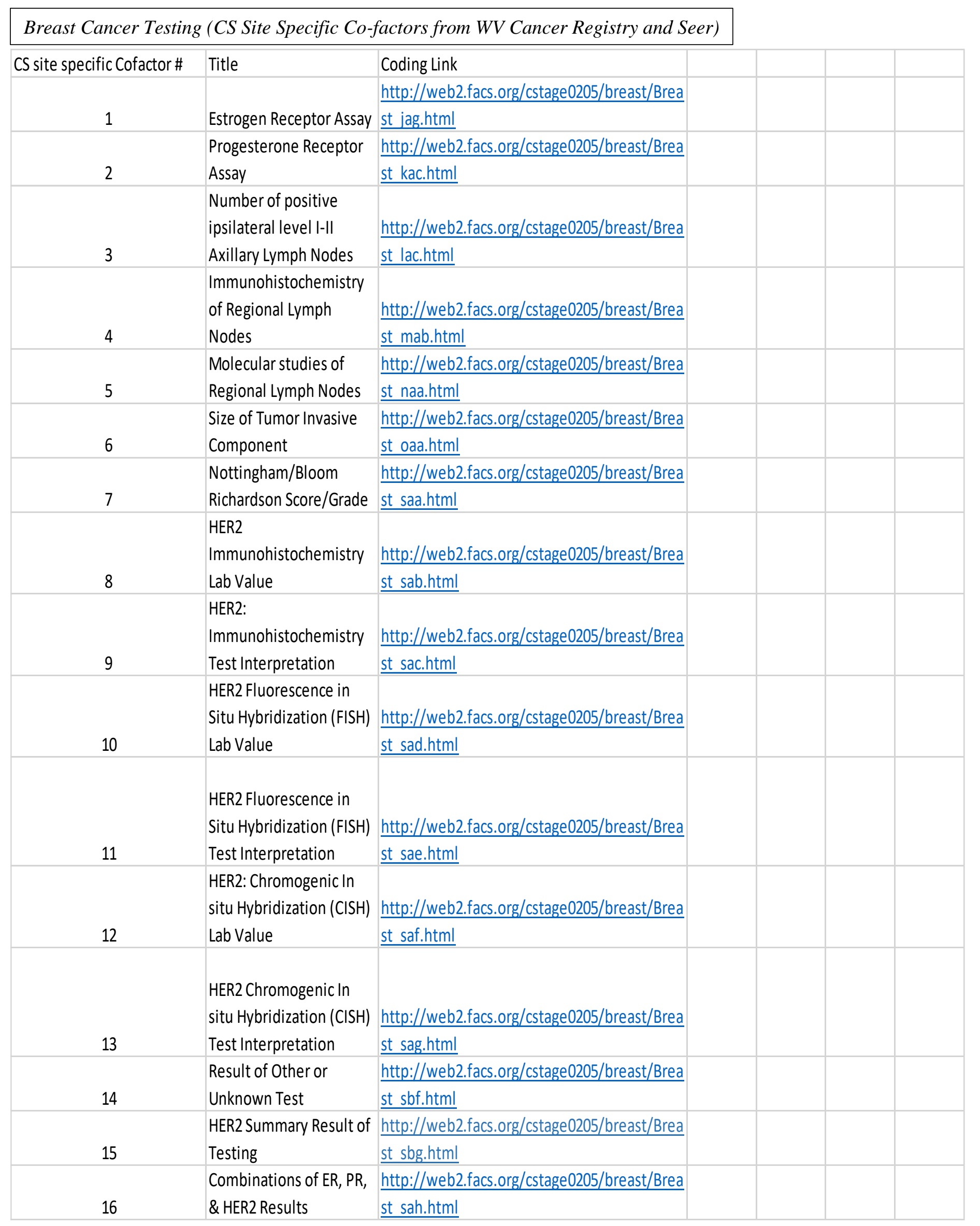




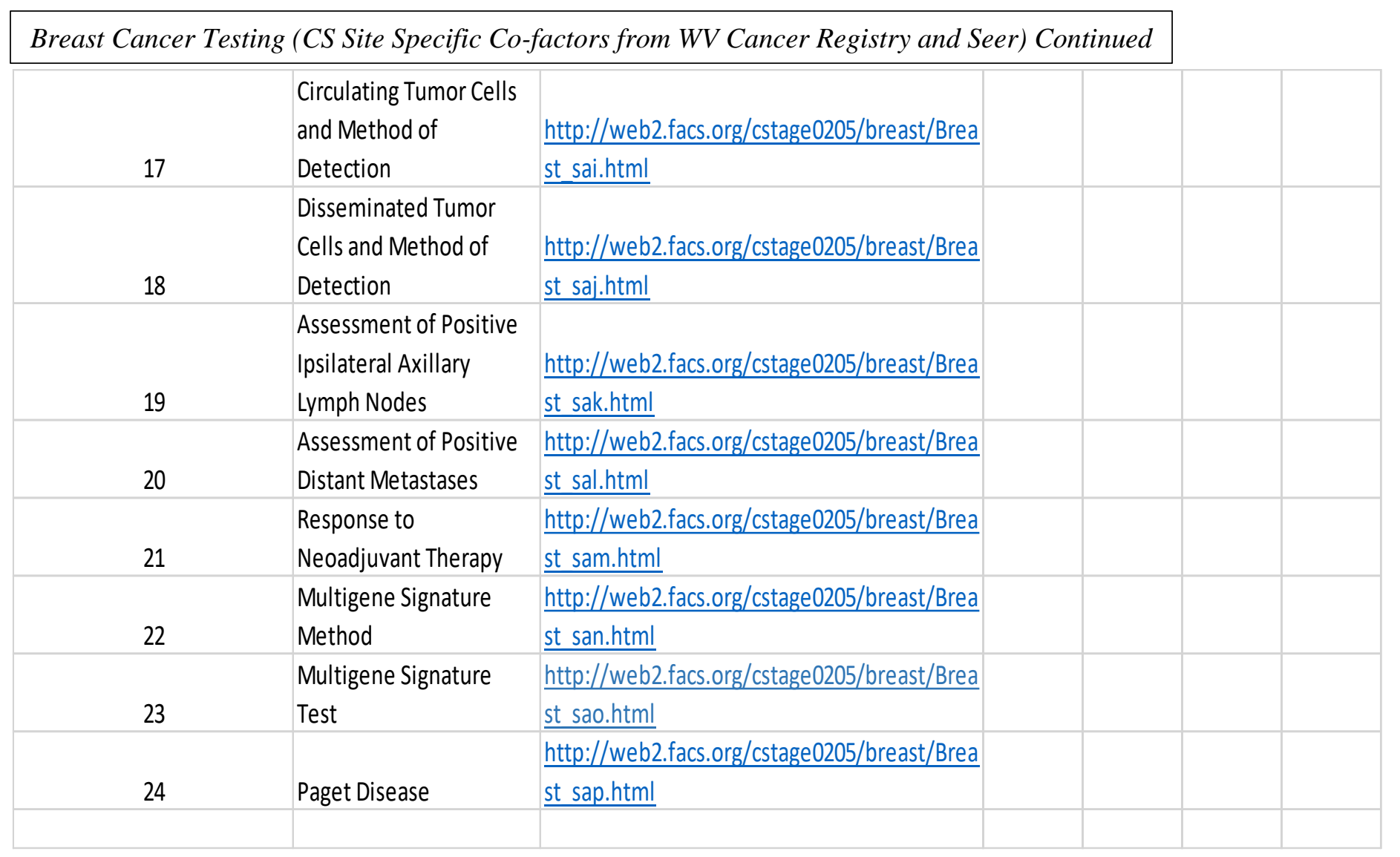




\section{CHAPTER EIGHT}

Figures and Tables

\section{Temporal Lobe Epilepsy, Stroke, and Traumatic Brain Injury: Mechanisms of Hyperpolarized, Depolarized, and Flow-Through Ion Channels Utilized as Tri-Coordinate Biomarkers of Electrophysiologic Dysfunction}
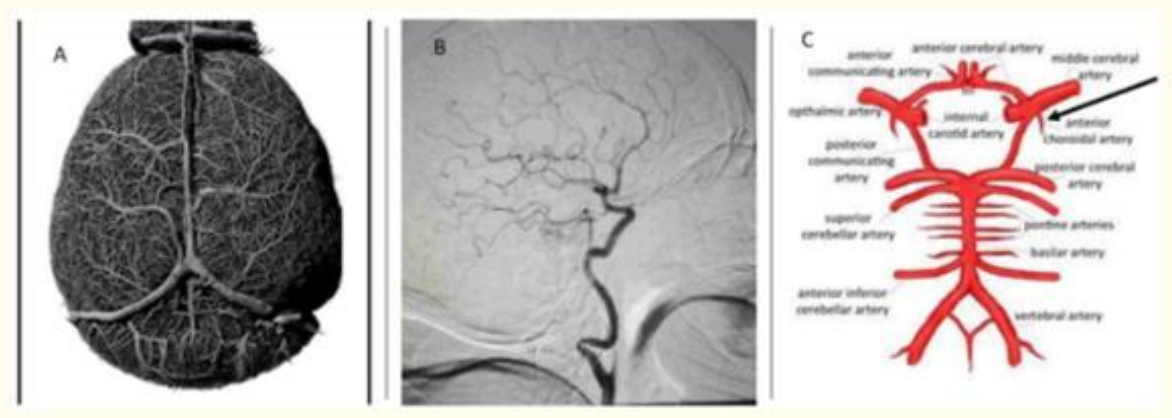

Figure 1 The casting of the entire vascular network 1A in a 24-month old male wild type mouse. The cast created with polyurethane resin and image acquired with micro CT system for visualization of collateral vasculature structure (Picture courtesy of Dominic Quintana, Neuroscience Department, West Virginia University). In 1B Computed Tomography Angiography of the normal single side internal carotid end artery system in human. The internal carotid artery supplies the anterior circulation to the brain and includes the middle cerebral artery (MCA) commonly associated with stroke and the anterior choroidal artery, which is a feeding vessel, from the anterior system, the hippocampal region associated with the sclerosis of temporal lobe epilepsy. In 1C the anterior choroidal artery in humans is the only artery branch feeding the hippocampus from the anterior system, apart from the recurrent artery of heubner. The mouse has a more collateral reach to the hippocampus possibly contributing some neuroprotection during injury. 


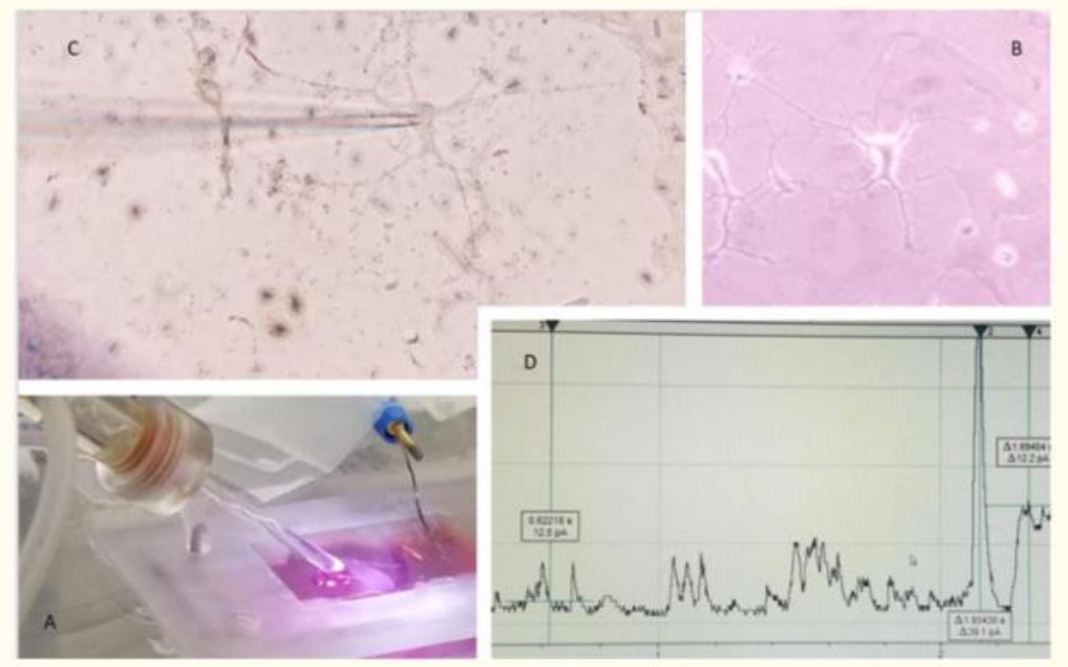

Figure 2 The glass pipette as electrode $\mathbf{2 A}$ in the recording bath where the astrocyte $\mathbf{2 B}$ is adhered to the coverslip. $2 \mathrm{C}$ demonstrates the patch between the electrode and the astrocyte under the microscope. Finally, 2D is an example of real time electrical current received from a cell.

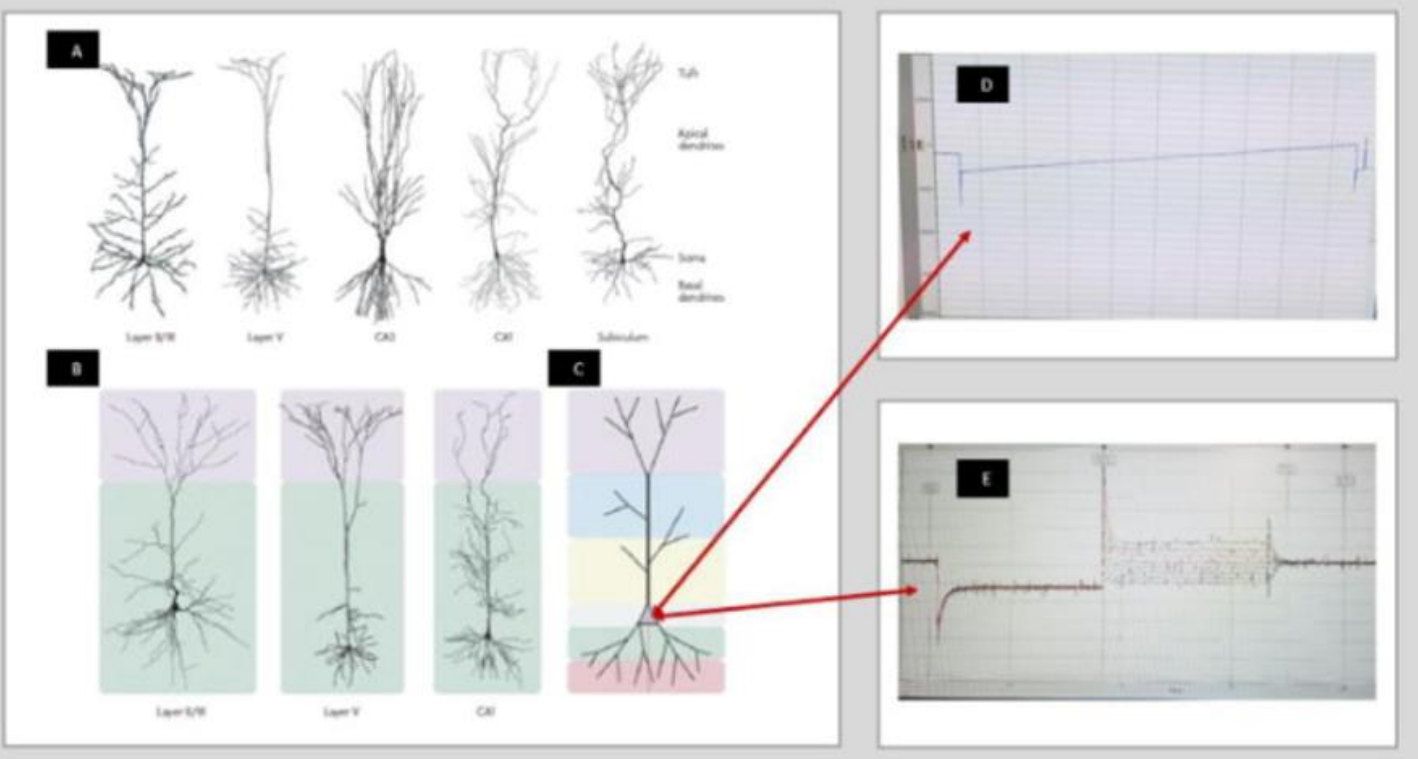

Figure 3 In 3A the variability of the pyramidal cells is shown by the CA1 and CA3 pyramidal neurons compared to the Layer V (LV) pyramidal neuron when you look at where and how the branching occurs. The LV pyramidal neuron has a streamline look and reaches through all six layers of the neocortex $3 \mathrm{~B}$ while the more compact CA1 and CA3 of the hippocampus with their 
apical branching are designed for their local purpose. In $\mathbf{3 C}$ the arrows indicate the location of the soma where patch clamp recording gives the reading of the resting membrane potential of the entire cell. Picture reprinted by permission from Macmillan Publishers Ltd: [Nature Reviews Neuroscience] [21], copyright (2008). Finally, in 3D, the ramp protocol, and 3E, the pulse protocol, you see examples of recordings received from a cell with two different protocols in the whole cell patch technique. Protocol examples are from our research lab at West Virginia University.

$$
\begin{aligned}
& E_{\mathrm{j}}=R T / n F \times \ln Q \\
& \mathrm{Cl}^{- \text {valence }}=-1 \\
& \mathrm{E}_{\mathrm{j}}=8.314 \times 298 \mathrm{~J} / \mathrm{m} \mathrm{K} /{ }^{-} 1(96,500) \mathrm{m} / \mathrm{c} \times \ln Q \\
& \mathrm{E}_{\mathrm{j}}=2,477 /-96,500 \mathrm{~J} / \mathrm{C} \times \ln Q \\
& \mathrm{E}_{\mathrm{j}}=-0.0256 \mathrm{~V} \times(2.3) \\
& \mathrm{E}_{\mathrm{j}}=-0.058 \mathrm{~V} \times \log 10[\text { [out } /[\text { in] }[120 \mathrm{mM}] /[10 \mathrm{mM}] \\
& \mathrm{E}_{\mathrm{j}}=-0.058 \mathrm{~V} \times 1.07 \\
& \mathrm{E}_{\mathrm{j}}=-0.062 \mathrm{~V} \times 1000 \\
& \mathrm{E}_{\mathrm{j}}=-62 \mathrm{mV}
\end{aligned}
$$

Figure 4 Nernst equation calculation for Chloride demonstrates the electrophysiologic alteration of membrane potential possible with fluctuations of $\mathrm{Cl}^{-}$in conditions such as ratio shift of NKCC1 to KCC2. Their correct ratio is necessary for neutrality of charge. Repeat the calculation on your own by changing the outside and inside molarity to demonstrate what can happen if the ratios of expression for these channels are not appropriate. 


\begin{tabular}{|c|c|c|c|c|c|c|c|c|c|c|}
\hline Channel & $\begin{array}{l}\text { Response to hyperpolarizing } \\
\text { voltage clamp steps at holding } \\
\text { potentials: }-\mathbf{5 3} \mathrm{mV}\end{array}$ & -60 & -67 & -74 & -81 & -88 & -95 & -102 & -109 & $\begin{array}{l}\text { Depolarization } \\
\text { To }-65 \mathrm{mV}\end{array}$ \\
\hline HCN1-- & $\begin{array}{l}\text { Ih Current Present } \\
\text { no }\end{array}$ & $\begin{array}{l}\text { Ih } \\
\text { no }\end{array}$ & $\begin{array}{l}\mathrm{I}_{\mathrm{h}} \\
\text { no }\end{array}$ & $\begin{array}{l}\mathrm{I}_{\mathrm{h}} \\
\text { no }\end{array}$ & $\begin{array}{l}\text { Ih } \\
\text { no }\end{array}$ & $\begin{array}{l}\text { Ih } \\
\text { no }\end{array}$ & $\begin{array}{l}\text { Ih } \\
\text { no }\end{array}$ & $\begin{array}{l}\mathrm{I}_{\mathrm{h}} \\
\text { no }\end{array}$ & $\begin{array}{l}\mathrm{I}_{\mathrm{h}} \\
\text { no }\end{array}$ & $\begin{array}{l}\mathrm{I}_{\mathrm{h}} \\
\text { no }\end{array}$ \\
\hline $\mathrm{HCN} 1+1+$ & $\begin{array}{l}\mathrm{I}_{\mathrm{h}} \\
\text { yes }\end{array}$ & $\begin{array}{l}\text { Ih } \\
\text { yes }\end{array}$ & $\begin{array}{l}\text { Ih } \\
\text { yes }\end{array}$ & $\begin{array}{l}\text { Ih } \\
\text { yes }\end{array}$ & $\begin{array}{l}\mathrm{I}_{\mathrm{h}} \\
\text { yes }\end{array}$ & $\begin{array}{l}\mathrm{I}_{\mathrm{h}} \\
\text { yes }\end{array}$ & $\begin{array}{l}\mathrm{I}_{\mathrm{h}} \\
\text { yes }\end{array}$ & $\begin{array}{l}\text { Ih } \\
\text { yes }\end{array}$ & $\begin{array}{l}\text { Ih } \\
\text { yes }\end{array}$ & $\begin{array}{l}\text { Ih } \\
\text { yes }\end{array}$ \\
\hline $\mathrm{HCN}_{2}-1-$ & $\begin{array}{l}\text { Ih } \\
\text { yes }\end{array}$ & $\begin{array}{l}\mathrm{I}_{\mathrm{h}} \\
\text { yes }\end{array}$ & $\begin{array}{l}\text { Ih } \\
\text { yes }\end{array}$ & $\begin{array}{l}\text { Ih } \\
\text { yes }\end{array}$ & $\begin{array}{l}\text { Ih } \\
\text { yes }\end{array}$ & $\begin{array}{l}\mathrm{I}_{\mathrm{h}} \\
\text { yes }\end{array}$ & $\begin{array}{l}\text { Ih } \\
\text { yes }\end{array}$ & $\begin{array}{l}I_{h} \\
\text { yes }\end{array}$ & $\begin{array}{l}\text { Ih } \\
\text { yes }\end{array}$ & $\begin{array}{l}\text { Ih } \\
\text { yes }\end{array}$ \\
\hline Channel & $\begin{array}{l}\text { Response to depolarizing voltage } \\
\text { clamp steps at holding potentials: } \\
-64 \mathrm{mV}\end{array}$ & -57 & -50 & -43 & -36 & -29 & -22 & -15 & - & $\begin{array}{l}\text { Repolarization } \\
\text { To }-60 \mathrm{mV}\end{array}$ \\
\hline HCN1-- & $\begin{array}{l}\mathrm{I}_{\mathrm{h}} \text { Current Present } \\
\text { yes }\end{array}$ & $\begin{array}{l}\mathrm{I}_{\mathrm{h}} \\
\text { yes }\end{array}$ & $\begin{array}{l}\text { Ih } \\
\text { yes }\end{array}$ & $\begin{array}{l}\text { Ih } \\
\text { yes }\end{array}$ & $\begin{array}{l}\mathrm{I}_{\mathrm{h}} \\
\text { yes }\end{array}$ & $\begin{array}{l}\mathrm{Ih} \\
\text { yes }\end{array}$ & $\begin{array}{l}\mathrm{Ih} \\
\text { yes }\end{array}$ & $\begin{array}{l}\text { Ih } \\
\text { yes }\end{array}$ & - & $\begin{array}{l}\mathrm{I}_{\mathrm{h}} \\
\text { yes }\end{array}$ \\
\hline $\mathrm{HCN}_{1++}+$ & $\begin{array}{l}\text { lh } \\
\text { yes }\end{array}$ & $\begin{array}{l}\text { Ih } \\
\text { yes }\end{array}$ & $\begin{array}{l}\text { Ih } \\
\text { yes }\end{array}$ & $\begin{array}{l}\text { Ih } \\
\text { yes }\end{array}$ & $\begin{array}{l}\mathrm{Ih} \\
\text { yes }\end{array}$ & $\begin{array}{l}\mathrm{l} h \\
\text { yes }\end{array}$ & $\begin{array}{l}\text { lh } \\
\text { yes }\end{array}$ & $\begin{array}{l}\text { Ih } \\
\text { yes }\end{array}$ & - & $\begin{array}{l}\text { Ih } \\
\text { yes }\end{array}$ \\
\hline $\mathrm{HCN}_{2}--$ & $\begin{array}{l}\mathrm{I}_{\mathrm{h}} \\
\text { yes }\end{array}$ & $\begin{array}{l}I_{h} \\
\text { yes }\end{array}$ & $\begin{array}{l}\text { Ih } \\
\text { yes }\end{array}$ & $\begin{array}{l}\text { Ih } \\
\text { yes }\end{array}$ & $\begin{array}{l}\text { Ih } \\
\text { yes }\end{array}$ & $\begin{array}{l}\mathrm{I}_{\mathrm{h}} \\
\text { yes }\end{array}$ & $\begin{array}{l}\mathrm{I}_{\mathrm{h}} \\
\text { yes }\end{array}$ & $\begin{array}{l}\text { Ih } \\
\text { yes }\end{array}$ & - & $\begin{array}{l}\text { Ih } \\
\text { yes }\end{array}$ \\
\hline
\end{tabular}

Table 1 The voltage measurements from Della Santina [44] in their work with HCN1 channels in the retina converted to a table to demonstrate the value of monitoring $\mathrm{I}_{\mathrm{h}}$ current of the $\mathrm{HCN} 1$ channel. The results indicate the channel in response to hyperpolarization and depolarization and indicate the potential for biomarker mechanism in the electrophysiologic function of neurologic disease. 
Opening Large-Conductance Potassium Channels Selectively Induced Cell Death of TripleNegative Breast Cancer

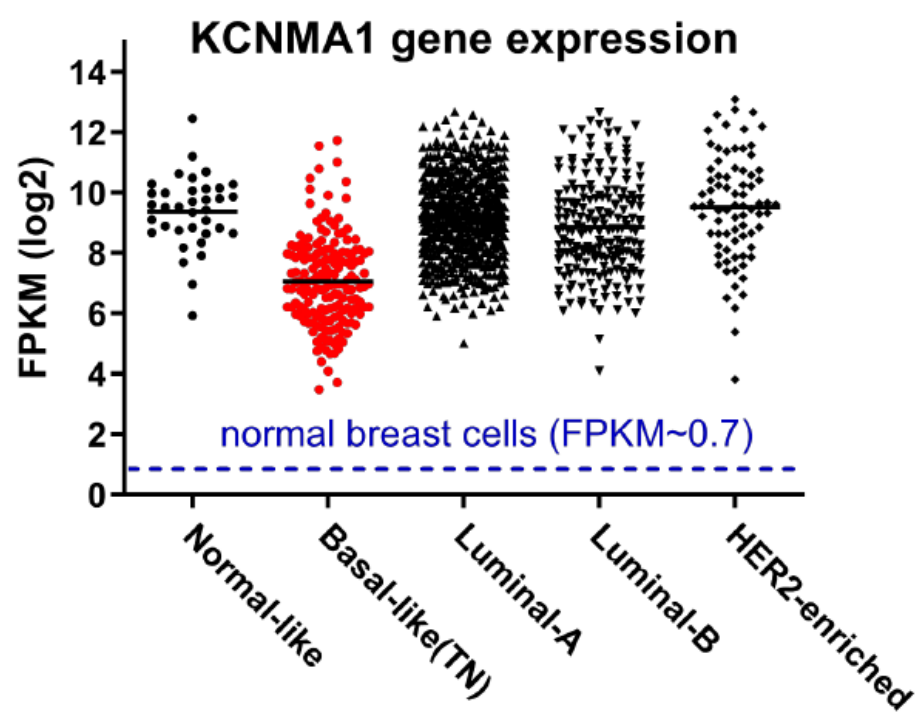

Figure 1 KCNMA1 gene expression in five molecular subtypes of breast cancer. Each dot represents one patient. Gene expression levels are presented as FPKM in log2 expression. Red dots are basal-like triple-negative (TN) breast cancer patients. Blue dotted line indicates KCNMA1 gene expression in normal breast cells. FPKM: Fragments Per Kilobase of transcript per Million. 

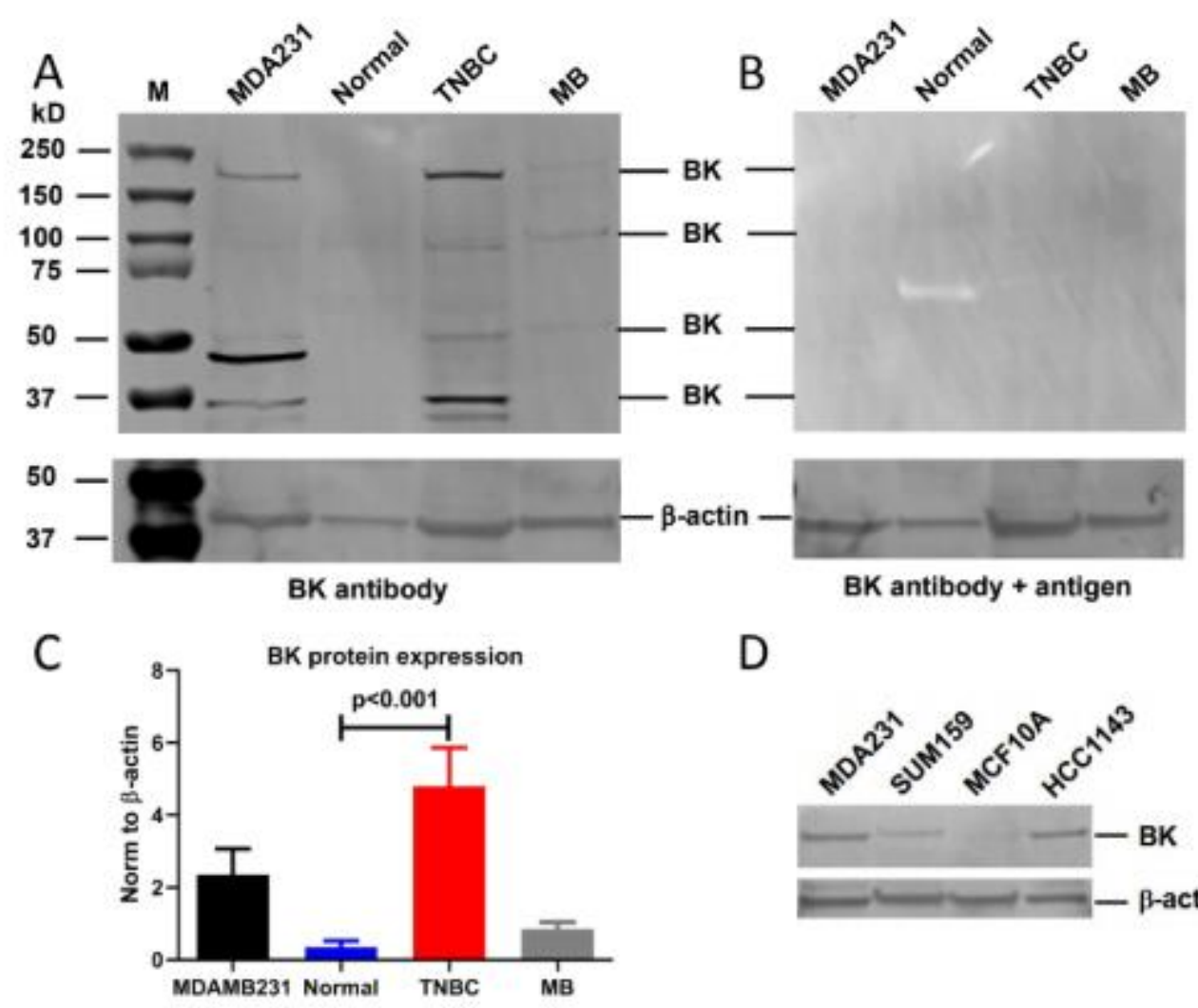

D

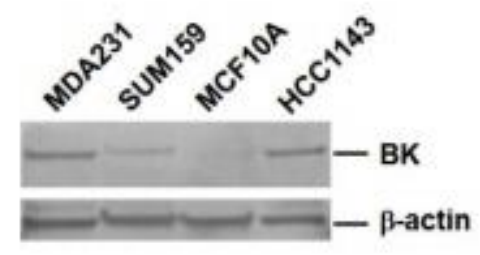

Figure 2 Protein expression of BK channel $\alpha$-subunit (forming the functional channel) in TNBC cells and patient tissues. (A) Western blots with antibody, M: marker, MDA231: MDAMB-231 cells (cell positive control), Normal: normal primary breast tissue, TNBC: TNBC patient tissue, MB: mouse brain (tissue positive control). $\beta$-actin was used as a loading control. (B) Western blots with antibody and antigen, confirming signals detected by the antibody in (A). (B) Quantitative expression levels of BK channel proteins normalized to $\beta$-actin $(n=4-6)$. Unpaired $t$ test was performed. Two-tailed $\mathrm{p}=0.0002, \mathrm{t}=0.8172$. (D) Western blots using a $\mathrm{BK}$ channel protein antibody in MDA231, SUM159, MCF10A, and HCC1143, b-actin as loading controls. 

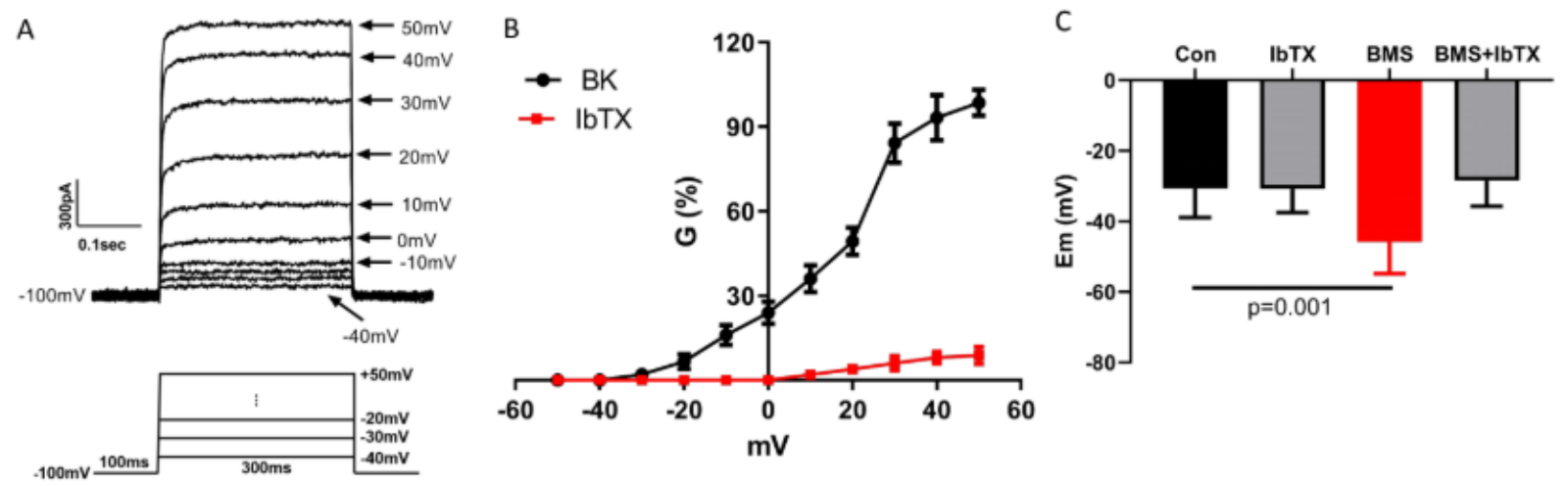

Figure 3 Biophysical properties of BK channels in MDA-MB-231 cells. (A) BK channel currents elicited by depolarizing pulses ranging from $-40 \mathrm{mV}$ to $+50 \mathrm{mV}$ in $10 \mathrm{mV}$ increment, pulse protocol is shown below. (B) BK channel conductance, $\mathrm{G},-$ voltage relationship in the absence (black) and presence (red) of $0.1 \mu \mathrm{M} \operatorname{IbTX}(\mathrm{n}=8)$. (C) Effects of BMS-191011 and IbTX on membrane potential (Em) in MDA-MB-231. 

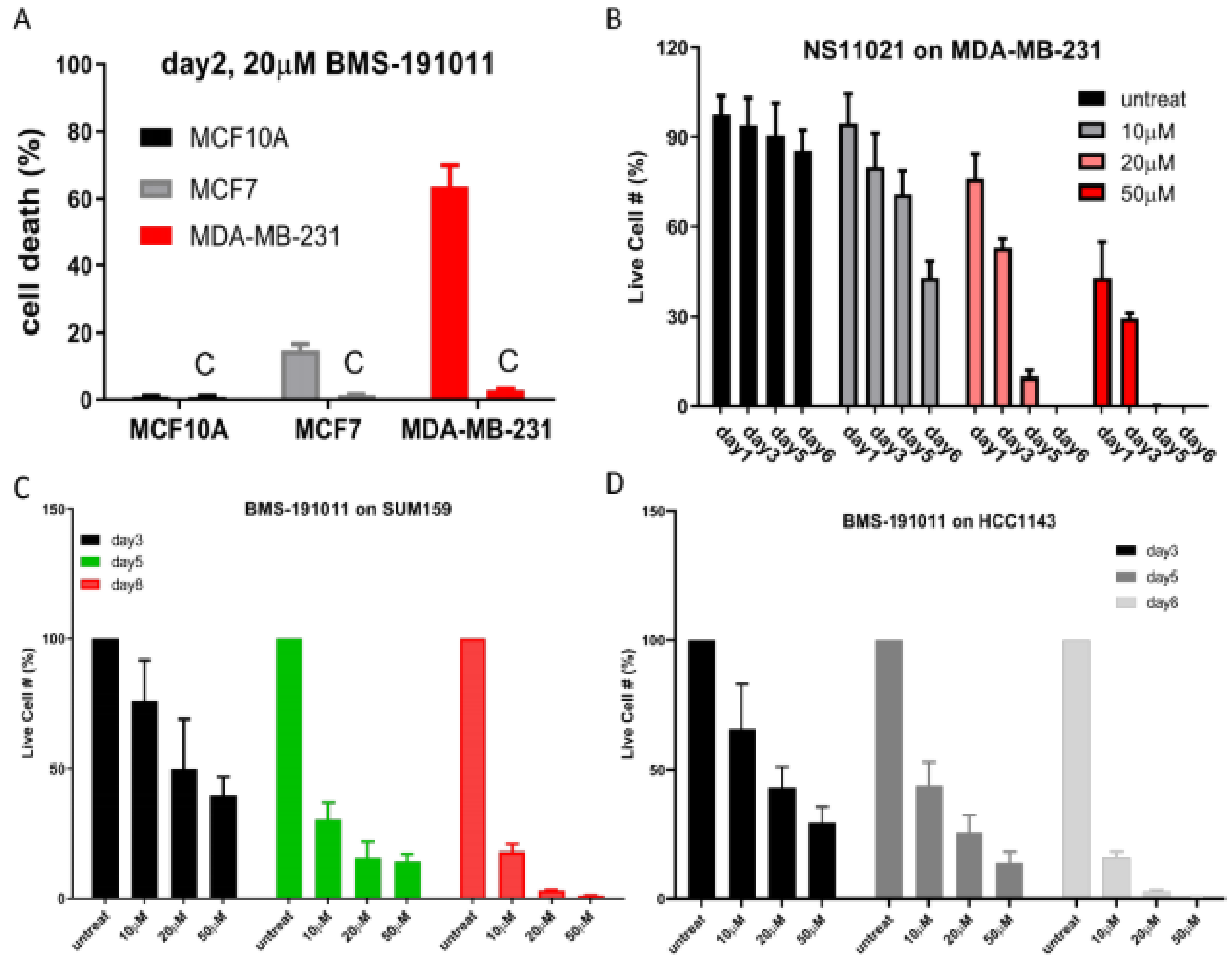

Figure 4 BK channel openers induced cell death in TNBC cell lines. (A) BMS-191011 (20 $\mu \mathrm{M}$ ) induced cell death on normal breast cells (MCF10A, dark), ER+ breast cancer cells (MCF7, grey), and TNBC cells (MDA-MB-231, red) (n=5). One-way ANOVA was performed, $\mathrm{p}<0.0001, \mathrm{~F}=65.38$. (B) Time- and concentration-dependent effects of NS11021 on growth of MDA-MB-231 cells ( $n=6)$. Two-way ANOVA was performed, $p<0.0001, F=66.67, D F=9$. (B) Time-dependent effect of BMS-191011 $(20 \mu \mathrm{M})$ on growth of SUM159 cells $(n=4)$. Two-way ANOVA was performed, $\mathrm{p}<0.0001, \mathrm{~F}=618.9, \mathrm{DF}=6$. (D) Time-dependent effect of BMS-191011 $(20 \mu \mathrm{M})$ on HCC1143 cells $(\mathrm{n}=4)$. Two-way ANOVA was performed, $\mathrm{p}<0.0001, \mathrm{~F}=27.6, \mathrm{DF}=4$. 


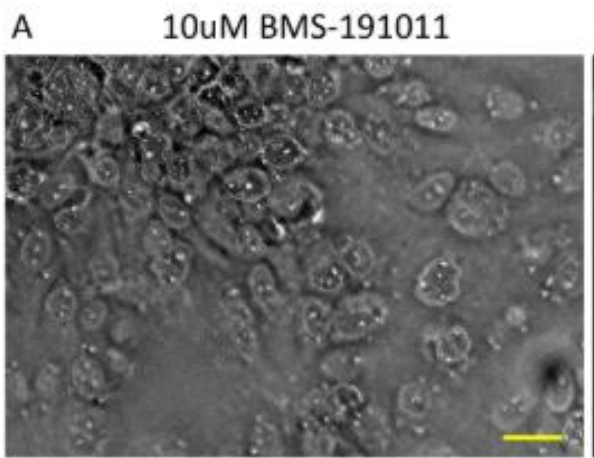

B 1 uM caspase $3 / 7$ green dye

C

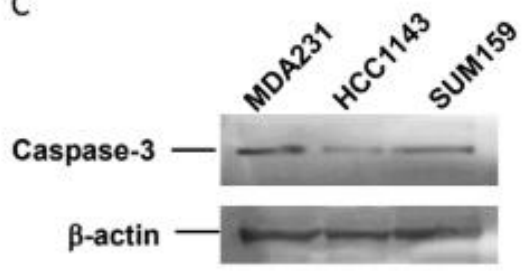

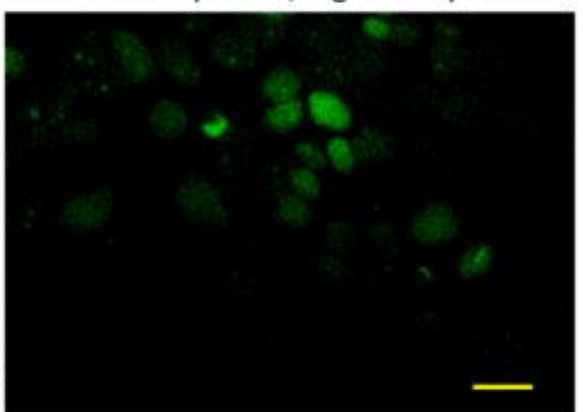

D
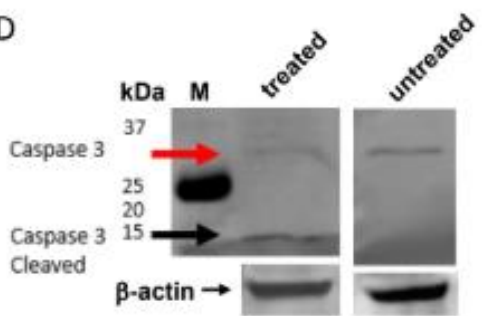

Figure 5 BMS-191011 induced activation of caspase-3 in MDA-MB-231. Using caspase-3/7 green dye, fluorescence was detected after incubation of $10 \mu \mathrm{M}$ BMS-191011 for three days (B). Light image is shown in (A). Scale bar: $20 \mu \mathrm{m}$. Using immunoblots, caspase-3 was detected in three TNBC cell lines (MDA-MB-231, HCC1143, SUM159) (C). Cleaved caspase-3 was detected after BMS-191011 treatment (D, M-marker, red arrow indicates pro-caspase 3, black arrow indicates cleaved active caspase 3 ).
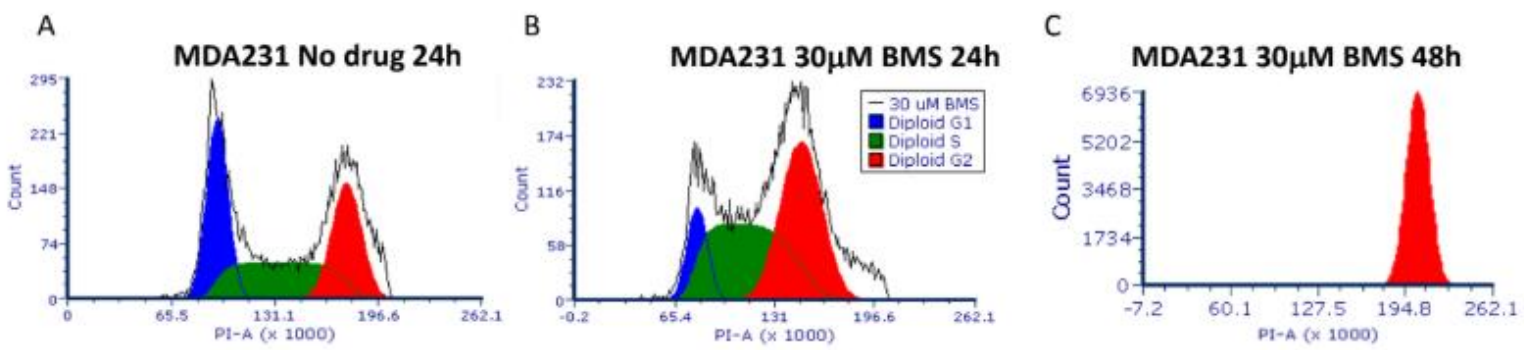

Figure 6 BMS-191011 induced arrest in S/G2 phases of MDA-MB-231. (A): No treatment (control), (B): Drug treatment for 24h, (C): Drug treatment for 48h. Blue: G1 phase; Green: S phase; Red: G2 phase. Count: amount of cells, PI-A: fluorescence intensity. 

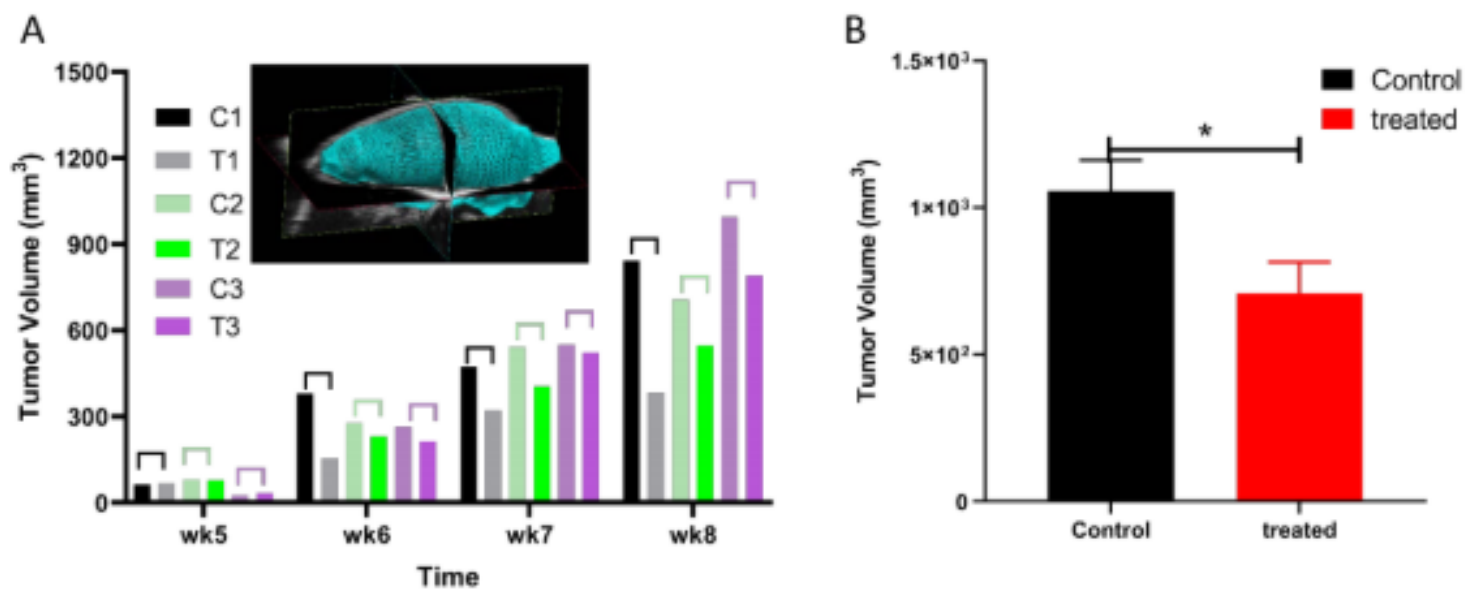

Figure 7 Slowing of MDA-MB-231 xenograft tumor by BK channel opener. (A): Three paired-tumors of similar sizes $(\mathrm{C} 1 / \mathrm{T} 1, \mathrm{C} 2 / \mathrm{T} 2, \mathrm{C} 3 / \mathrm{T} 3)$ are shown to illustrate the inhibitory effects of BMS-191011 in tumor growth. Ultrasound imaging was performed once a week to calculate the tumor volume $\left(\mathrm{mm}^{3}\right)$ for four weeks. A reconstruction of the tumor volume from ultrasound imaging is shown in the inset. (B): Average tumor volume in control $(n=8)$ and treated groups $(\mathrm{n}=8) . * \mathrm{p}<0.05$ ( $\mathrm{t}$-Test was performed, two-tailed $\mathrm{p}=0.0356, \mathrm{t}=2.32592)$. 


\section{SUPPLEMENTAL FIGURES}

\section{Supplemental Fig. 1}
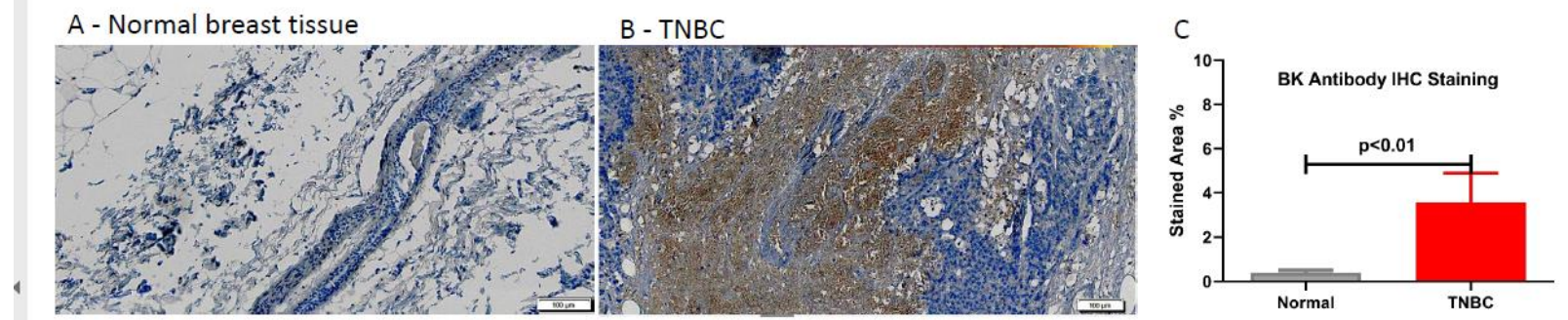

Supplemental Fig. 1 Immunohistochemistry of BK channels in TNBC patient tissues (IHC). (a) normal breast tissue, (b) TNBC tissue (BK channel expression indicated by brown color, see Methods), (c) Percentage of staining area averaged from seven TNBC and three normal breast tissues. Unpaired t-test was performed, two-tailed $\mathrm{p}=0.0042, \mathrm{t}=3.95$.

\section{Supplemental Fig. 2}

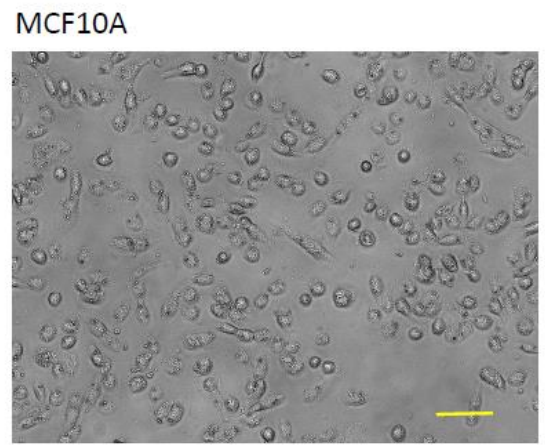

MDA-MB-231
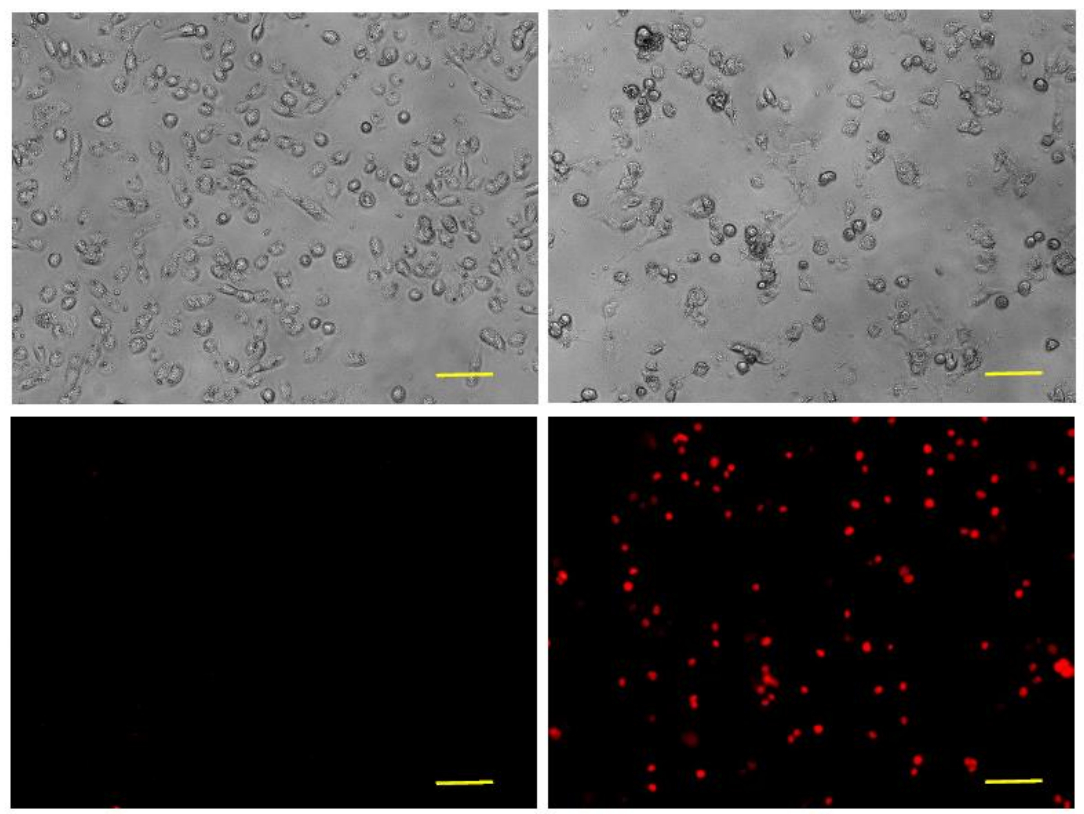

MCF7
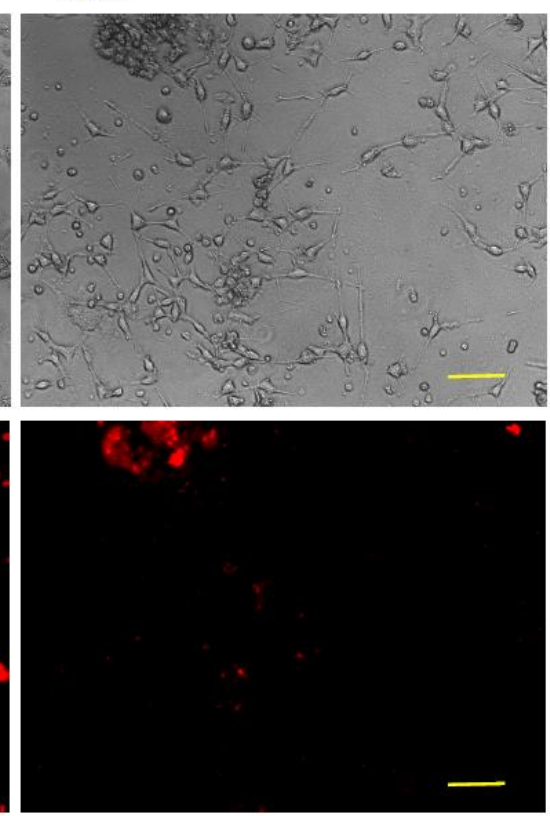

Supplemental Fig. 2 BMS-191011 on human breast cancer cell lines. Upper panels show the bright-field images, lower panels show the dead cells labelled by EthD1 dye (red). BMS-191011 (20 $\square$ M) treatment of MCF10A (left), MDA-MB-231 (middle), and MCF7 (right) after 2 days. Scale bar: $20 \square \mathrm{m}$. 


\section{Supplemental Fig. 3}

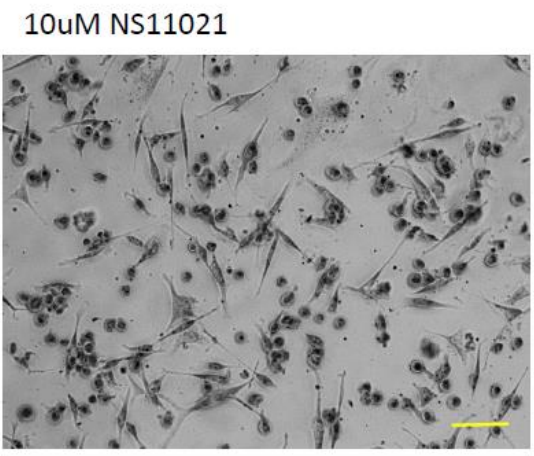

20uM NS11021
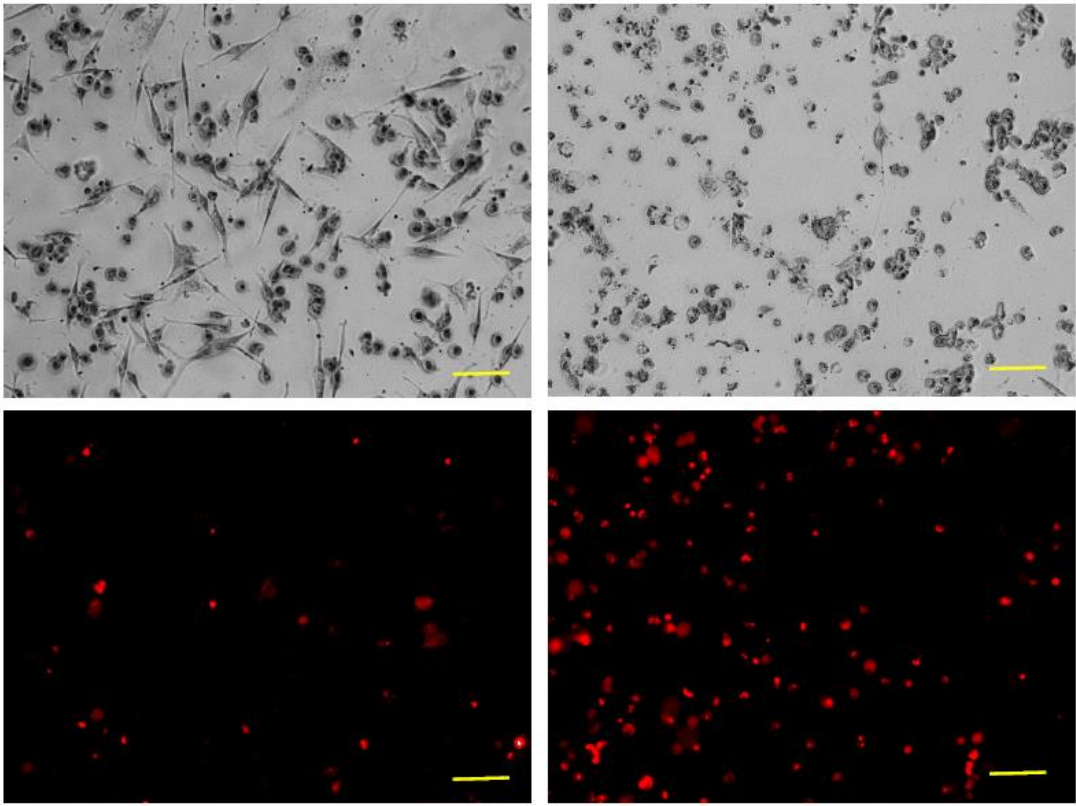

50uM NS11021
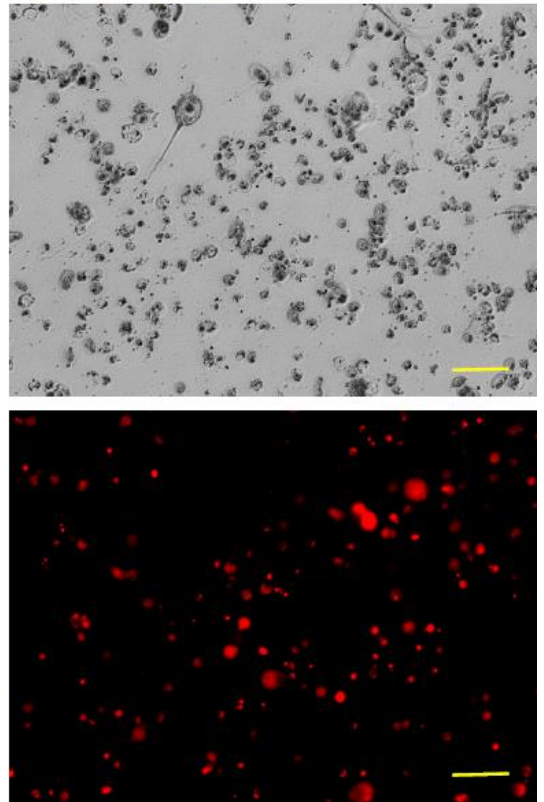

Supplemental Fig. 3 Concentration-dependent effects of NS11021 on MDA-MB231 after 5 days. Upper panels show the effects of NS11021 in bright field, lower panels show the dead cells labelled by EthD-1 dye (red). Scale bar: $20 \square \mathrm{m}$.

\section{Supplemental Fig. 4}
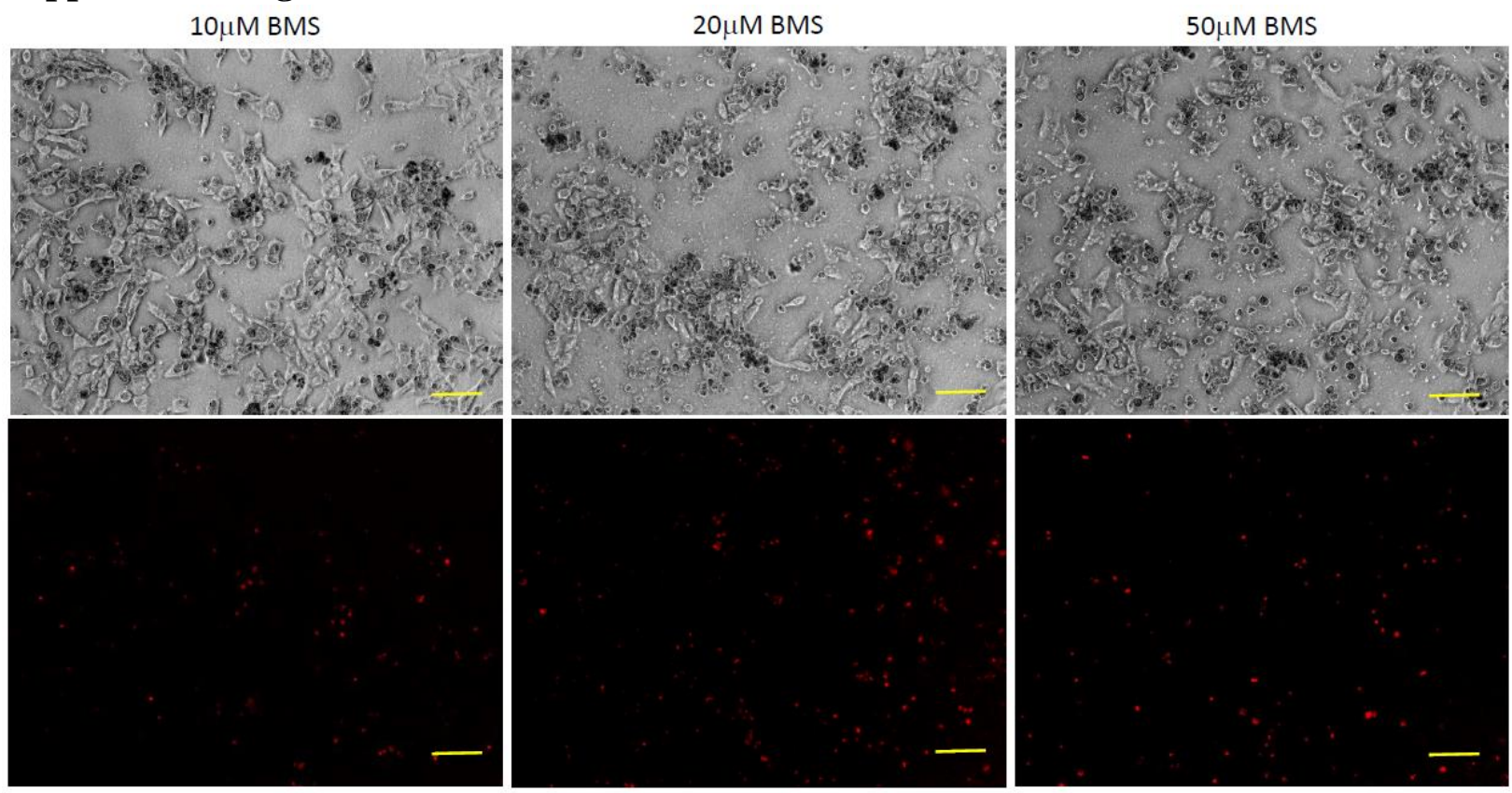
Supplemental Fig. 4 Concentration-dependent effects of BMS-191011 on

SUM159 after 5 days. Upper panels show the bright-field images, lower panels show the dead cells labelled by EthD-1 dye (red). Scale bar: $20 \square \mathrm{m}$.

\section{Supplemental Fig. 5}

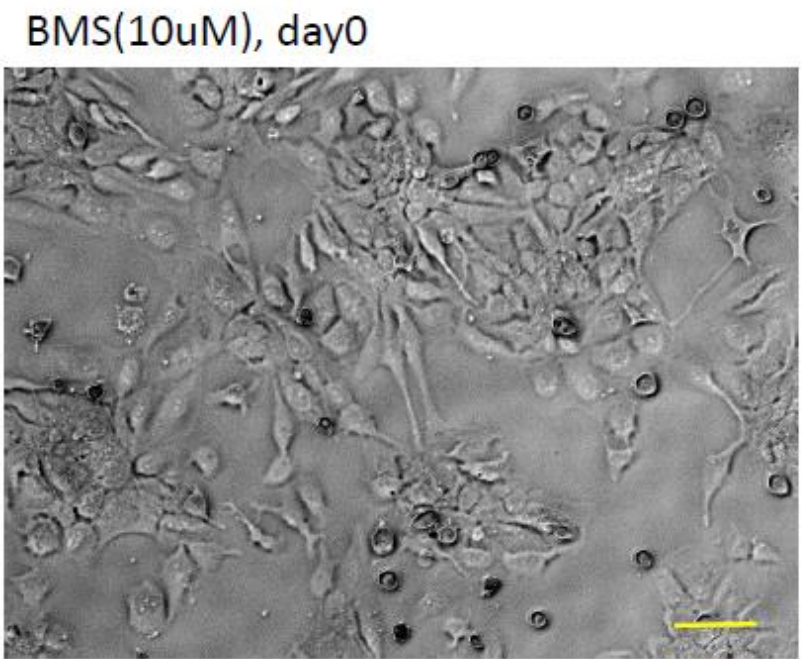

BMS(10uM), day1
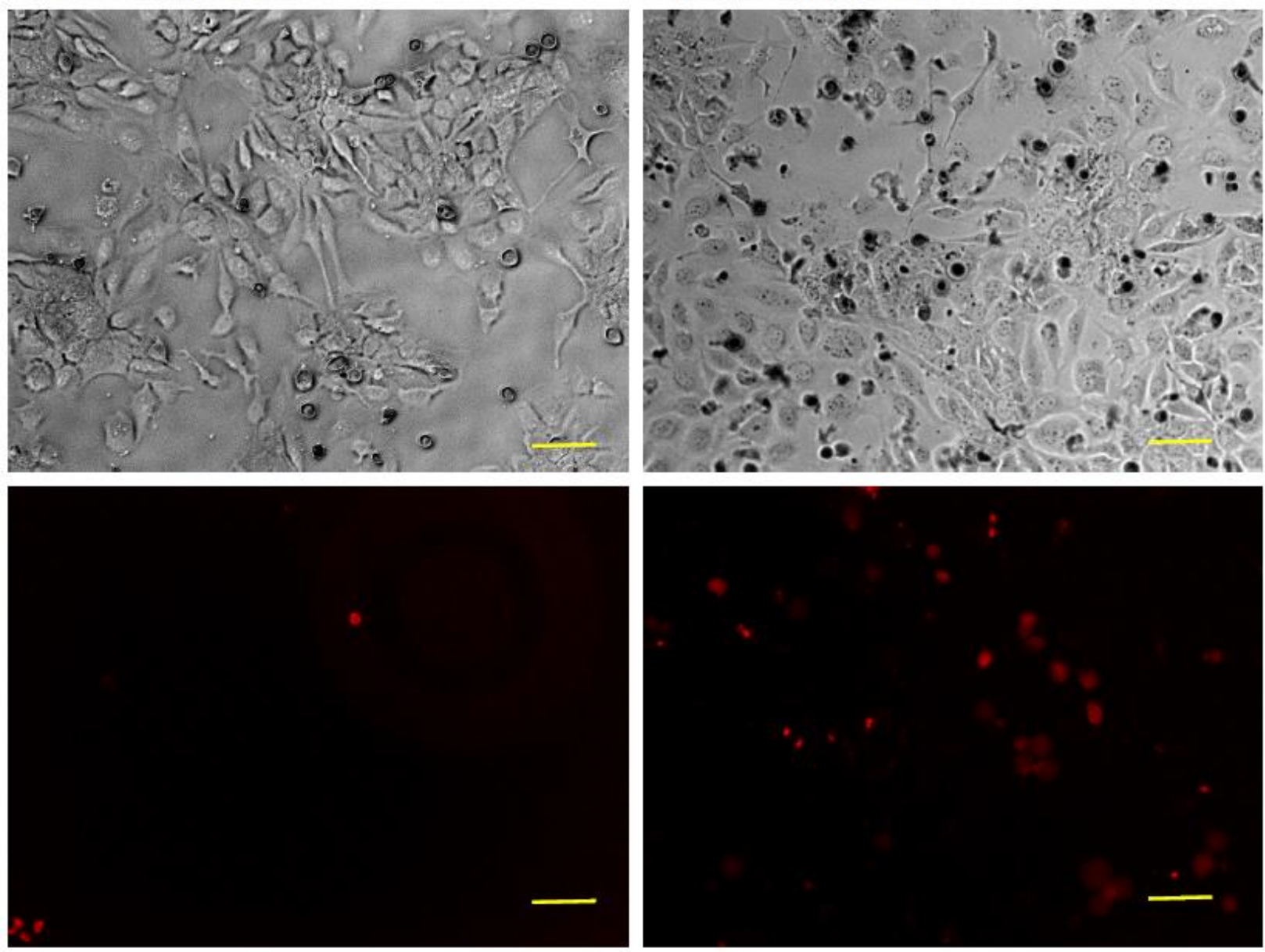

Supplemental Fig. 5 BMS-191011 on HCC1143. Upper panels show the effects of BMS-191011, lower panels show the dead cells labelled by EthD-1 dye (red). Left: control; Right: one day after $10 \square$ M BMS-191011 treatment. Scale bar: $20 \square \mathrm{m}$.

Supplemental Fig. 6 


\section{$0.5 \%$ DMSO}
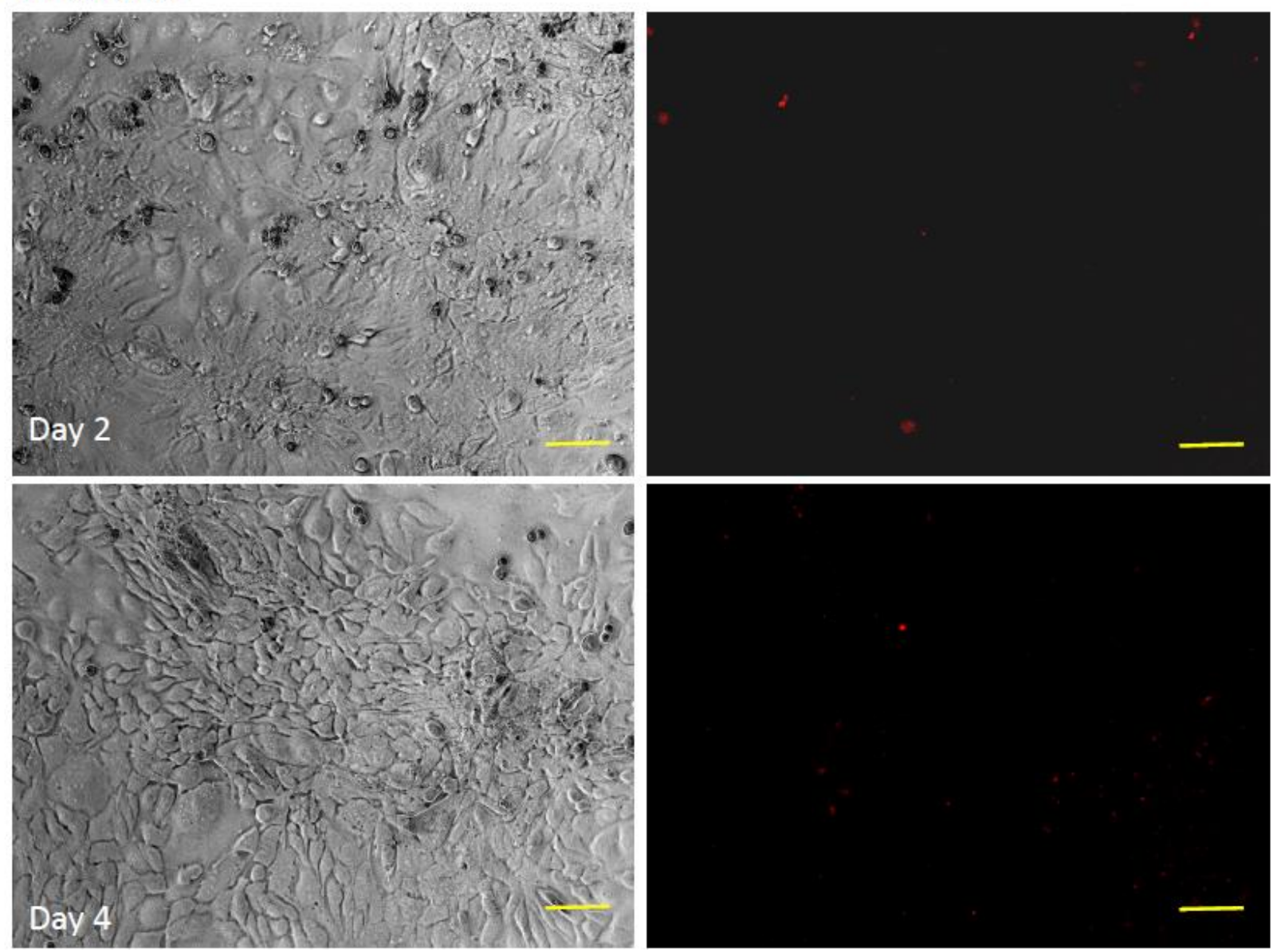

Supplemental Fig. 6 DMSO on HCC1143. DMSO (5 $\square$ 1, corresponding to the volume used for 50 $\square$ M BMS-191011) on HCC1143. Upper: after 48hr; Lower: after 96hr. Left: light image, Right: dead cells labelled by EthD-1 dye (red). Scale bar: $20 \square \mathrm{m}$.

\section{Supplemental Fig. 7}




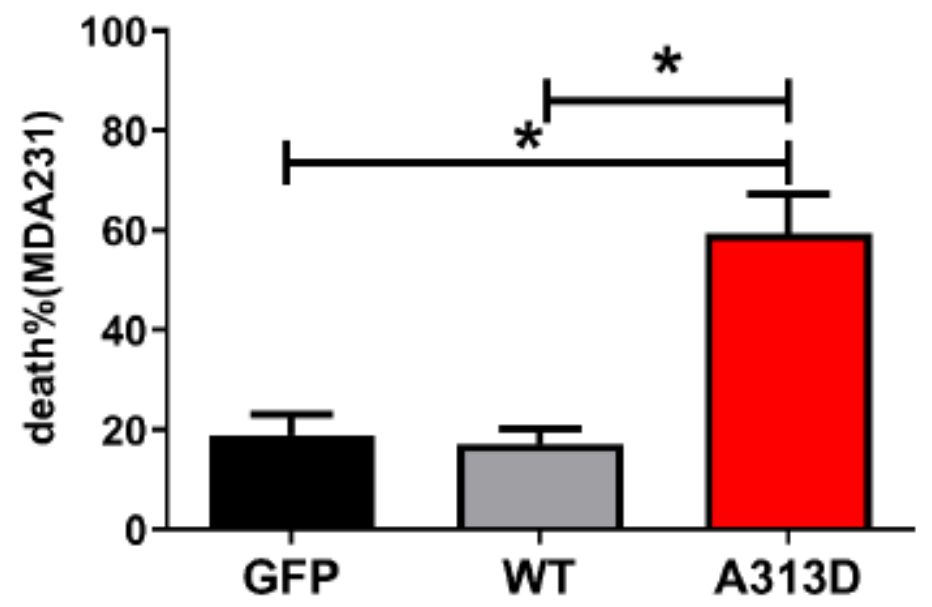

Supplemental Fig. 7 MDA-MB-231 cell death induced by a constitutively open BK channel mutant, A313D. WT: wild-type BK channel, A313D: mutant channel, GFP: GFP plasmid, *: p\&lt;0.05.

\section{Supplemental Fig. 8}
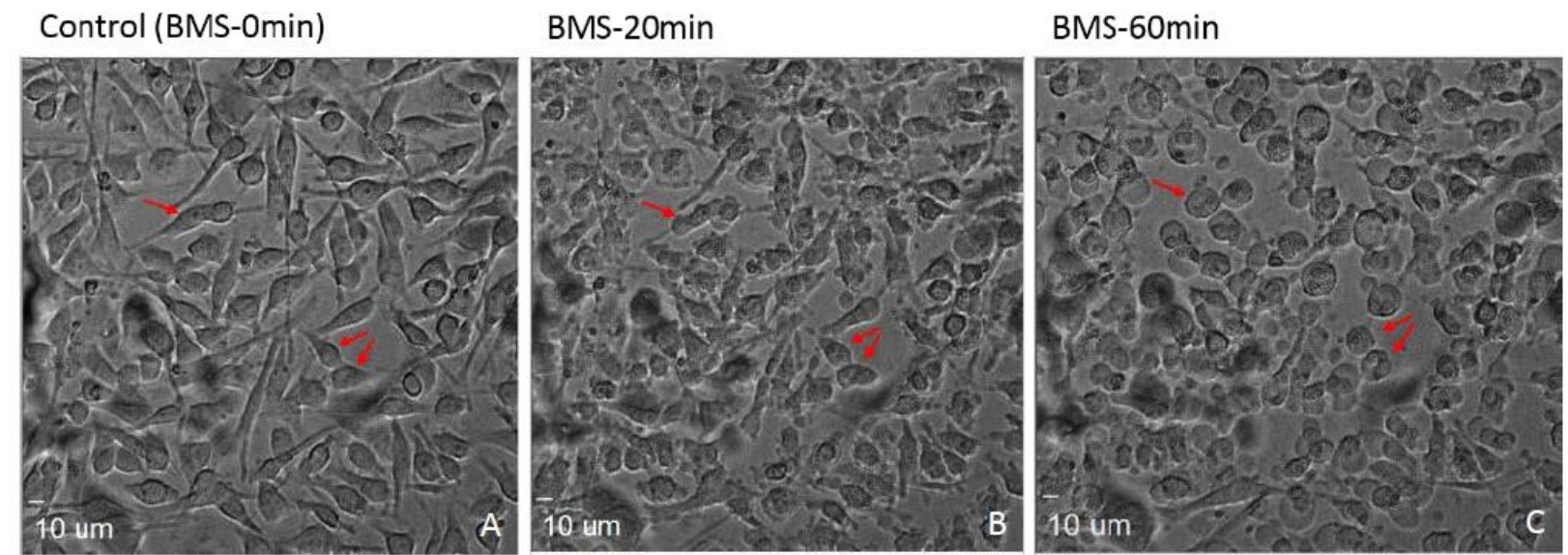

Supplemental Fig. 8 Three images corresponding to starting time point (control, $t=0)$ (A), 20min (B), and 60min (C) after BMS-191011 (1 $\square$ M) application are shown. Red arrows illustrate the representative cells undergoing morphological changes during the time course of BMS-191011.

\section{Supplemental Fig. 9}




\section{Con $\mathrm{T}=0$}

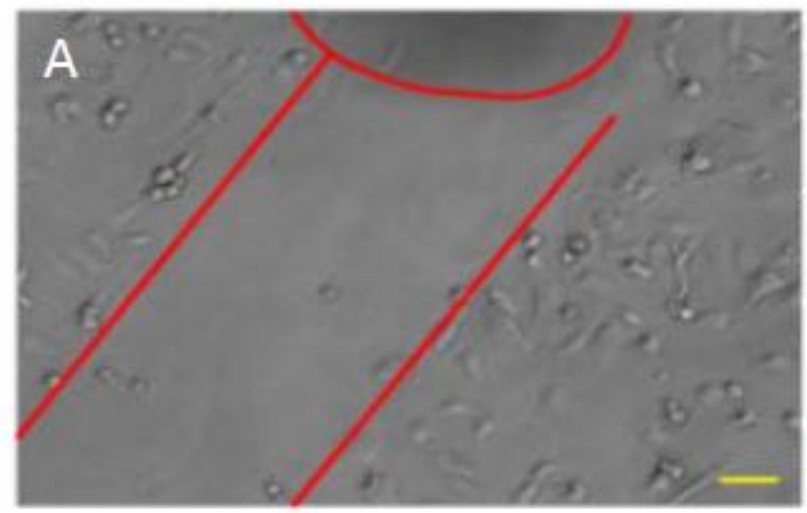

BMS-191011 100nM, T=0

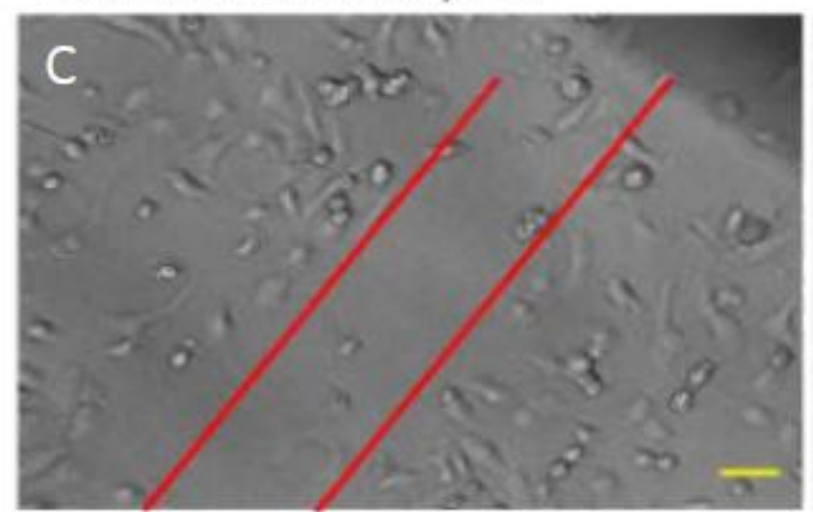

\section{Con $\mathrm{T}=4 \mathrm{hr}$}

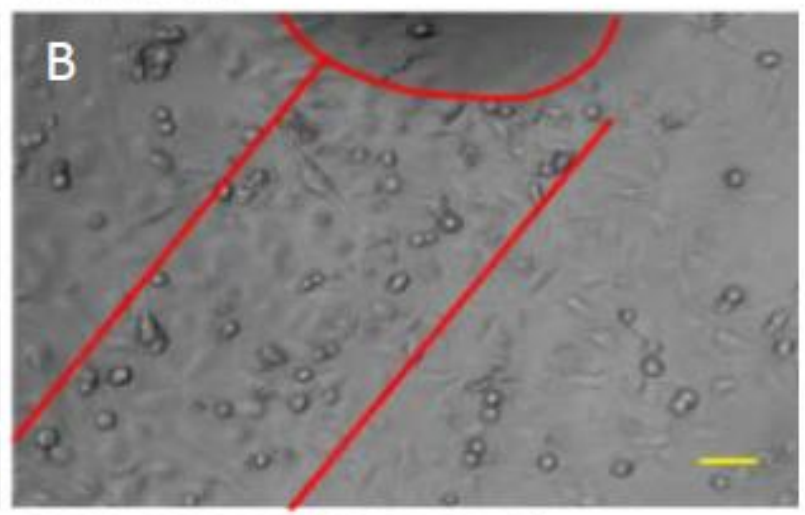

BMS-191011 100nM, T=4hr

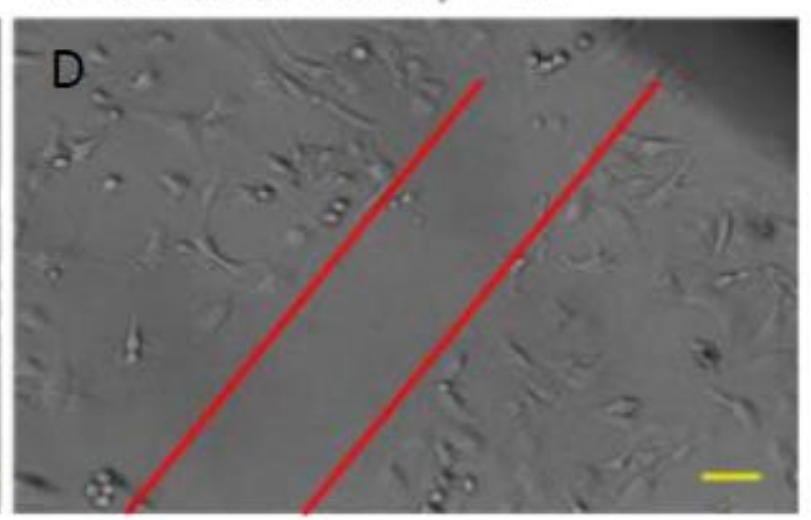

Supplemental Fig. 9 Prevention of MDA-MB-231 cells migration by BMS-

191011. In the absence of BK channel opener (Con) (A), cells migrated to fill the gap ("wound") after 4hrs (B). Low concentration (100nM) of BK-191011 (C) prevented the "heal" (cells filling the gap) after 4hrs (D). Scratch is defined by the space within two red lines. Curved red line indicates the marker (shadowed area) used to identify the location of the scratch. Scale bar: $20 \square \mathrm{m}$.

Supplemental Fig. 10 


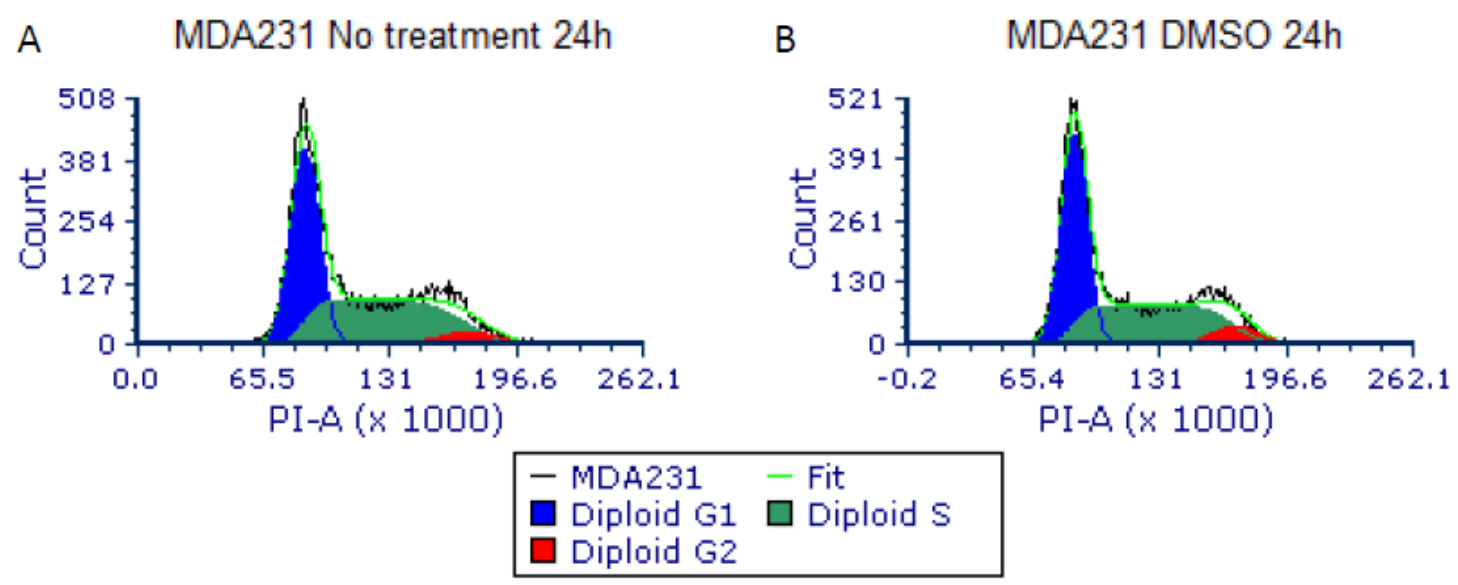

Supplemental Fig. 10 DMSO does not affect cell cycle in MDA-MB-231. a: no DMSO treatment, b: DMSO treatment after 24h. Blue: G1 phase; Green: S phase; Red: G2 phase. Count: amount of cells, PI-A: fluorescence intensity.

\section{Supplemental Fig. 11}

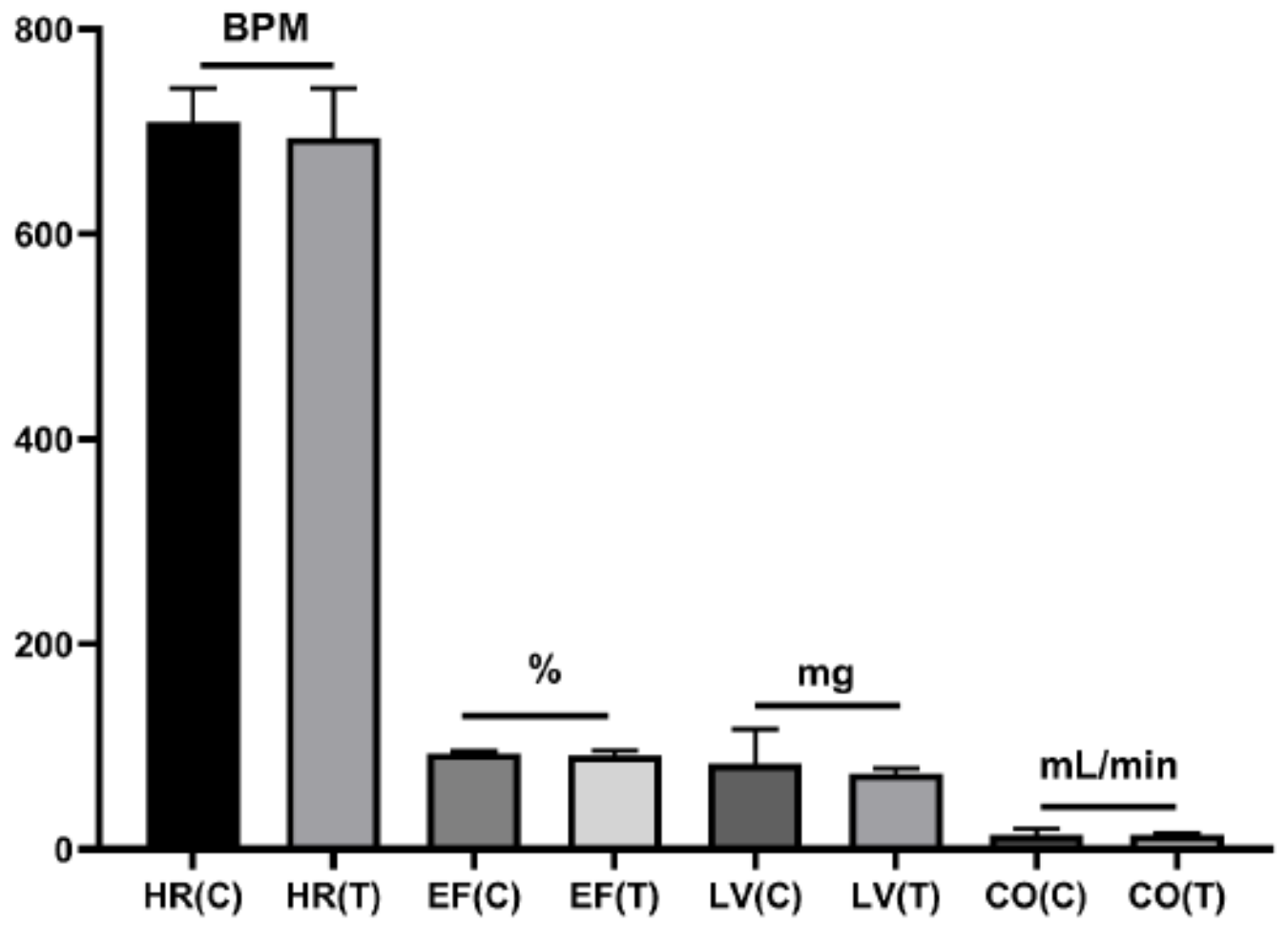

Supplemental Fig. 11 BK channel opener does not alter cardiac functions in NSG xenograft model. Results of three control and four treated mice were compared. C: control, T: BMS-191011 treated. HR: heart rate (beat per minute, BMP), EF: ejection fraction (\%), LV: 
corrected left ventricular mass $(\mathrm{mg}), \mathrm{CO}$ : cardiac output $(\mathrm{mL} / \mathrm{min})$. Unpaired t-Test was performed. For HR, $\mathrm{p}=0.6621$, $\mathrm{t}=0.4595$; For $\mathrm{EF}, \mathrm{p}=0.5281$, $\mathrm{t}=0.6695$; For $\mathrm{LV}, \mathrm{p}=0.5209$, $\mathrm{t}=0.6816$; For $\mathrm{CO}, \mathrm{p}=0.9443, \mathrm{t}=0.07285$.

\section{Supplemental Fig. 12}

A: MDA-MB-231/DsRed

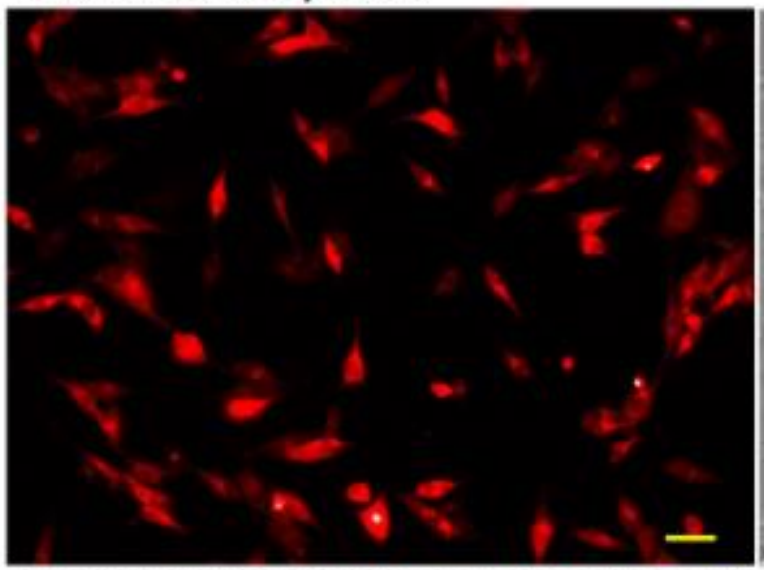

C: BMS-191011 20 $\mu \mathrm{M}$ (micro molar), Day 1

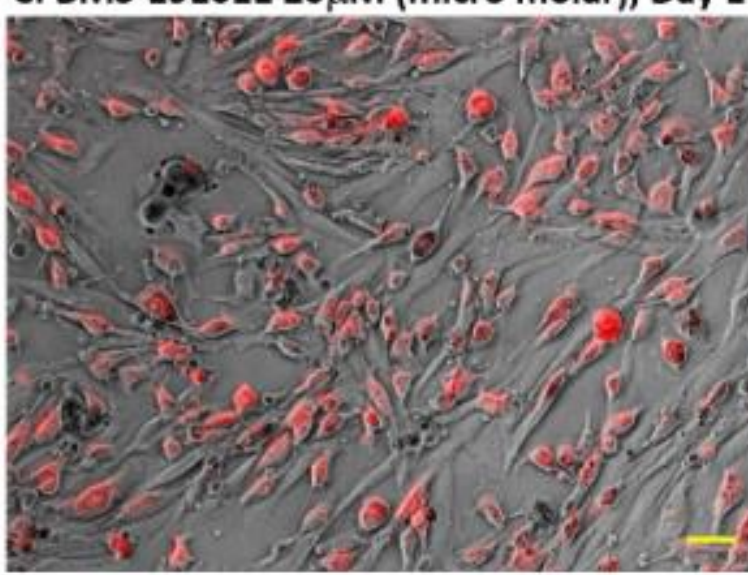

B: H9c2 cardiac myocytes

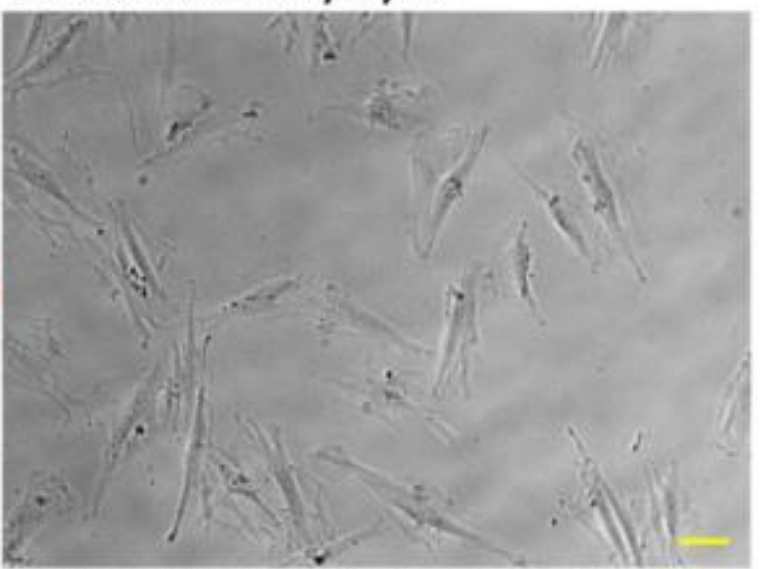

D: BMS-191011 20 $\mu \mathrm{M}$ (micro molar), Day 6

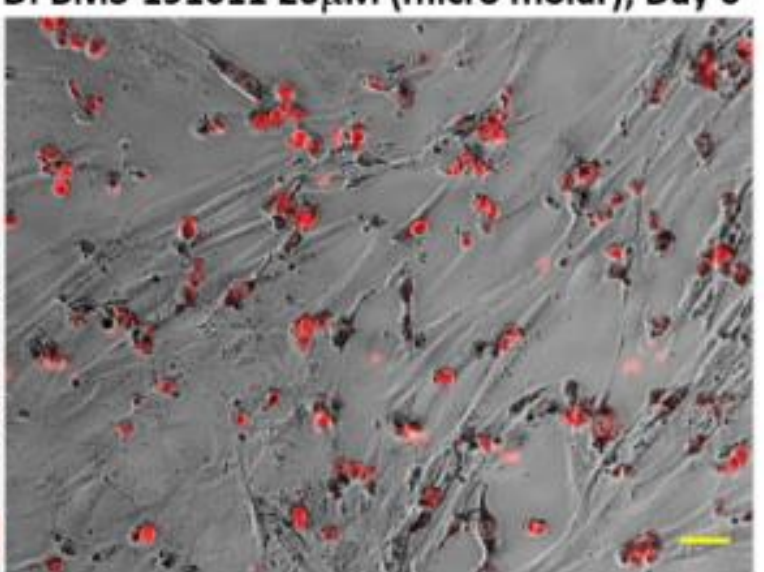

Supplemental Fig. 12 Supplemental Figure 13: BK channel opener induced cell death in MDAMB-231, but not in cardiac myocytes. A: MDA-MB-231 stable cell line with DsRed inserted. B: H9c2 cardiac myocytes. C: co-culture of MDA-MB-231/DsRed cells (red) with H9c2 cardiac myocytes (gray) after one-day treatment of BMS-191011 (20 $\square \mathrm{M})$. D: co-culture of MDA-MB-231/DsRed cells (red) with H9c2 cardiac myocytes (gray) after six-day treatment of BMS-191011 (20 $\square \mathrm{M})$. Scale bar: $30 \square \mathrm{m}$. 
Triple Negative Breast Cancer: Exponential Tumor Grade Increase with Age of Diagnosis

Table 1

\begin{tabular}{|c|c|c|c|c|c|c|}
\hline & TN & & Non- & $\mathrm{BC}$ & To & \\
\hline Characteristic & $\mathrm{N}$ & $\%$ & $\mathrm{~N}$ & $\%$ & $\mathrm{~N}$ & $\%$ \\
\hline \multicolumn{7}{|l|}{ Age group } \\
\hline$\leq 40$ & 81 & 7.0 & 318 & 4.0 & 399 & 4.4 \\
\hline $41-50$ & 205 & 17.6 & 1,055 & 13.3 & 1,260 & 13.9 \\
\hline $51-60$ & 290 & 24.9 & 1,832 & 23.1 & 2,122 & 23.3 \\
\hline $61-70$ & 317 & 27.2 & 2,326 & 29.3 & 2,643 & 29.0 \\
\hline 271 & 273 & 23.4 & 2,403 & 30.3 & 2,676 & 29.4 \\
\hline Missing & 0 & & 0 & & & \\
\hline \multicolumn{7}{|l|}{ Race } \\
\hline White & 1,098 & 94.2 & 7665 & 96.7 & 8,763 & 96.3 \\
\hline Other & 68 & 5.8 & 264 & 3.3 & 332 & 3.7 \\
\hline \multicolumn{7}{|l|}{$\begin{array}{l}\text { Year of } \\
\text { diagnosis }\end{array}$} \\
\hline 2010 & 167 & 14.3 & 979 & 12.3 & 1,146 & 12.6 \\
\hline 2011 & 171 & 14.7 & 1,052 & 13.3 & 1,223 & 13.4 \\
\hline 2012 & 161 & 13.8 & 1,138 & 14.3 & 1,299 & 14.3 \\
\hline 2013 & 172 & 14.8 & 1,172 & 14.8 & 1,344 & 14.8 \\
\hline 2014 & 151 & 13.0 & 1,166 & 14.7 & 1,317 & 14.5 \\
\hline 2015 & 185 & 15.9 & 1,197 & 15.1 & 1,382 & 15.2 \\
\hline 2016 & 159 & 13.6 & 1,230 & 15.5 & 1,389 & 15.3 \\
\hline \multicolumn{7}{|l|}{$\begin{array}{l}\text { Stage at } \\
\text { diagnosis }\end{array}$} \\
\hline Local & 734 & 63.4 & 5,258 & 66.7 & 5,992 & 66.2 \\
\hline Regional direct & 26 & 2.3 & 151 & 1.9 & 177 & 2.0 \\
\hline $\begin{array}{l}\text { Regional } \\
\text { lymph }\end{array}$ & 255 & 22.0 & 1817 & 23.0 & 2,072 & 22.9 \\
\hline $\begin{array}{r}\text { Direct and } \\
\text { lymph }\end{array}$ & 49 & 4.2 & 214 & 2.7 & 263 & 2.9 \\
\hline Distant & 94 & 8.1 & 448 & 5.7 & 542 & 6.0 \\
\hline
\end{tabular}




\section{Table 1 Continued}

\begin{tabular}{|c|c|c|c|c|c|c|}
\hline Unknown & 8 & & 46 & & 54 & \\
\hline \multicolumn{7}{|l|}{ Metastasis } \\
\hline Yes & 73 & 6.9 & 389 & 5.4 & 7,867 & 94.5 \\
\hline No & 993 & 93.1 & 6874 & 94.6 & 462 & 5.6 \\
\hline Missing & 100 & & 671 & & 771 & \\
\hline \multicolumn{7}{|l|}{$\begin{array}{l}\text { Node } \\
\text { involvement }\end{array}$} \\
\hline Yes & 283 & 29.8 & 1,985 & 29.6 & 2,268 & 29.6 \\
\hline No & 668 & 70.2 & 4,730 & 70.4 & 5,398 & 70.4 \\
\hline Missing & 215 & & 1,219 & & 1,434 & \\
\hline \multicolumn{7}{|l|}{ Insitu } \\
\hline Yes & 3 & 0.3 & 15 & 0.2 & 18 & 0.2 \\
\hline No & 1,093 & 99.7 & 7,599 & 99.8 & 8,692 & 99.8 \\
\hline Missing & 70 & & 320 & & 390 & \\
\hline \multicolumn{7}{|l|}{ Tumor grade } \\
\hline Low & 60 & 5.6 & 2,164 & 29.2 & 2,224 & 26.3 \\
\hline Moderate & 226 & 21.3 & 3,571 & 48.2 & 3,797 & 44.8 \\
\hline High & 777 & 73.1 & 1,670 & 22.6 & 2,447 & 28.9 \\
\hline Missing & 103 & & 529 & & 632 & \\
\hline \multicolumn{7}{|l|}{$\begin{array}{l}\text { Multigene test } \\
\text { performed }\end{array}$} \\
\hline Yes & 49 & 5.8 & 1,759 & 28.8 & 1,808 & 26.0 \\
\hline No & 802 & 94.2 & 4,346 & 71.2 & 5,148 & 74.0 \\
\hline Missing & 315 & & 1,829 & & & \\
\hline \multicolumn{7}{|l|}{ Pagets } \\
\hline Yes & 6 & 0.7 & 62 & 1.0 & 68 & 1.0 \\
\hline No & 900 & 99.3 & 5,960 & 99.0 & 6,860 & 99.0 \\
\hline Missing & 260 & & 1,912 & & 2,172 & \\
\hline
\end{tabular}




\section{Table 2}

\begin{tabular}{|c|c|c|c|c|c|c|}
\hline \multirow[b]{2}{*}{ Characteristic } & \multirow[t]{2}{*}{ Total N } & \multirow{2}{*}{$\begin{array}{c}\% \text { of } \\
\text { population } \\
\text { with TNBC }\end{array}$} & \multicolumn{2}{|c|}{ Model 1} & \multicolumn{2}{|r|}{ Model 2} \\
\hline & & & $\mathrm{OR}$ & $95 \% \mathrm{Cl}$ & OR & $95 \% \mathrm{Cl}$ \\
\hline \multicolumn{7}{|l|}{ Age group } \\
\hline$\leq 40$ & 399 & 20.3 & 2.24 & $1.70,2.95$ & 2.19 & $1.67,2.88$ \\
\hline $41-50$ & 1,260 & 16.3 & 1.71 & $1.41,2.08$ & 1.68 & $1.38,2.05$ \\
\hline $51-60$ & 2,122 & 13.7 & 1.39 & $1.17,1.66$ & 1.38 & $1.15,1.64$ \\
\hline $61-70$ & 2,643 & 12.0 & 1.20 & $1.01,1.42$ & 1.20 & $1.01,1.42$ \\
\hline 271 & 2,676 & 10.2 & 1.00 & Referent & 1.00 & Referent \\
\hline \multicolumn{7}{|l|}{ Race } \\
\hline White & 8,763 & 12.5 & 1.00 & Referent & 1.00 & Referent \\
\hline Other & 332 & 20.5 & 1.80 & $1.38,2.37$ & 1.71 & $1.30,2.26$ \\
\hline \multicolumn{7}{|l|}{ Node Involvement } \\
\hline Yes & 2,268 & 12.5 & 1.01 & $0.87,1.17$ & 0.95 & $0.81,1.10$ \\
\hline No & 5,398 & 12.4 & 1.00 & Referent & 1.00 & Referent \\
\hline \multicolumn{7}{|l|}{ Insitu } \\
\hline Yes & 18 & 16.7 & 1.39 & $0.40,4.81$ & 1.58 & $0.46,5.49$ \\
\hline No & 8,692 & 12.6 & 1.00 & Referent & 1.00 & Referent \\
\hline \multicolumn{7}{|l|}{ Tumor grade } \\
\hline Low & 2,224 & 2.7 & 1.00 & Referent & 1.00 & Referent \\
\hline Moderate & 3,797 & 6.0 & 2.28 & $1.71,3.05$ & 2.27 & $1.70,3.03$ \\
\hline High & 2,447 & 31.8 & 16.78 & $12.81,21.98$ & 16.24 & $12.38,21.30$ \\
\hline \multicolumn{7}{|l|}{ Metastasis } \\
\hline Yes & 462 & 15.8 & 1.30 & $1.00,1.68$ & 1.29 & $0.99,1.67$ \\
\hline No & 7,867 & 12.6 & 1.00 & Referent & 1.00 & Referent \\
\hline \multicolumn{7}{|l|}{ Multigene } \\
\hline Yes & 1,808 & 2.7 & 0.15 & \begin{tabular}{|l|}
$0.11,0.20$ \\
\end{tabular} & 0.14 & \begin{tabular}{|l|l|}
$0.11,0.19$ \\
\end{tabular} \\
\hline No & 5,148 & 15.6 & 1.00 & Referent & 1.00 & Referent \\
\hline \multicolumn{7}{|l|}{ Paget } \\
\hline Yes & 68 & 8.8 & 0.64 & $0.28,1.49$ & 0.66 & $0.28,1.53$ \\
\hline No & 6,860 & 13.1 & 1.00 & Referent & 1.00 & Referent \\
\hline
\end{tabular}




\section{Table 3}

\begin{tabular}{|c|c|c|}
\hline \multirow{3}{*}{$\begin{array}{l}\text { Grade by Age group } \\
\leq 40\end{array}$} & \multicolumn{2}{|c|}{ Model 1} \\
\hline & OR & $95 \% \mathrm{Cl}$ \\
\hline & & \\
\hline Low & 1.00 & Referent \\
\hline Moderate & 1.16 & $0.36,3.73$ \\
\hline \multirow[t]{2}{*}{ High } & 5.41 & $1.85,15.80$ \\
\hline & \multicolumn{2}{|c|}{$41-50$} \\
\hline Low & 1.00 & Referent \\
\hline Moderate & 1.12 & $0.55,2.27$ \\
\hline \multirow[t]{2}{*}{ High } & 10.48 & $5.67,19.39$ \\
\hline & \multicolumn{2}{|c|}{$51-60$} \\
\hline Low & 1.00 & Referent \\
\hline Moderate & 1.89 & $1.03,3.46$ \\
\hline $\begin{array}{r}\text { Moderate } \\
\text { High }\end{array}$ & 14.61 & $8.37,25.52$ \\
\hline \multicolumn{3}{|l|}{$61-70$} \\
\hline Low & 1.00 & Referent \\
\hline Moderate & 2.54 & $1.46,4.42$ \\
\hline High & 20.17 & $11.96,34.02$ \\
\hline \multicolumn{3}{|l|}{271} \\
\hline Low & 1.00 & Referent \\
\hline Moderate & 3.64 & $2.04,6.50$ \\
\hline High & 21.73 & $12.42,38.03$ \\
\hline \multicolumn{3}{|c|}{$\begin{array}{l}\text { a: Model was adjusted for year and race. The } \\
\text { outcome was whether the patient was diagnosed } \\
\text { for triple negative breast cancer vs. regular breast } \\
\text { cancer. The primary independent variable was } \\
\text { tumor grade at diagnosis, which is stratified by } \\
\text { age group }\end{array}$} \\
\hline
\end{tabular}




\section{Table 4}

\begin{tabular}{|c|c|c|}
\hline & \multicolumn{2}{|c|}{ Model 1} \\
\hline $\begin{array}{l}\text { Multigene test by age } \\
\text { group }\end{array}$ & OR & $95 \% \mathrm{Cl}$ \\
\hline \multicolumn{3}{|l|}{$\leq 40$} \\
\hline Yes & 0.52 & $0.24,1.13$ \\
\hline No & 1.00 & Referent \\
\hline \multicolumn{3}{|l|}{$41-50$} \\
\hline Yes & 0.25 & $0.15,0.43$ \\
\hline No & 1.00 & Referent \\
\hline \multicolumn{3}{|l|}{$51-60$} \\
\hline Yes & 0.05 & $0.02,0.11$ \\
\hline No & 1.00 & Referent \\
\hline \multicolumn{3}{|l|}{$61-70$} \\
\hline Yes & 0.11 & $0.07,0.19$ \\
\hline No & 1.00 & Referent \\
\hline \multicolumn{3}{|l|}{271} \\
\hline Yes & 0.12 & $0.04,0.32$ \\
\hline No & 1.00 & Referent \\
\hline \multicolumn{3}{|c|}{$\begin{array}{l}\text { a: Model was adjusted for year and race. The outcome } \\
\text { was whether the patient was diagnosed for triple } \\
\text { negative breast cancer vs. regular breast cancer. The } \\
\text { primary independent variable was whether the patient } \\
\text { received multigene therapy, which is stratified by age } \\
\text { group }\end{array}$} \\
\hline
\end{tabular}




\section{CHAPTER NINE}

\section{Conclusion}

This composition of three projects reflects the perspective of clinical translational science. The first work reviews the use of electrophysiology in the microscopic and macroscopic world, demonstrates a novel means of connecting them with this tool, and discusses opportunities for future research on a broad scale. The second work tackles application of electrophysiology to identify a novel targeted treatment for an aggressive breast cancer disease, triple negative breast cancer. Finally, the electrophysiology discovery guides the hypothesis for an epidemiology study to determine the use of molecular signature testing for triple negative breast cancer in the West Virginia population to prepare the way for future research identifying biomarkers and targets for this disease.

Electrophysiology in Clinical Translational Science creates novel opportunity for translating basic science to the clinic by virtue of a tool that communicates data across the chasm between the microscopic scale of bench science and the macroscopic scale of clinical application. This tool creates novel application of electrophysiology for disease evaluation across measures of scale. It provides functional evaluation of genetic data regarding over-expressed and underexpressed ion channels to guide discovery for disease treatment. Finally, it advances epidemiologic discovery by generation of novel insight for researching disease in the population. Utilizing electrophysiology to bridge the gap between microscopic basic science discovery and macroscopic clinical application is a difficult challenge. This project seeks to recommend growth in the use of electrophysiology beyond the boundaries of this work, demonstrate the novel use of it in development of targeted treatment for a disease, and exhibit its novel provision to epidemiologic guidance of disease research in the population. 
In addition, the ability of electrophysiology in ion channel evaluation to resolve a significant contradiction in the literature of BK ion channels demonstrates its formidable ability to give functional quantitative answers to the ambiguity of genetic expression. Prior to this research controversial results of the BK channel in breast cancer proliferation and treatment were blatant. Khaitan et al in his 2009 work demonstrated that metastatic brain cancer from breast overexpressed BK channels more than metastatic cancer from the breast to other organs. From this he concluded that the migration and invasion was due to the increase of BK channel and that blocking the channel would be an effective way to impact this process. ${ }^{48}$ However, what was overlooked is the function of the channel. Our research has demonstrated that the BK channel is not open (active) at physiologic voltage therefore inhibition of a closed channel is unlikely to produce the impact anticipated.

Indeed, research by additional scientist have demonstrated that inhibition did not have the anticipated impact. Schickling's discussion in Oncogene Report regarding their equivocal results caused him to note that BK channels may be pro or against growth. ${ }^{49}$ The significant contribution of this dissertation to facilitate a final answer to this controversy not only gives perspective to the cause of the contradictions (ie...not evaluating channel function) but also provides solid, reproducible techniques for future ion channel research in diseases with over and under expressed ion channels.

Finally, the in vitro altered homeostasis in ion channel targeting of a single cell of human triple negative breast cancer and the in vivo impact of its application in mice can be discussed with clinicians using the language of electrophysiology regarding polarization, depolarization, and hyperpolarization, a language they already speak with EKG analysis in their daily routines. It is this hyperpolarization which is the mechanism of cell death when the overexpressed BK 
channels are forced open and potassium efflux leads to apoptosis. The evidence for potassium efflux as mechanism for apoptosis is well demonstrated. ${ }^{50}$ Our research utilizing electrophysiology demonstrates hyperpolarization to confirm potassium efflux via the BK channel as mechanism of apoptosis in triple negative breast cancer. Therefore, this genetic variation of overexpression can be exploited to target the disease. Electrophysiology gives functional answers to the decades of accumulated genetic research. Patch clamp, as an electrophysiology tool speaks in the physics language of electrical current which translates from basic science to the clinic.

The language barrier electrophysiology bridges between basic science and clinical medicine offers a significant opportunity to utilize a tool that will give functional data to our growing collection of genetic data that can be translated to clinical application. The initial manuscript in this dissertation work discussed interconnected evaluations of multiple ions and ion channels that is still very difficult to do and will require extensive modeling to fully develop. However, the second manuscript in this dissertation demonstrates a beginning step in that direction by utilizing a transmembrane channel that operates by interaction with two ions; the ion transported across the membrane -potassium - and the ion that impacts its gating, calcium. The evidence of the BK channel to selectively kill triple negative breast cancer in-vitro and halt tumor growth in-vivo is a novel discovery for targeted treatment of triple negative breast cancer with BK channel openers that addresses the contradiction of previous research which only considered its overexpression not its electrophysiologic activity at physiologic states. This new evidence demonstrates BK channel opportunity as a targeted treatment and confirms the role of calcium in proliferation rather than the BK channel which is simply impacted by the increased calcium. Finally, the third manuscript provides evidence for decreased utilization of tests, such as the multigene signature 
test, which can identify molecular biology variants like overexpression, and response to treatment. Together these create an opportunity for explosive translational growth as we wield these discoveries into additional research and change. 


\section{CHAPTER TEN}

\section{References}

1. Morris, Z. S., Wooding, S. \& Grant, J. The answer is 17 years, what is the question: understanding time lags in translational research. J. R. Soc. Med. 104, 510-520 (2011).

2. Marino, A. A. et al. Association between cell membrane potential and breast cancer. Tumour Biol. J. Int. Soc. Oncodevelopmental Biol. Med. 15, 82-89 (1994).

3. Berzingi, S., Newman, M. \& Yu, H.-G. Altering bioelectricity on inhibition of human breast cancer cells. Cancer Cell Int. 16, 72 (2016).

4. Yang, M. \& Brackenbury, W. J. Membrane potential and cancer progression. Front. Physiol. 4, 185 (2013).

5. Alexander, L. K., Lopes, B., Ricchetti-Masterson, K. \& Yeatts, K. B. Confounding Bias, Part II and Effect Measure Modification. 7.

6. Lorca, R. A. et al. N-terminal Isoforms of the Large-conductance Ca2+-activated K+ Channel Are Differentially Modulated by the Auxiliary ß1-Subunit. J. Biol. Chem. 289, 10095-10103 (2014).

7. Cheng, Y. Y. et al. KCa1.1, a calcium-activated potassium channel subunit alpha 1 , is targeted by miR17-5p and modulates cell migration in malignant pleural mesothelioma. Mol. Cancer 15, 44 (2016).

8. Reference, G. H. KCNMA1 gene. Genetics Home Reference https://ghr.nIm.nih.gov/gene/KCNMA1.

9. Latorre, R., Morera, F. J. \& Zaelzer, C. SYMPOSIUM REVIEW: Allosteric interactions and the modular nature of the voltage- and Ca2+-activated (BK) channel. J. Physiol. 588, 3141-3148 (2010).

10. Kshatri, A. S., Gonzalez-Hernandez, A. J. \& Giraldez, T. Functional validation of Ca2+-binding residues from the crystal structure of the BK ion channel. Biochim. Biophys. Acta Biomembr. 1860, 943-952 (2018).

11. Sun, X.-P., Schlichter, L. C. \& Stanley, E. F. Single-channel properties of BK-type calcium-activated potassium channels at a cholinergic presynaptic nerve terminal. J. Physiol. 518, 639-651 (1999). 
12. Zeng, X., Xia, X.-M. \& Lingle, C. J. Species-specific Differences among KCNMB3 BK beta3 auxiliary subunits: some beta3 N-terminal variants may be primate-specific subunits. J. Gen. Physiol. 132, 115129 (2008).

13. Tang, Q.-Y., Zhang, Z., Xia, X.-M. \& Lingle, C. J. Block of mouse Slo1 and Slo3 K+ channels by CTX, IbTX, TEA, 4-AP and quinidine. Channels 4, 22-41 (2010).

14. Lee, U. S. \& Cui, J. BK channel activation: structural and functional insights. Trends Neurosci. 33, 415-423 (2010).

15. Chen, X., Yan, J. \& Aldrich, R. W. BK channel opening involves side-chain reorientation of multiple deep-pore residues. Proc. Natl. Acad. Sci. U. S. A. 111, E79-E88 (2014).

16. Li, Q. \& Yan, J. Modulation of BK Channel Function by Auxiliary Beta and Gamma Subunits. Int. Rev. Neurobiol. 128, 51-90 (2016).

17. Knaus, H.-G. et al. Characterization of Tissue-expressed $\alpha$ Subunits of the High Conductance Ca2+-activated K+ Channel. J. Biol. Chem. 270, 22434-22439 (1995).

18. Mori, A., Suzuki, S., Sakamoto, K., Nakahara, T. \& Ishii, K. BMS-191011, an Opener of LargeConductance Ca2+-Activated Potassium Channels, Dilates Rat Retinal Arterioles in Vivo. Biol. Pharm. Bull. 34, 150-152 (2011).

19. Wright, S. H. Generation of resting membrane potential. Adv. Physiol. Educ. 28, 139-142 (2004).

20. Vetri, F., Choudhury, M. S. R., Pelligrino, D. A. \& Sundivakkam, P. BK ${ }_{\mathrm{Ca}}$ channels as physiological regulators: a focused review. Journal of Receptor, Ligand and Channel Research https://www.dovepress.com/bkca-channels-as-physiological-regulators-a-focused-review-peerreviewed-fulltext-article-JRLCR (2014) doi:10.2147/JRLCR.S36065.

21. Lai, M. H. et al. BK channels regulate sinoatrial node firing rate and cardiac pacing in vivo. Am. J. Physiol.-Heart Circ. Physiol. 307, H1327-H1338 (2014). 
22. Chavez, K. J., Garimella, S. V. \& Lipkowitz, S. Triple Negative Breast Cancer Cell Lines: One Tool in the Search for Better Treatment of Triple Negative Breast Cancer. Breast Dis. 32, 35-48 (2010).

23. Neve, R. M. et al. A collection of breast cancer cell lines for the study of functionally distinct cancer subtypes. Cancer Cell 10, 515-527 (2006).

24. Kao, J. et al. Molecular Profiling of Breast Cancer Cell Lines Defines Relevant Tumor Models and Provides a Resource for Cancer Gene Discovery. PLOS ONE 4, (2009).

25. Prat, A. \& Perou, C. Deconstructing the molecular portraits of breast cancer. Mol. Oncol. 5, 5-23 (2010).

26. Dai, X., Cheng, H., Bai, Z. \& Li, J. Breast Cancer Cell Line Classification and Its Relevance with Breast Tumor Subtyping. J. Cancer 8, 3131-3141 (2017).

27. Sporikova, Z., Koudelakova, V., Trojanec, R. \& Hajduch, M. Genetic Markers in Triple-Negative Breast Cancer. Clin. Breast Cancer (2018) doi:10.1016/j.clbc.2018.07.023.

28. Aysola, K. et al. Triple Negative Breast Cancer - An Overview. Hered. Genet. Curr. Res. 2013, (2013).

29. Lehmann, B. D. et al. Refinement of Triple-Negative Breast Cancer Molecular Subtypes: Implications for Neoadjuvant Chemotherapy Selection. PloS One 11, e0157368 (2016).

30. He, Y., Jiang, Z., Chen, C. \& Wang, X. Classification of triple-negative breast cancers based on Immunogenomic profiling. J. Exp. Clin. Cancer Res. 37, 327 (2018).

31. Smith, S. E. et al. Molecular characterization of breast cancer cell lines through multiple omic approaches. Breast Cancer Res. 19, 65 (2017).

32. Nie, L. et al. CDK2-mediated site-specific phosphorylation of EZH2 drives and maintains triplenegative breast cancer. Nat. Commun. 10, 1-15 (2019).

33. Lanning, N. J. et al. Metabolic profiling of triple-negative breast cancer cells reveals metabolic vulnerabilities. Cancer Metab. 5, (2017). 
34. Wu, X. et al. Global phosphotyrosine survey in triple-negative breast cancer reveals activation of multiple tyrosine kinase signaling pathways. Oncotarget 6, 29143-29160 (2015).

35. Syed, S., Schober, J., Blanco, A. \& Zustiak, S. P. Morphological adaptations in breast cancer cells as a function of prolonged passaging on compliant substrates. PLOS ONE 12, (2017).

36. Domura, R., Sasaki, R., Ishikawa, Y. \& Okamoto, M. Cellular Morphology-Mediated Proliferation and Drug Sensitivity of Breast Cancer Cells. J. Funct. Biomater. 8, (2017).

37. Hodgkin, A. L. \& Huxley, A. F. Currents carried by sodium and potassium ions through the membrane of the giant axon of Loligo. J. Physiol. 116, 449-472.

38. Breast Cancer.Org. Breast Cancer Metastasis to Brain: Symptoms and Diagnosis.

Breastcancer.org https://www.breastcancer.org/symptoms/types/recur_metast/metastic/brain (2019).

39. Niwińska, A., Olszewski, W., Murawska, M. \& Pogoda, K. Triple-negative breast cancer with brain metastases: a comparison between basal-like and non-basal-like biological subtypes. J. Neurooncol. 105, 547-553 (2011).

40. American Cancer Society. Triple-negative Breast Cancer | Details, Diagnosis, and Signs. https://www.cancer.org/cancer/breast-cancer/understanding-a-breast-cancer-diagnosis/types-ofbreast-cancer/triple-negative.html.

41. Radosa, J. C. et al. Evaluation of Local and Distant Recurrence Patterns in Patients with TripleNegative Breast Cancer According to Age. Ann. Surg. Oncol. 24, 698-704 (2017).

42. Pawar, A. \& Prabhu, P. Nanosoldiers: A promising strategy to combat triple negative breast cancer. Biomed. Pharmacother. 110, 319-341 (2019).

43. Lehmann, B. D. et al. Identification of human triple-negative breast cancer subtypes and preclinical models for selection of targeted therapies. J. Clin. Invest. 121, 2750-2767 (2011). 
44. Wahba, H. A. \& El-Hadaad, H. A. Current approaches in treatment of triple-negative breast cancer. Cancer Biol. Med. 12, 106-116 (2015).

45. Kim, K. et al. Breast Conservation Therapy Versus Mastectomy in Patients with T1-2N1 TripleNegative Breast Cancer: Pooled Analysis of KROG 14-18 and 14-23. Cancer Res. Treat. Off. J. Korean Cancer Assoc. 50, 1316-1323 (2018).

46. Yao, Y., Chu, Y., Xu, B., Hu, Q. \& Song, Q. Radiotherapy after surgery has significant survival benefits for patients with triple-negative breast cancer. Cancer Med. 8, 554-563 (2019).

47. SEER. National Cancer Institute. SEER Registries - About SEER. SEER https://seer.cancer.gov/registries/index.html.

48. Khaitan, D. et al. Role of KCNMA1gene in breast cancer invasion and metastasis to brain. BMC Cancer 9, 258 (2009).

49. Schickling, B. M. et al. BKCa channel inhibitor modulates the tumorigenic ability of hormoneindependent breast cancer cells via the Wnt pathway. Oncol. Rep. 33, 533-538 (2015).

50. Bortner, C. D., Hughes, F. M. \& Cidlowski, J. A. A Primary Role for $\mathrm{K}^{+}$and $\mathrm{Na}^{+}$Efflux in the Activation of Apoptosis. J. Biol. Chem. 272, 32436-32442 (1997).

51. Fort, D. G., Herr, T. M., Shaw, P. L., Gutzman, K. E. \& Starren, J. B. Mapping the evolving definitions of translational research. J. Clin. Transl. Sci. 1, 60-66 (2017).

52. Zoellner, J. Nutrition in the Prevention and Treatment of Disease / ScienceDirect. https://www.sciencedirect.com/book/9780128029282/nutrition-in-the-prevention-and-treatmentof-disease (2017).

53. NCATS National Center for Advancing Translational Science. Translational Science Spectrum. National Center for Advancing Translational Sciences https://ncats.nih.gov/translation/spectrum (2015). 


\section{APPENDIX A}

\section{Clinical Translational Science Definition and Purpose}

The definition and purpose of Clinical Translational Science is still evolving. As any new science field, the parameters are set and then adjusted as the field matures. There are five phases of translational research defined by the clinical and translational scientific community (T0-T4). ${ }^{51}$ Translational research is a multi-directional process which integrates multidisciplined science across populations, basic science, policy, and clinical application. ${ }^{52}$ The National Center for Advancing Translational Science speaks of the developing nature of clinical translational science. "At all stages of the spectrum, NCATS develops new approaches, demonstrates their usefulness and disseminates the findings. Patient involvement is a critical feature of all stages in translation.. ${ }^{53}$ The long-term goal of this project is to advance clinical and translational science by demonstrating electrophysiology utilizes a language of physics which speaks fluently from the microscopic to the macroscopic, reaching from the atomic to the cosmic to address human questions in basic science, and clinical medicine.. The initial discussion and first publication address the complexity of the problem translational science faces and the opportunity electrophysiology creates to generate mechanisms for evaluation. The broad scale is then narrowed to illustrate this principle through the second paper which encompasses the basic science research, pre-clinical research, and the clinical research by cell line in-vitro work, xenograft mouse model work, and human resected cancer tumor work respectively; this gives evidence for the functional role of BK (big conductance potassium) channels for targeted treatment in triple negative breast cancer (TNBC). The third discussion continues the precedent for research translation from basic science to clinical practice by epidemiology research to evaluate TNBC in our local patient population of West Virginia utilizing the West Virginia Cancer Registry. Finally, the conclusion outlines the future research which extends beyond this 
reference to generate the collaborative radiation neoadjuvant treatment with targeted ion channels in mice, followed by randomized clinical trials targeting BK ion channels as neoadjuvant treatment with radiation prior to tumor resection in patients with TNBC. In accordance with the goals of the NIH National Center for Advancing Translational Science this research aims to harmonize the diverse measures of scale between molecular biology and clinical medicine. This project completes both T0, T1, and T4 research with defined direction for future research in phases $\mathbf{T} 2$ for clinical application. 


\section{APPENDIX B}

\section{Additional References}

\section{Temporal Lobe Epilepsy, Stroke, and Traumatic Brain Injury: Mechanisms of Hyperpolarized, Depolarized, and Flow-Through Ion Channels Utilized as Tri-Coordinate Biomarkers of Electrophysiologic Dysfunction}

1. Kobow K, Blümcke I. Epigenetics in epilepsy. Neurosci Lett. 2018;667:40-46. [PubMed] [Google Scholar]

2. Karhunen H, Bezvenyuk Z, Nissinen J, Sivenius J, Jolkkonen J, Pitkänen A. Epileptogenesis after cortical photothrombotic brain lesion in rats. Neuroscience. 2007;148:314-324. [PubMed] [Google Scholar]

3. Menon B, Shorvon SD. Ischaemic stroke in adults and epilepsy. Epilepsy

Res. 2009;87:1. [PubMed] [Google Scholar]

4. Khosravani H, Zamponi GW. Voltage-gated calcium channels and idiopathic generalized epilepsies. Physiol Rev. 2006;86:941-966. [PubMed] [Google Scholar]

5. Orsini A, Zara F, Striano P. Recent advances in epilepsy genetics. Neurosci Lett. 2018;667:4-

9. [PubMed] [Google Scholar]

6. Vahedi K, Depienne C, Le FD, Riant F, Chaine P, Trouillard O, et al. Elicited repetitive daily blindness: a new phenotype associated with hemiplegic migraine and SCN1A

mutations. Neurology. 2009;72:1178. [PubMed] [Google Scholar]

7. Pellock JM, Arzimanoglou A, D'Cruz ON, Holmes GL, Nordli D, Shinnar S. Extrapolating evidence of antiepileptic drug efficacy in adults to children $\geq 2$ years of age with focal seizures: The case for disease similarity. Epilepsia. 2017;73:90-94. [PubMed] [Google Scholar]

8. Kucukyuruk B, Richardson RM, Wen HT, Fernandezmiranda JC, R A., Jr Microsurgical anatomy of the temporal lobe and its implications on temporal lobe epilepsy surgery. Epilepsy Res Treat. 2012;2012:769825. [PMC free article] [PubMed] [Google Scholar]

9. Nyquist P, Bautista C, Jichici D, Burns J, Chhangani S, Defilippis M, et al. Prophylaxis of venous thrombosis in neurocritical care patients: An evidence-based guideline: A statement for healthcare professionals from the neurocritical care society. Crit Care Med. 2017;45:476. [PubMed] [Google Scholar]

10. Mckee AC, Daneshvar DH. The neuropathology of traumatic brain injury. Handb Clin Neurol. 2015;127:45-66. [PMC free article] [PubMed] [Google Scholar]

11. Zhang JH. Vascular neural network in subarachnoid hemorrhage. Transl Strok Res. 2014;5:423428. [PMC free article] [PubMed] [Google Scholar]

12. Marhold F, Rosen CL. Novel technique to improve vessel mismatch when using saphenous vein bypass grafts for intracranial revascularization procedures. J Neurosurg. 2010;112:1227-

1231. [PubMed] [Google Scholar] 
13. Turner RC, Dodson SC, Rosen CL, Huber JD. The science of cerebral ischemia and the quest for neuroprotection: navigating past failure to future success. J Neurosurg. 2013;118:1072-1085. [PMC free article] [PubMed] [Google Scholar]

14. Ward NS. Does neuroimaging help to deliver better recovery of movement after stroke? Curr Opin Neurol. 2015;28:323. [PubMed] [Google Scholar]

15. Iyer KK. Effective assessments of electroencephalography during stroke recovery: contemporary approaches and considerations. J Neurophysiol. 2017;118:2521-2525. [PMC free article] [PubMed] [Google Scholar]

16. Pivonkova $\mathrm{H}$, Anderova $\mathrm{M}$. Altered homeostatic functions in reactive astrocytes and their potential as a therapeutic target after brain ischemic injury. Curr Pharm Des. 2017;23:50565074. [PubMed] [Google Scholar]

17. Troakes C, Smyth R, Noor F, Maekawa S, Killick R, King A, et al. Clusterin expression is upregulated following acute head injury and localizes to astrocytes in old head injury. Neuropathology. 2016;37:1224. [PubMed] [Google Scholar]

18. Albrecht J, Zielioska M. Mechanisms of Excessive Extracellular Glutamate Accumulation in Temporal Lobe Epilepsy. Neurochem Res. 2016;42:1724-1734. [PubMed] [Google Scholar]

19. Cembrowski MS, Spruston N. Integrating Results across Methodologies Is Essential for Producing Robust Neuronal Taxonomies. Neuron. 2017;94:747. [PubMed] [Google Scholar]

20. Quintana P, Soto D, Poirot O, Zonouzi M, Kellenberger S, Muller D, et al. Acid-sensing ion channel 1a drives AMPA receptor plasticity following ischaemia and acidosis in hippocampal CA1 neurons. J Physiol. 2015;593:4373-4386. [PMC free article] [PubMed] [Google Scholar]

21. Spruston N. Pyramidal neurons: dendritic structure and synaptic integration. Nat Rev Neurosci. 2008;9:206. [PubMed] [Google Scholar]

22. Bhuiyan MI, Song S, Yuan H, Begum G, Kofler J, Kahle KT, et al. WNK-Cab39-NKCC1 signaling increases the susceptibility to ischemic brain damage in hypertensive rats. J Cereb Blood Flow Metab. 2016;37:2780-2794. [PMC free article] [PubMed] [Google Scholar]

23. Muradashvili N, Tyagi SC, Lominadze D. Localization of fibrinogen in the vasculo-astrocyte interface after cortical contusion injury in mice. Brain Sci. 2017;7 [PMC free article] [PubMed] [Google Scholar]

24. Brennan GP, Henshall DC. microRNAs in the pathophysiology of epilepsy. Neurosci Lett. 2018;667:47-52. [PubMed] [Google Scholar]

25. Weiss N, Miller F, Cazaubon S, Couraud PO. The blood-brain barrier in brain homeostasis and neurological diseases. Biochim Biophys Acta. 2009;1788:842-857. [PubMed] [Google Scholar]

26. Bukeirat M, Sarkar SN, Hu H, Quintana DD, Simpkins JW, Ren X. MiR-34a regulates blood-brain barrier permeability and mitochondrial function by targeting cytochrome c. J Cereb Blood Flow Metab. 2016;36:387. [PMC free article] [PubMed] [Google Scholar] 
27. Hasbargen T, Ahmed MM, Miranpuri G, Li L, Kahle KT, Resnick D, et al. Role of NKCC1 and KCC2 in the development of chronic neuropathic pain following spinal cord injury. Anna N Y Acad Sci. 2010;1198:168-172. [PubMed] [Google Scholar]

28. Lee KPK, Chen J, MacKinnon R. Molecular structure of human KATP in complex with ATP and ADP. Elife. 2017;6 [PMC free article] [PubMed] [Google Scholar]

29. O'Connor ER, Sontheimer H, Spencer DD, de Lanerolle NC. Astrocytes from human hippocampal epileptogenic foci exhibit action potential-like responses. Epilepsia. 1998;39:347. [PubMed] [Google Scholar]

30. Jaggi AS, Kaur A, Bali A, Singh N. Expanding spectrum of sodium potassium chloride co-transporters in the pathophysiology of diseases. Curr Neuropharmacol. 2015;13:369-388. [PMC free article] [PubMed] [Google Scholar]

31. Cong D, Zhu W, Kuo JS, Hu S, Sun D. Ion transporters in brain tumors. Curr Med Chem. 2015;22:1171-1181. [PMC free article] [PubMed] [Google Scholar]

32. Adragna NC, Ravilla NB, Lauf PK, Begum G, Khanna AR, Sun D, et al. Regulated phosphorylation of the $\mathrm{K}-\mathrm{Cl}$ cotransporter $\mathrm{KCC} 3$ is a molecular switch of intracellular potassium content and cell volume homeostasis. Front Cell Neurosci. 2015;9:255. [PMC free article] [PubMed] [Google Scholar]

33. Biel M, Wahlschott C, Michalakis S, Zong X. Hyperpolarization-activated cation channels: From genes to function. Physiol Rev. 2009;89:847-885. [PubMed] [Google Scholar]

34. Benari Y, Khalilov I, Kahle KT, Cherubini E. The GABA excitatory/inhibitory shift in brain maturation and neurological disorders. Neuroscientist. 2012;18:467-486. [PubMed] [Google Scholar]

35. Brennan GP, Baram TZ, Poolos NP. Hyperpolarization-Activated cyclic nucleotide-gated (HCN) channels in epilepsy. Cold Spring Harb Perspect Med. 2016;6:a022384. [PMC free article] [PubMed] [Google Scholar]

36. DiFrancesco JC, DiFrancesco D. Dysfunctional HCN ion channels in neurological diseases. Front Cell Neurosci. 2015;6:174. [PMC free article] [PubMed] [Google Scholar]

37. Lee $\mathrm{CH}$, Mackinnon R. Structures of the human HCN1 hyperpolarization-activated channel. Cell. 2017;168:111-120. [PMC free article] [PubMed] [Google Scholar]

38. Luckewold BP, Turner RC, Logsdon AF, Bailes JE, Huber JD, Rosen CL. Linking traumatic brain injury to chronic traumatic encephalopathy: identification of potential mechanisms leading to neurofibrillary tangle development. J Neurotrauma. 2014;31:1129-1138. [PMC free article] [PubMed] [Google Scholar]

39. Weerasinghe D, Menon P, Vucic S. Hyperpolarization-activated cyclic-nucleotide-gated channels potentially modulate axonal excitability at different thresholds. J Neurophysiol. 2017;118:30443050. [PMC free article] [PubMed] [Google Scholar]

40. Turlova E, Feng ZP, Sun HS. The role of TRPM2 channels in neurons, glial cells and the blood-brain barrier in cerebral ischemia and hypoxia. Acta Pharmacol Sin. 2018;39:713-721. [PMC free article] [PubMed] [Google Scholar] 
41. Mannix R, Berkner J, Mei Z, Alcon S, Hashim J, Robinson S, et al. Adolescent mice demonstrate a distinct pattern of injury after repetitive mild traumatic brain injury. J Neurotrauma. 2017;34:495504. [PMC free article] [PubMed] [Google Scholar]

42. Faria LC, Gu F, Parada I, Barres B, Luo ZD, Prince DA. Epileptiform activity and behavioral arrests in mice overexpressing the calcium channel subunit alpha2delta-1. Neurobiol Dis. 2017;102:7080. [PubMed] [Google Scholar]

43. Calejo Al, Reverendo M, Silva VS, Pereira PM, Santos MA, Zorec R, et al. Differences in the expression pattern of $\mathrm{HCN}$ isoforms among mammalian tissues: sources and implications. Mol Biol Rep. 2014;41:297-307. [PubMed] [Google Scholar]

44. Santina LD, Piano I, Cangiano L, Caputo A, Ludwig A, Cervetto L, et al. Processing of retinal signals in normal and HCN deficient mice. PloS One. 2012;7:e29812. [PMC free article] [PubMed] [Google Scholar]

45. Kahle KT, Merner ND, Friedel P, Silayeva L, Liang B, Khanna A, et al. Genetically encoded impairment of neuronal KCC2 cotransporter function in human idiopathic generalized epilepsy. EMBO Rep. 2014;15:766-774. [PMC free article] [PubMed] [Google Scholar]

46. Merner ND, Chandler MR, Bourassa C, Liang B, Khanna AR, Dion P, et al. Regulatory domain or CpG site variation in SLC12A5, encoding the chloride transporter KCC2, in human autism and schizophrenia. Front Cell Neurosci. 2015;9:386. [PMC free article] [PubMed] [Google Scholar]

47. PhD KTKM, Kevin Staley MD. Altered Neuronal Chloride Homeostasis and Excitatory GABAergic Signaling in Human Temporal Lobe Epilepsy. Epilepsy Curr. 2008;8:51-53. [PMC free article] [PubMed] [Google Scholar]

48. Saitsu H, Watanabe M, Akita T, Ohba C, Sugai K, Ong WP, et al. Impaired neuronal KCC2 function by biallelic SLC12A5 mutations in migrating focal seizures and severe developmental delay. Sci Rep. 2016;6:30072. [PMC free article] [PubMed] [Google Scholar]

49. Frederikse PH, Kasinathan C. KCC2 expression supersedes NKCC1 in mature fiber cells in mouse and rabbit lenses. Mol Vis. 2015;21:1142-1150. [PMC free article] [PubMed] [Google Scholar]

50. Tsukahara T, Masuhara M, Iwai H, Sonomura T, Sato T. Repeated stress-induced expression pattern alterations of the hippocampal chloride transporters KCC2 and NKCC1 associated with behavioral abnormalities in female mice. Biochem Biophys Res Commun. 2015;465:145-151. [PubMed] [Google Scholar]

51. Li X, Zhou J, Chen Z, Chen S, Zhu F, Zhou L. Long-term expressional changes of Na+ -K+ -Cl- cotransporter 1 (NKCC1) and $\mathrm{K}+-\mathrm{Cl}$ - co-transporter 2 (KCC2) in CA1 region of hippocampus following lithium-pilocarpine induced status epilepticus (PISE) Brain Res. 2008;1221:141-146. [PubMed] [Google Scholar]

52. Gagnon KB, Delpire E. Physiology of SLC12 transporters: lessons from inherited human genetic mutations and genetically engineered mouse knockouts. Am J Physiol Cell Physiol. 2013;304:C693714. [PMC free article] [PubMed] [Google Scholar] 
53. Tian Y, Guo SX, Li JR, Du HG, Wang CH, Zhang JM, et al. Topiramate attenuates early brain injury following subarachnoid haemorrhage in rats via duplex protection against inflammation and neuronal cell death. Brain Res. 2015;1622:174. [PubMed] [Google Scholar]

54. Zamponi GW, Striessnig J, Koschak A, Dolphin AC. The physiology, pathology, and pharmacology of voltage-gated calcium channels and their future therapeutic potential. Pharmacol

Rev. 2015;67:821. [PMC free article] [PubMed] [Google Scholar]

55. Hennessey JA, Boczek NJ, Jiang YH, Miller JD, Patrick W, Pfeiffer R, et al. A CACNA1C variant associated with reduced voltage-dependent inactivation, increased CaV1.2 channel window current, and arrhythmogenesis. PloS One. 2014;9:e106982. [PMC free article] [PubMed] [Google Scholar]

56. Nietorostro M, Sandhu G, Bauer CS, Jiruska P, Jefferys JGR, Dolphin AC. Altered expression of the voltage-gated calcium channel subunit $\alpha 2 \delta-1$ : A comparison between two experimental models of epilepsy and a sensory nerve ligation model of neuropathic pain. Neuroscience. 2014;283:124137. [PMC free article] [PubMed] [Google Scholar]

57. Pirone A, Kurt S, Zuccotti A, Rüttiger L, Pilz P, Brown DH, et al. $\alpha 2 \delta 3$ is essential for normal structure and function of auditory nerve synapses and is a novel candidate for auditory processing disorders. J Neurosci. 2014;34:434. [PMC free article] [PubMed] [Google Scholar]

58. Tuluc P, Molenda N, Schlick B, Obermair GJ, Flucher BE, Jurkatrott K. A CaV1.1 Ca2+ channel splice variant with high conductance and voltage-sensitivity alters EC coupling in developing skeletal muscle. Biophys J. 2009;96:35-44. [PMC free article] [PubMed] [Google Scholar]

59. McGonigle $P$, Ruggeri B. Animal models of human disease: challenges in enabling translation. Biochem Pharmacol. 2014;87:162-171. [PubMed] [Google Scholar]

60. Stafstrom CE, Carmant L. Seizures and epilepsy: an overview for neuroscientists. Cold Spring Harb Perspect Med. 2015;5:a022426-a022426. [PMC free article] [PubMed] [Google Scholar]

61. Aghagolzadeh M, Hochberg LR, Cash SS, Truccolo W, Aghagolzadeh M, Hochberg LR, et al. Predicting seizures from local field potentials recorded via intracortical microelectrode arrays. Conf Proc IEEE Eng Med Biol Soc. 2016:6353-6356. [PMC free article] [PubMed] [Google Scholar]

62. Patsalos PN, Zugman M, Lake C, James A, Ratnaraj N, Sander JW. Serum protein binding of 25 antiepileptic drugs in a routine clinical setting: $A$ comparison of free non-protein-bound concentrations. Epilepsia. 2017;58:1234-1243. [PubMed] [Google Scholar]

63. Wolfart J, Laker D. Homeostasis or channelopathy? Acquired cell type-specific ion channel changes in temporal lobe epilepsy and their antiepileptic potential. Front Physiol. 2015;6:168. [PMC free article] [PubMed] [Google Scholar]

64. Landis SC, Amara SG, Asadullah K, Austin CP, Blumenstein R, Bradley EW, et al. A call for transparent reporting to optimize the predictive value of preclinical research. Nature. 2012;490:187-191. [PMC free article] [PubMed] [Google Scholar] 
Opening Large-Conductance Potassium Channels Selectively Induced Cell Death of TripleNegative Breast Cancer

1. Cardiff RD, Kenney N: A compendium of the mouse mammary tumor biologist: from the initial observations in the house mouse to the development of genetically engineered mice. Cold Spring Harb Perspect Biol 2011, 3(6).

2. Perou CM, Sorlie T, Eisen MB, van de Rijn M, Jeffrey SS, Rees CA, Pollack JR, Ross DT, Johnsen $H$, Akslen LA et al: Molecular portraits of human breast tumours. Nature 2000, 406(6797):747752.

3. Kalimutho M, Parsons K, Mittal D, Lopez JA, Srihari S, Khanna KK: Targeted Therapies for TripleNegative Breast Cancer: Combating a Stubborn Disease. Trends Pharmacol Sci 2015, 36(12):822-846.

4. Lehmann BD, Bauer JA, Chen X, Sanders ME, Chakravarthy AB, Shyr Y, Pietenpol JA: Identification of human triple-negative breast cancer subtypes and preclinical models for selection of targeted therapies. J Clin Invest 2011, 121(7):2750-2767.

5. Lehmann BD, Jovanovic B, Chen X, Estrada MV, Johnson KN, Shyr Y, Moses HL, Sanders ME, Pietenpol JA: Refinement of Triple-Negative Breast Cancer Molecular Subtypes: Implications for Neoadjuvant Chemotherapy Selection. PLoS One 2016, 11(6):e0157368.

6. Ryden L, Jirstrom K, Haglund M, Stal O, Ferno M: Epidermal growth factor receptor and vascular endothelial growth factor receptor $\mathbf{2}$ are specific biomarkers in triple-negative breast cancer. Results from a controlled randomized trial with long-term follow-up. Breast cancer research and treatment 2010, 120(2):491-498.

7. Fedele $\mathrm{P}$, Orlando L, Cinieri S: Targeting triple negative breast cancer with histone deacetylase inhibitors. Expert Opin Investig Drugs 2017, 26(11):1199-1206.

8. Abdul Kadir L, Stacey M, Barrett-Jolley R: Emerging Roles of the Membrane Potential: Action Beyond the Action Potential. Frontiers in Physiology 2018, 9(1661).

9. Urrego D, Tomczak AP, Zahed F, Stühmer W, Pardo LA: Potassium channels in cell cycle and cell proliferation. Philos Trans R Soc Lond B Biol Sci 2014, 369(1638):20130094-20130094.

10. Blackiston DJ, McLaughlin KA, Levin M: Bioelectric controls of cell proliferation: ion channels, membrane voltage and the cell cycle. Cell Cycle 2009, 8(21):3519-3528.

11. Marino AA, Iliev IG, Schwalke MA, Gonzalez E, Marler KC, Flanagan CA: Association between cell membrane potential and breast cancer. Tumour Biol 1994, 15(2):82-89.

12. Berzingi S, Newman M, Yu H-G: Altering bioelectricity on inhibition of human breast cancer cells. Cancer Cell International 2016, 16(1):72.

13. Contreras GF, Castillo K, Enrique N, Carrasquel-Ursulaez W, Castillo JP, Milesi V, Neely A, Alvarez O, Ferreira G, Gonzalez C et al: A BK (Slo1) channel journey from molecule to physiology. Channels (Austin) 2013, 7(6):442-458.

14. Yang H, Zhang G, Cui J: BK channels: multiple sensors, one activation gate. Frontiers in Physiology 2015, 6(29).

15. Khaitan D, Sankpal UT, Weksler B, Meister EA, Romero IA, Couraud PO, Ningaraj NS: Role of KCNMA1 gene in breast cancer invasion and metastasis to brain. BMC Cancer 2009, 9:258.

16. Oeggerli M, Tian Y, Ruiz C, Wijker B, Sauter G, Obermann E, Güth U, Zlobec I, Sausbier M, Kunzelmann K et al: Role of KCNMA1 in Breast Cancer. PLoS One 2012, 7(8):e41664. 
17. Ma YG, Liu WC, Dong S, Du C, Wang XJ, Li JS, Xie XP, Wu L, Ma DC, Yu ZB et al: Activation of $\mathrm{BK}(\mathrm{Ca})$ channels in zoledronic acid-induced apoptosis of MDA-MB-231 breast cancer cells. PLOS One 2012, 7(5):e37451.

18. Weinstein JN, Collisson EA, Mills GB, Shaw KR, Ozenberger BA, Ellrott K, Shmulevich I, Sander C, Stuart JM: The Cancer Genome Atlas Pan-Cancer analysis project. Nat Genet 2013, 45(10):11131120.

19. Cerami E, Gao J, Dogrusoz U, Gross BE, Sumer SO, Aksoy BA, Jacobsen A, Byrne CJ, Heuer ML, Larsson $\mathrm{E}$ et al: The $\mathrm{cBio}$ cancer genomics portal: an open platform for exploring multidimensional cancer genomics data. Cancer Discov 2012, 2(5):401-404.

20. Trapnell C, Williams BA, Pertea G, Mortazavi A, Kwan G, van Baren MJ, Salzberg SL, Wold BJ, Pachter L: Transcript assembly and quantification by RNA-Seq reveals unannotated transcripts and isoform switching during cell differentiation. Nat Biotech 2010, 28(5):511-515.

21. Yu H-G, McLaughlin S, Newman M, Brundage K, Ammer A, Martin K, Coad J: Altering calcium influx for selective destruction of breast tumor. BMC Cancer 2017, 17(1):169.

22. Huang J, Lin YC, Hileman S, Martin KH, Hull R, Yu HG: PP2 Prevents beta-Adrenergic Stimulation of Cardiac Pacemaker Activity. J Cardiovasc Pharmacol 2014, 63(6):533-543.

23. Ciriello G, Gatza ML, Beck AH, Wilkerson MD, Rhie SK, Pastore A, Zhang H, McLellan M, Yau C, Kandoth $\mathrm{C}$ et al: Comprehensive Molecular Portraits of Invasive Lobular Breast Cancer. Cell 2015, 163(2):506-519.

24. Edfors F, Danielsson F, Hallstrom BM, Kall L, Lundberg E, Ponten F, Forsstrom B, Uhlen M: Genespecific correlation of RNA and protein levels in human cells and tissues. Mol Syst Biol 2016, 12(10):883.

25. Sailer CA, Kaufmann WA, Kogler M, Chen L, Sausbier U, Ottersen OP, Ruth P, Shipston MJ, Knaus HG: Immunolocalization of BK channels in hippocampal pyramidal neurons. The European journal of neuroscience 2006, 24(2):442-454.

26. Sausbier U, Sausbier M, Sailer CA, Arntz C, Knaus HG, Neuhuber W, Ruth P: Ca2+-activated K+ channels of the BK-type in the mouse brain. Histochemistry and cell biology 2006, 125(6):725741.

27. Knaus H-G, Eberhart A, Koch ROA, Munujos P, Schmalhofer WA, Warmke JW, Kaczorowski GJ, Garcia ML: Characterization of Tissue-expressed $\alpha$ Subunits of the High Conductance Ca2+activated K+ Channel. Journal of Biological Chemistry 1995, 270(38):22434-22439.

28. Galvez A, Gimenez-Gallego G, Reuben JP, Roy-Contancin L, Feigenbaum P, Kaczorowski GJ, Garcia ML: Purification and characterization of a unique, potent, peptidyl probe for the high conductance calcium-activated potassium channel from venom of the scorpion Buthus tamulus. J Biol Chem 1990, 265(19):11083-11090.

29. Sun XP, Schlichter LC, Stanley EF: Single-channel properties of BK-type calcium-activated potassium channels at a cholinergic presynaptic nerve terminal. J Physiol 1999, 518 ( Pt 3):639651.

30. Romine JL, Martin SW, Meanwell NA, Gribkoff VK, Boissard CG, Dworetzky SI, Natale J, Moon S, Ortiz A, Yeleswaram S et al: 3-[(5-Chloro-2-hydroxyphenyl)methyl]-5-[4(trifluoromethyl)phenyl ]-1,3,4-oxadiazol-2(3H)-one, BMS-191011: opener of largeconductance $\mathrm{Ca}(2+)$-activated potassium (maxi-K) channels, identification, solubility, and SAR. J Med Chem 2007, 50(3):528-542.

31. Bentzen BH, Nardi A, Calloe K, Madsen LS, Olesen SP, Grunnet M: The small molecule NS11021 is a potent and specific activator of Ca2+-activated big-conductance $\mathrm{K}+$ channels. Molecular pharmacology 2007, 72(4):1033-1044.

32. Chavez KJ, Garimella SV, Lipkowitz S: Triple negative breast cancer cell lines: one tool in the search for better treatment of triple negative breast cancer. Breast Dis 2010, 32(1-2):35-48. 
33. Chen X, Yan J, Aldrich RW: BK channel opening involves side-chain reorientation of multiple deep-pore residues. Proc Natl Acad Sci U S A 2014, 111(1):E79-88.

34. Maeno E, Ishizaki Y, Kanaseki T, Hazama A, Okada Y: Normotonic cell shrinkage because of disordered volume regulation is an early prerequisite to apoptosis. Proc Natl Acad Sci U S A 2000, 97(17):9487-9492.

35. Galluzzi L, Bravo-San Pedro JM, Vitale I, Aaronson SA, Abrams JM, Adam D, Alnemri ES, Altucci L, Andrews $D$, Annicchiarico-Petruzzelli $M$ et al: Essential versus accessory aspects of cell death: recommendations of the NCCD 2015. Cell death and differentiation 2015, 22(1):58-73.

36. van Zijl F, Krupitza G, Mikulits W: Initial steps of metastasis: Cell invasion and endothelial transmigration. Mutation Research 2011, 728(1-2):23-34.

37. Indelicato M, Pucci B, Schito L, Reali V, Aventaggiato M, Mazzarino MC, Stivala F, Fini M, Russo MA, Tafani M: Role of hypoxia and autophagy in MDA-MB-231 invasiveness. J Cell Physiol 2010, 223(2):359-368.

38. Scott JM, Nilsen TS, Gupta D, Jones LW: Exercise Therapy and Cardiovascular Toxicity in Cancer. Circulation 2018, 137(11):1176-1191.

39. Elmore S: Apoptosis: a review of programmed cell death. Toxicol Pathol 2007, 35(4):495-516.

40. https://www.ncbi.nlm.nih.gov/gene/3778.

41. Mehta LS, Watson KE, Barac A, Beckie TM, Bittner V, Cruz-Flores S, Dent S, Kondapalli L, Ky B, Okwuosa T et al: Cardiovascular Disease and Breast Cancer: Where These Entities Intersect: A Scientific Statement From the American Heart Association. Circulation 2018, 137(8):e30-e66.

42. Prevarskaya N, Skryma R, Shuba Y: Ion channels and the hallmarks of cancer. Trends in Molecular Medicine 2010, 16(3):107-121.

43. Bentzen BH, Olesen SP, Ronn LC, Grunnet M: BK channel activators and their therapeutic perspectives. Front Physiol 2014, 5:389.

44. Humphries ESA, Dart C: Neuronal and Cardiovascular Potassium Channels as Therapeutic Drug Targets: Promise and Pitfalls. J Biomol Screen 2015, 20(9):1055-1073.

45. Roden DM, Balser JR, George AL, Jr., Anderson ME: Cardiac ion channels. Annu Rev Physio/ 2002, 64:431-475.

46. Orrenius S, Zhivotovsky B, Nicotera P: Regulation of cell death: the calcium-apoptosis link. Nat Rev Mol Cell Biol 2003, 4(7):552-565.

47. Schanne FA, Kane AB, Young EE, Farber JL: Calcium dependence of toxic cell death: a final common pathway. Science 1979, 206(4419):700-702.

48. Taylor JT, Huang L, Pottle JE, Liu K, Yang Y, Zeng X, Keyser BM, Agrawal KC, Hansen JB, Li M: Selective blockade of T-type $\mathrm{Ca2}+$ channels suppresses human breast cancer cell proliferation. Cancer Lett 2008, 267(1):116-124.

49. Pera E, Kaemmerer E, Milevskiy MJ, Yapa KT, O'Donnell JS, Brown MA, Simpson F, Peters AA, Roberts-Thomson SJ, Monteith GR: The voltage gated $\mathrm{Ca}(2+)$-channel Cav3.2 and therapeutic responses in breast cancer. Cancer Cell Int 2016, 16.

50. Taylor JT, Zeng XB, Pottle JE, Lee K, Wang AR, Yi SG, Scruggs JA, Sikka SS, Li M: Calcium signaling and T-type calcium channels in cancer cell cycling. World J Gastroenterol 2008, 14(32):49844991.

51. Huang X, Jan LY: Targeting potassium channels in cancer. J Cell Biol 2014, 206(2):151-162.

52. Restrepo-Angulo I, Bañuelos C, Camacho J: Ion Channel Regulation by Sex Steroid Hormones and Vitamin D in Cancer: A Potential Opportunity for Cancer Diagnosis and Therapy. Frontiers in Pharmacology 2020, 11(152).

53. Dixon JE, McKinnon D: Quantitative analysis of potassium channel mRNA expression in atrial and ventricular muscle of rats. Circ Res 1994, 75(2):252-260. 
54. Teisseyre A, Palko-Labuz A, Sroda-Pomianek K, Michalak K: Voltage-Gated Potassium Channel Kv1.3 as a Target in Therapy of Cancer. Frontiers in Oncology 2019, 9(933).

\section{Triple Negative Breast Cancer: Exponential Tumor Grade Increase with Age of Diagnosis}

1. Shah, R., Rosso, K. \& Nathanson, S. D. Pathogenesis, prevention, diagnosis and treatment of breast cancer. World J. Clin. Oncol. 5, 283-298 (2014).

2. Alkabban, F. M. \& Ferguson, T. Cancer, Breast. in StatPearls (StatPearls Publishing, 2019).

3. Greenup, R. et al. Prevalence of BRCA mutations among women with triple-negative breast cancer (TNBC) in a genetic counseling cohort. Ann. Surg. Oncol. 20, 3254-3258 (2013).

4. Breast Cancer.Org. Triple-Negative Breast Cancer: Overview, Treatment, and More. Breastcancer.org https://www.breastcancer.org/symptoms/diagnosis/trip_neg (2019).

5. Lehmann, B. D. et al. Identification of human triple-negative breast cancer subtypes and preclinical models for selection of targeted therapies. J. Clin. Invest. 121, 2750-2767 (2011).

6. Dietze, E. C., Sistrunk, C., Miranda-Carboni, G., O’Regan, R. \& Seewaldt, V. L. Triplenegative breast cancer in African-American women: disparities versus biology. Nat. Rev. Cancer 15, 248-254 (2015).

7. Lara-Medina, F. et al. Triple-negative breast cancer in Hispanic patients: high prevalence, poor prognosis, and association with menopausal status, body mass index, and parity. Cancer 117, 3658-3669 (2011).

8. Vona-Davis, L. et al. Triple-Negative Breast Cancer and Obesity in a Rural Appalachian Population. Cancer Epidemiol. Prev. Biomark. 17, 3319-3324 (2008).

9. Plasilova, M. L. et al. Features of triple-negative breast cancer. Medicine (Baltimore) 95, (2016). 
10. Ademuyiwa, F. O., Tao, Y., Luo, J., Weilbaecher, K. \& Ma, C. X. Differences in the mutational landscape of triple-negative breast cancer in African Americans and Caucasians. Breast Cancer Res. Treat. 161, 491-499 (2017).

11. Anderson, K. et al. Family history of breast and ovarian cancer and triple negative subtype in hispanic/latina women. SpringerPlus 3, 727 (2014).

12. Doepker, M. P., Holt, S. D., Durkin, M. W., Chu, C. H. \& Nottingham, J. M. Triple-Negative Breast Cancer: A Comparison of Race and Survival. Am. Surg. 84, 881-888 (2018).

13. Nahleh, Z. et al. Disparities in breast cancer: a multi-institutional comparative analysis focusing on American Hispanics. Cancer Med. 7, 2710-2717 (2018).

14. Newman, L. A. \& Kaljee, L. M. Health Disparities and Triple-Negative Breast Cancer in African American Women: A Review. JAMA Surg. 152, 485-493 (2017).

15. Dietze, E. C., Chavez, T. A. \& Seewaldt, V. L. Obesity and Triple-Negative Breast Cancer: Disparities, Controversies, and Biology. Am. J. Pathol. 188, 280-290 (2018).

16. Siddharth, S. \& Sharma, D. Racial Disparity and Triple-Negative Breast Cancer in AfricanAmerican Women: A Multifaceted Affair between Obesity, Biology, and Socioeconomic Determinants. Cancers 10, (2018).

17. Abraham, J. et al. Triple-negative breast cancer in West Virginia. W. V. Med. J. 105 Spec No, 54-59 (2009).

18. Centers for Disease Control and Prevention. USCS Data Visualizations. Leading cancer cases and deaths, female, 2016.2019 https://gis.cdc.gov/grasp/USCS/DataViz.html.

19. Census, U.S. U.S. Census Bureau QuickFacts: West Virginia; United States. https://www.census.gov/quickfacts/fact/table/WV,US/RHI125218\#RHI125218. 
20. Bureau, U. C. State Rankings. The United States Census Bureau. 2012 statistical abstract; state rankings https://www.census.gov/library/publications/2011/compendia/statab/131ed/rankings.html (2019).

21. Centers for Disease Control and Prevention. DNPAO Data, Trends and Maps: Explore by Topic | CDC.

https://nccd.cdc.gov/dnpao_dtm/rdPage.aspx?rdReport=DNPAO_DTM.ExploreByTopic\&isl Class=OWS\&islTopic $=$ OWS1\&go=GO. (2019).

22. Bureau, U. C. State-to-State Migration Flows. The United States Census Bureau https://www.census.gov/data/tables/time-series/demo/geographic-mobility/state-to-statemigration.html.

23. WV OEPS. WV OEPS West Virginia Department of Health and Human Resources, Cancer Registry. https://oeps.wv.gov/cancer/Pages/default.aspx (2019).

24. SEER Registrars. Reporting Guidelines - SEER Registrars. SEER https://seer.cancer.gov/registrars/guidelines.html (2019).

25. Efron, B. The Efficiency of Logistic Regression Compared to Normal Discriminant Analysis. J. Am. Stat. Assoc. 70, 892-898 (1975).

26. Anders, C. K. \& Carey, L. A. Biology, Metastatic Patterns, and Treatment of Patients with Triple-Negative Breast Cancer. Clin. Breast Cancer 9, S73-S81 (2009).

27. Zhang, Z. Model building strategy for logistic regression: purposeful selection. Ann. Transl. Med. 4, (2016).

28. Lehmann, B. D. et al. Refinement of Triple-Negative Breast Cancer Molecular Subtypes: Implications for Neoadjuvant Chemotherapy Selection. PLOS ONE 11, e0157368 (2016). 
29. Cynthia X. Ma, M. D. The Cancer Genome Atlas: Clinical Applications for Breast Cancer. Cancer Network https://www.cancernetwork.com/review-article/cancer-genome-atlas-clinicalapplications-breast-cancer (2013).

30. Palmieri, D. et al. Analyses of Resected Human Brain Metastases of Breast Cancer Reveal the Association between Up-Regulation of Hexokinase 2 and Poor Prognosis. Mol. Cancer Res. 7, 1438-1445 (2009).

31. Vieira, A. F. \& Schmitt, F. An Update on Breast Cancer Multigene Prognostic TestsEmergent Clinical Biomarkers. Front. Med. 5, (2018). 